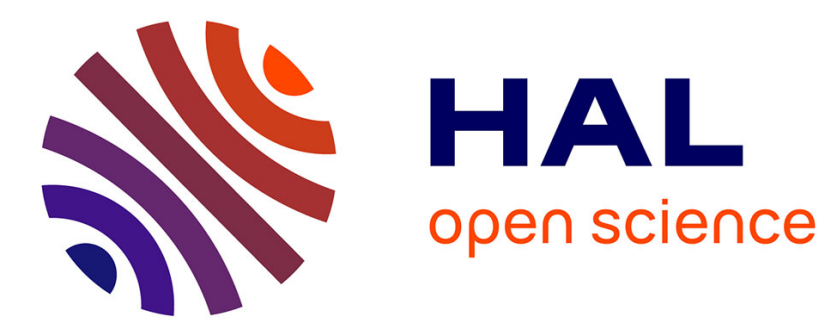

\title{
On the equivariant blow-Nash classification of simple invariant Nash germs \\ Fabien Priziac
}

\section{To cite this version:}

Fabien Priziac. On the equivariant blow-Nash classification of simple invariant Nash germs. Bulletin de la société mathématique de France, 2020, 10.24033/bsmf.2808 . hal-01324842

\section{HAL Id: hal-01324842 \\ https://hal.science/hal-01324842}

Submitted on 1 Jun 2016

HAL is a multi-disciplinary open access archive for the deposit and dissemination of scientific research documents, whether they are published or not. The documents may come from teaching and research institutions in France or abroad, or from public or private research centers.
L'archive ouverte pluridisciplinaire HAL, est destinée au dépôt et à la diffusion de documents scientifiques de niveau recherche, publiés ou non, émanant des établissements d'enseignement et de recherche français ou étrangers, des laboratoires publics ou privés. 


\title{
On the equivariant blow-Nash classification of simple invariant Nash germs
}

\author{
Fabien Priziac
}

\begin{abstract}
We make progress towards the classification of simple Nash germs invariant under the involution changing the sign of the first coordinate, with respect to equivariant blow-Nash equivalence, which is an equivariant Nash version of blow-analytic equivalence, taking advantage of invariants for this relation, the equivariant zeta functions.
\end{abstract}

\section{Introduction}

The classification of real analytic germs requires to choose carefully the used equivalence relation. One may think about the (right) $C^{1}$-equivalence. However, it is too strong, as illustrated by the example of the Whitney family $f_{t}(x, y)=x y(y-x)(y-t x), t>1\left(f_{t}\right.$ and $f_{t^{\prime}}$ are $C^{1}$-equivalent if and only if $t=t^{\prime}$ ), while the topological equivalence is too rough. In [18], T.-C. Kuo suggested an equivalence relation for which Whitney family has only one equivalence class : the blow-analytic equivalence. More generally, any analytically parametrized family of isolated singularities has a locally finite classification with respect to blow-analytic equivalence.

Two real analytic germs are said blow-analytically equivalent if, roughly speaking, they become analytically equivalent after compositions with real modifications, e.g. compositions of blowings-up along smooth centers. From the definition of this equivalence relation, further studies on real analytic germs were stimulated. In particular, invariants have been constructed for blow-analytic equivalence, like the Fukui invariants ([15]) as well as the zeta functions of S. Koike and A. Parusiński ([17]), inspired by the motivic zeta functions of J. Denef and F. Loeser $([8])$, using the Euler characteristic with compact supports as a motivic measure.

A refinement of blow-analytic equivalence has been defined for Nash germs, that is germs of real analytic functions with a semialgebraic graph, by G. Fichou in [10] : the blow-Nash equivalence, that is Nash equivalence after compositions with Nash modifications. The involved algebraicity allowed him to use the virtual Poincaré polynomial ([21] and [9]), which is an additive and multiplicative invariant on $\mathcal{A S}$ sets ([19] and [20]) encoding more information than the Euler characteristic with compact supports, in order to define new zeta functions,

Keywords : simple invariant Nash germs, equivariant blow-Nash equivalence, $A B C D E F$-singularities, equivariant zeta functions, equivariant virtual Poincaré series.

2010 Mathematics Subject Classification : 14B05, 14P20, 14P25, 32S15, 57S17, 57S25.

Research partially supported by a Japan Society for the Promotion of Science (JSPS) Postdoctoral Fellowship. 
invariant for the blow-Nash equivalence of Nash germs. Recently, J.-B. Campesato gave in [6] an equivalent alternative definition of blow-Nash equivalence as arc-analytic equivalence, proving that the blow-Nash equivalence of [10] was indeed an equivalence relation, and defined a more general invariant for it, the motivic local zeta function.

In [13], G. Fichou used his zeta functions of [10] to classify the simple Nash germs (a germ is called simple if sufficiently small perturbations provide only finitely many analytic classes) with respect to blow-Nash equivalence. He showed that this classification actually coincides with the real analytic one, that is the $A D E$-classification of [2]. An analog result for blow-analytic equivalence is not known.

In this paper, we are interested in real analytic germs invariant under right composition with the action of the group $G=\mathbb{Z} / 2 \mathbb{Z}$ only changing the sign of the first coordinate (that we will simply call invariant germs). In [23], we defined the equivariant blow-Nash equivalence for invariant Nash germs, which is, roughly speaking, an equivariant Nash equivalence after compositions with equivariant Nash modifications. Using the equivariant virtual Poincaré series ([14]), which is an additive invariant on $G-\mathcal{A S}$ sets, as a motivic measure, we constructed "equivariant" zeta functions which are invariants for the equivariant blow-Nash equivalence.

Similarly to the non-equivariant frame, we ask if the equivariant blow-Nash classification of invariant Nash germs could coincide with the equivariant Nash classification for sufficiently "tame" invariant singularities. The equivariant analytic classification of simple invariant real analytic germs has been established by V. I. Arnold in [1] and recalled in [16] by V. V. Goryunov. The representatives for this classification are the invariant singularities $A_{k}, B_{k}, C_{k}, D_{k}, E_{6}$, $E_{7}, E_{8}$ and $F_{4}$ (see theorem 2.1 below). We will first show that a simple invariant Nash germ is $G$-blow-Nash equivalent (and even $G$-Nash equivalent) to one of these germs. The largest part of our study will then consist in trying to distinguish, with respect to $G$-blow-Nash equivalence, the invariant $A B C D E F$-singularities, using notably the equivariant zeta functions.

For some cases, we will be faced with either the equality of the respective equivariant zeta functions of a couple of germs or the fact that they are equal if and only if the respective equivariant virtual Poincaré series of specific sets are equal. The former situation is in particular due to the fact that the equivariant virtual Poincaré series can not distinguish two different algebraic actions on a same sphere as soon as there is at least one fixed point. As for the latter situation, we do not know if the invariance of the virtual Poincaré polynomial under bijection with $\mathcal{A S}$ graph (see [22]) "generalizes" to an invariance of the equivariant virtual Poincaré series under equivariant bijection with $\mathcal{A S}$ graph. If this was proven to be true, it should allow to compute all the coefficients of the considered equivariant zeta functions.

The next section is devoted to the equivariant Nash classification of simple invariant Nash germs : we prove that it coincides with the equivariant real analytic classification of [1] and [16]. Indeed, two invariant Nash germs are equivariantly Nash equivalent if and only if they are equivariantly analytically equivalent (proposition 2.3). This can be deduced from an equivariant Nash approximation theorem of E. Bierstone and P. Milman in [4].

In section 3, we justify the fact that a germ $G$-Nash equivalent to a germ of the list $A B C D E F$ is in particular $G$-blow-Nash equivalent to it. On the other hand, one can notice that, forgetting the $G$-action, the invariant singularities $A_{k}$ and $B_{k}$, resp. $C_{k}$ and $D_{k}, E_{6}$ and $F_{4}$, are both $A$-, resp. $D$-, $E$-, singularities. Since equivariant blow-Nash equivalence is 
a particular case of blow-Nash equivalence and because the $A D E$-singularities are not blowNash equivalent to one another ([13]), we are reduced to compare, with respect to $G$-blow-Nash equivalence, the invariant germs of the families $A_{k}$ and $B_{k}$, resp. $C_{k}$ and $D_{k}, E_{6}$ and $F_{4}$.

The section 4 recalls the definition of the tools we are going to use to do so : the equivariant zeta functions. Each of the sections 6,7 and 8 is devoted to the comparison of the invariant germs of a specific couple of families $\left(A_{k}\right.$ and $B_{k}, C_{k}$ and $D_{k}$, and finally $E_{6}$ and $\left.F_{4}\right)$. We proceed as follows. We begin by computing the first coefficients of the equivariant zeta functions (that is the coefficients of degree strictly smaller than the degree of the germs) in order to extract first cases of non-G-blow-Nash equivalence. Reducing our study to the remaining cases, we then compute the coefficient of degree equal to the degree of the germs. Finally, for the cases for which it is not sufficient, we compare the last terms of the respective equivariant zeta functions.

These comparisons lead to interesting examples of computations of equivariant virtual Poincaré series. The first one, to which is devoted section 5, is the computation of the equivariant virtual Poincaré series of the fibers over $0,-1$ and +1 of the quadratic forms $Q_{p, q}(y):=\sum_{i=1}^{p} y_{i}^{2}-\sum_{j=1}^{q} y_{p+j}^{2}$, equipped with four different actions of $G$.

Acknowledgements. The author wishes to thank J.-B. Campesato, G. Fichou, T. Fukui, A. Parusiński, G. Rond and M. Shiota for useful discussions and comments.

\section{Equivariant Nash classification of invariant simple Nash germs}

Consider the affine space $\mathbb{R}^{n}$ with coordinates $\left(x_{1}, \ldots, x_{n}\right)$. We denote by $s$ the involution of $\mathbb{R}^{n}$ changing the sign of the first coordinate $x_{1}$ :

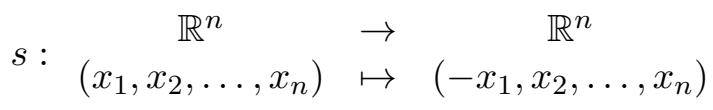

This equips $\mathbb{R}^{n}$ with a linear action of the group $G=\left\{i d_{\mathbb{R}^{n}}, s\right\}$.

In this paper, a function germ $f:\left(\mathbb{R}^{n}, 0\right) \rightarrow(\mathbb{R}, 0)$ will be said invariant if $f$ is invariant under right composition with $s$, that is if $f$ is the germ of an equivariant function (we equip $\mathbb{R}$ with the trivial action of $G$ ).

In [1] and [16] is given the classification of invariant simple real analytic germs $\left(\mathbb{R}^{n}, 0\right) \rightarrow$ $(\mathbb{R}, 0)$ with respect to equivariant analytic equivalence, that is right equivalence via an equivariant analytic diffeomorphism $\left(\mathbb{R}^{n}, 0\right) \rightarrow\left(\mathbb{R}^{n}, 0\right):$

Theorem 2.1 ([1], [16]). An invariant simple real analytic function germ $\left(\mathbb{R}^{n}, 0\right) \rightarrow(\mathbb{R}, 0)$ is equivariantly analytically equivalent to one and only one invariant germ of the following list :

$$
\begin{array}{ll}
A_{k}, k \geq 0: \pm x_{1}^{2} \pm x_{2}^{k+1}+Q, & E_{6}: \pm x_{1}^{2}+x_{2}^{3} \pm x_{3}^{4}+Q, \\
B_{k}, k \geq 2: \pm x_{1}^{2 k} \pm x_{2}^{2}+Q, & E_{7}: \pm x_{1}^{2}+x_{2}^{3}+x_{2} x_{3}^{3}+Q, \\
C_{k}, k \geq 3: x_{1}^{2} x_{2} \pm x_{2}^{k}+Q, & E_{8}: \pm x_{1}^{2}+x_{2}^{3}+x_{3}^{5}+Q, \\
D_{k}, k \geq 4: \pm x_{1}^{2}+x_{2}^{2} x_{3} \pm x_{3}^{k-1}+Q, & F_{4}: \pm x_{1}^{4}+x_{2}^{3}+Q,
\end{array}
$$

where $Q= \pm x_{s}^{2} \pm \cdots \pm x_{n}^{2}$, with $s=4$ for singularities $D_{k}$ and $E_{k}$, and $s=3$ in the other cases. 
Remark 2.2. If we forget the action of the involution $s$ on $\mathbb{R}^{n}$, notice that the families $A_{k}$ and $B_{k}$, resp. $C_{k}$ and $D_{k}, E_{6}$ and $F_{4}, E_{7}, E_{8}$, of Theorem 2.1 are singularities $A$, resp. $D, E_{6}, E_{7}$, $E_{8}$.

In this paper, we are interested in the classification of invariant Nash germs $\left(\mathbb{R}^{n}, 0\right) \rightarrow(\mathbb{R}, 0)$, that is germs of equivariant analytic functions with semialgebraic graph. Recall (see for instance [5] Corollary 8.1.6) that a Nash germ can be considered as an algebraic power series, via its Taylor series. The above classification is also valid for invariant simple Nash germs with respect to equivariant Nash equivalence, that is right equivalence via an equivariant Nash diffeomorphism $\left(\mathbb{R}^{n}, 0\right) \rightarrow\left(\mathbb{R}^{n}, 0\right)$, according to the following proposition :

Proposition 2.3. Let $f, h:\left(\mathbb{R}^{n}, 0\right) \rightarrow(\mathbb{R}, 0)$ be two invariant Nash germs. Then $f$ and $h$ are equivariantly Nash equivalent if and only if they are equivariantly analytically equivalent.

This property is a particular case of the following result :

Theorem 2.4. Let $G$ be a reductive algebraic group acting linearly on $\mathbb{R}^{n}$ and $\mathbb{R}^{p}$. Consider two equivariant Nash germs $f:\left(\mathbb{R}^{n}, 0\right) \rightarrow\left(\mathbb{R}^{p}, 0\right)$ and $h:\left(\mathbb{R}^{n}, 0\right) \rightarrow\left(\mathbb{R}^{p}, 0\right)$. If $f$ and $h$ are equivariantly analytically equivalent, then they are equivariantly Nash equivalent.

Remark 2.5. - Since a Nash diffeomorphism is in particular analytic, the converse is obviously true.

- Any finite group is reductive.

Proof (of Theorem 2.4). Suppose there exists an equivariant analytic diffeomorphism $\phi:\left(\mathbb{R}^{n}, 0\right) \rightarrow$ $\left(\mathbb{R}^{n}, 0\right)$ such that $f \circ \phi=h$. Denote $F(x, y):=f(y)-h(x)$ for $x, y \in \mathbb{R}^{n}$. Then $F:\left(\mathbb{R}^{2 n}, 0\right) \rightarrow$ $\left(\mathbb{R}^{p}, 0\right)$ is a Nash germ and can be considered as an algebraic power series in $\mathbb{R}_{\text {alg }}[[x, y]]^{p}$, and $\phi(x)$ as an equivariant convergent power series in $\mathbb{R}\{x, y\}$ such that $F(x, \phi(x))=0$.

Therefore, by Theorem A of [4] and Example 11.3 of [25], we can approximate $\phi(x)$ by an equivariant algebraic power series $\widetilde{\phi}(x)$ such that $F(x, \widetilde{\phi}(x))=0$, and we do the approximation closely enough so that $\widetilde{\phi}(x)$ remains a diffeomorphism. As a consequence, $\widetilde{\phi}:\left(\mathbb{R}^{n}, 0\right) \rightarrow\left(\mathbb{R}^{n}, 0\right)$ is an equivariant Nash diffeomorphism such that $f \circ \widetilde{\phi}=h$.

Remark 2.6. Actually, Theorem A of [4] is about approximation of equivariant formal solutions of polynomial equations by equivariant algebraic power series but it is also true for algebraic power series equations. Indeed, following G. Rond's ideas, it is possible to reduce to the case of polynomial equations as in [3] Lemma 5.2 and [7] Reduction (2) of the proof of Theorem 1.1, using arguments of the proof of Lemma 8.1 in [24], along with the fact that the morphism $\mathbb{R}[x, y]_{(x, y)} \rightarrow \mathbb{R}_{a l g}[[x, y]]$ is faithfully flat by [5] Corollary 8.7.16.

\section{Equivariant blow-Nash equivalence}

Now, we want to study the classification of invariant simple Nash germs with respect to $G$ blow-Nash equivalence via an equivariant blow-Nash isomorphism : see [23] for the definition of $G$-blow-Nash equivalence via an equivariant blow-Nash isomorphism.

First, we have the following : 
Proposition 3.1. An invariant simple Nash germ $\left(\mathbb{R}^{n}, 0\right) \rightarrow(\mathbb{R}, 0)$ is $G$-blow-Nash equivalent via an equivariant blow-Nash isomorphism to an invariant germ of the list of Theorem 2.1.

Proof. This comes from the fact that if $f$ and $h$ are equivariantly Nash equivalent invariant Nash germs $\left(\mathbb{R}^{n}, 0\right) \rightarrow(\mathbb{R}, 0)$, then they are $G$-blow-Nash equivalent via an equivariant blowNash isomorphism.

Indeed, if $f^{-1}(0)$, resp. $h^{-1}(0)$, has only one irreducible component at $0 \in \mathbb{R}^{n}$, this is straightforward. If not, we perform a composition $\sigma_{f}:\left(M_{f}, \sigma_{f}^{-1}(0)\right) \rightarrow\left(\mathbb{R}^{n}, 0\right)$, resp. $\sigma_{h}$ : $\left(M_{h}, \sigma_{h}^{-1}(0)\right) \rightarrow\left(\mathbb{R}^{n}, 0\right)$, of successive equivariant blowings-up along $G$-invariant smooth Nash centers such that

- the irreducible components of the strict transform of $f^{-1}(0)$ by $\sigma_{f}$, resp. of $h^{-1}(0)$ by $\sigma_{h}$, do not intersect,

- $f \circ \sigma_{f}$ and $j a c \sigma_{f}$, resp. $h \circ \sigma_{h}$ and $j a c \sigma_{h}$, have only normal crossings simultaneously,

- there exists a finite collection of $G$-invariant affine charts for $\sigma_{f}$, resp. for $\sigma_{h}$, such that, on each of these charts, the action of $G$ is of the form

$$
\left(x_{1}, x_{2}, \ldots, x_{n}\right) \mapsto\left(\epsilon_{1} x_{1}, \epsilon_{2} x_{2}, \ldots, \epsilon_{n} x_{n}\right),
$$

where $\epsilon_{i} \in\{ \pm 1\}$ (so that the action of $G$ on $M_{f}$, resp. on $M_{h}$, can be locally linearized on the normal crossings, in the sense of [23]),

- after each blowing-up, $f$ and $h$ remain equivariantly Nash equivalent.

The second step will then consist in understanding the relations, with respect to $G$-blowNash equivalence via an equivariant blow-Nash isomorphism, between the invariant Nash germs of the list of Theorem 2.1.

Equivariant blow-Nash equivalence (resp. equivariant blow-Nash equivalence via an equivariant blow-Nash isomorphism) is a particular case of the blow-Nash equivalence (resp. blowNash equivalence via a blow-Nash isomorphism) defined in [10]. In [13], Fichou proved that the classification of simple Nash germs $\left(\mathbb{R}^{n}, 0\right) \rightarrow(\mathbb{R}, 0)$ with respect to blow-Nash equivalence via a blow-Nash isomorphism is the same as Arnold's $A D E$-classification of real analytic germs with respect to right analytic equivalence.

As a consequence, the $A, D, E$-singularities, belonging to different blow-Nash classes, cannot be $G$-blow-Nash-equivalent via an equivariant blow-Nash isomorphism either. We are then reduced to try to distinguish the invariant germs of the families $A_{k}$ and $B_{k}$, resp. $C_{k}$ and $D_{k}$, $E_{6}$ and $F_{4}$.

For this purpose, we will use the equivariant zeta functions defined in [23], which are invariants for equivariant blow-Nash equivalence via an equivariant blow-Nash isomorphism. 


\section{Equivariant zeta functions}

Let $f:\left(\mathbb{R}^{n}, 0\right) \rightarrow(\mathbb{R}, 0)$ be an invariant Nash germ. We recall the definition given in [23] of the equivariant zeta functions of $f$.

Denote $\mathcal{L}:=\left\{\gamma:(\mathbb{R}, 0) \rightarrow\left(\mathbb{R}^{n}, 0\right) \mid \gamma(t)=a_{1} t+a_{2} t^{2}+\ldots, a_{i} \in \mathbb{R}^{n}\right\}$ the space of formal arcs at the origin of $\mathbb{R}^{n}$. The action of $G$ on $\mathbb{R}^{n}$ induces naturally an action of $G$ on $\mathcal{L}$, by left composition with $s$. For $m \in \mathbb{N} \backslash\{0\}$, the space

$$
\mathcal{L}_{m}:=\left\{\gamma:(\mathbb{R}, 0) \rightarrow\left(\mathbb{R}^{n}, 0\right) \mid \gamma(t)=a_{1} t+a_{2} t^{2}+\ldots+a_{m} t^{m}\right\}
$$

of arcs truncated at order $m+1$ is globally stable under this action, as well as the spaces

$$
\begin{gathered}
A_{m}(f):=\left\{\gamma \in \mathcal{L}_{m} \mid f \circ \gamma(t)=c t^{m}+\ldots, c \neq 0\right\} \\
A_{m}^{+}(f):=\left\{\gamma \in \mathcal{L}_{m} \mid f \circ \gamma(t)=+t^{m}+\ldots\right\} \text { and } A_{m}^{-}(f):=\left\{\gamma \in \mathcal{L}_{m} \mid f \circ \gamma(t)=-t^{m}+\ldots\right\} .
\end{gathered}
$$

These latter sets are Zariski constructible sets equipped with an algebraic action of $G$ and we define

$$
Z_{f}^{G}(u, T):=\sum_{m \geq 1} \beta^{G}\left(A_{m}(f)\right) u^{-m n} T^{m} \in \mathbb{Z}[u]\left[\left[u^{-1}\right]\right][[T]]
$$

and

$$
Z_{f}^{G, \pm}(u, T):=\sum_{m \geq 1} \beta^{G}\left(A_{m}^{ \pm}(f)\right) u^{-m n} T^{m} \in \mathbb{Z}[u]\left[\left[u^{-1}\right]\right][[T]]
$$

respectively the naive equivariant zeta function and the equivariant zeta functions with sign of $f$.

Here, $\beta^{G}(\cdot)$ denotes the equivariant virtual Poincaré series on $G-\mathcal{A S}$ sets of [14] : it is an additive invariant with respect to equivariant isomorphisms, with values in $\mathbb{Z}[[u]]$, such that, if $X$ is a compact nonsingular $G-\mathcal{A S}$ set, $\beta^{G}(X)=\sum_{i \in \mathbb{Z}} \operatorname{dim}_{\mathbb{Z}_{2}} H_{i}(X ; G) u^{i}$, where $H_{*}(X ; G)$ denotes the equivariant Borel-Moore homology of $X$ with coefficients in $\mathbb{Z}_{2}$ defined in [26].

Remark 4.1. - By an isomorphism between arc-symmetric sets is meant a birational map containing the arc-symmetric sets in its support.

- The equivariant virtual Poincaré series of a point is $\frac{u}{u-1}$, the equivariant virtual Poincaré series of two fixed points is $2 \frac{u}{u-1}$ and the equivariant virtual Poincaré series of two points exchanged by $G$ is 1 : see [14] Example 3.12.

- If $S^{d}$ denotes the unit sphere in $\mathbb{R}^{d}$ then

$$
\beta^{G}\left(S^{d}\right)=\left\{\begin{array}{l}
1+u+\ldots+u^{d} \text { if } G \text { acts via the central symmetry of } \mathbb{R}^{d} \\
2 \frac{u}{u-1}+u+\ldots+u^{d} \text { if } G \text { acts with a fixed point }
\end{array}\right.
$$

(see [14] Example 3.12).

- If $\mathbb{R}^{d}$ is equipped with any algebraic action of $G$, then $\beta^{G}\left(\mathbb{R}^{d}\right)=\frac{u^{d+1}}{u-1}$ : see [14] Example 3.12 . 
- If $X$ is a $G$ - $\mathcal{A S}$ set and if the affine space $\mathbb{R}^{d}$ is equipped with any algebraic action of $G$, then $\beta^{G}\left(X \times \mathbb{R}^{d}\right)=u^{d} \beta^{G}(X)$ (the product $X \times \mathbb{R}^{d}$ is equipped with the diagonal action of $G)$ : see [14] Proposition 3.13.

- If $X$ is a $G-\mathcal{A S}$ set and if the affine line $\mathbb{R}$ is equipped with an algebraic action of $G$ stabilizing 0 , then $\beta^{G}\left(X \times\left(\mathbb{R}^{*}\right)^{d}\right)=(u-1)^{d} \beta^{G}(X)$ : see [23] Lemma 3.9.

- If $X$ is a $G-\mathcal{A S}$, then the coefficients of the negative powers of $u$ in $\beta^{G}(X)$ are all equal to $\sum_{i \geq 0} \beta_{i}\left(X^{G}\right)$, where $X^{G}$ is the fixed point set of $X$ and $\beta_{i}(\cdot)$ denotes the $i^{t h}$ virtual Betti number ([21]) : see [14] Proposition 4.5.

Theorem 4.2 (Theorem 4.1 of [23]). Let $f, h:\left(\mathbb{R}^{n}, 0\right) \rightarrow(\mathbb{R}, 0)$ be two invariant Nash germs. If $f$ and $h$ are $G$-blow-Nash equivalent via an equivariant blow-Nash isomorphism, then $Z_{f}^{G}(u, T)=Z_{h}^{G}(u, T)$ and $Z_{f}^{G, \pm}(u, T)=Z_{h}^{G, \pm}(u, T)$.

Remark 4.3. In the rest of the paper, we will simply talk about equivariant blow-Nash equivalence to refer to equivariant blow-Nash equivalence via an equivariant blow-Nash isomorphism.

In the next parts of the paper, we are then going to use the equivariant zeta functions in order to try to distinguish the families $A_{k}$ and $B_{k}$, resp. $C_{k}$ and $D_{k}, E_{6}$ and $F_{4}$, with respect to $G$-blow-Nash equivalence. More precisely, we will show that, in some cases, some terms of the respective equivariant zeta functions of the considered germs are different.

On the other hand, we will prove that, in some other cases, the equivariant zeta functions are equal.

Before this, in the following section, we compute equivariant virtual Poincaré series associated to the quadratic form

$$
Q_{p, q}(y):=\sum_{i=1}^{p} y_{i}^{2}-\sum_{j=1}^{q} y_{p+j}^{2},
$$

where $p, q \in \mathbb{N},\left(y_{1}, \ldots, y_{p+q}\right) \in \mathbb{R}^{p+q}$. More precisely, we compute the equivariant virtual Poincaré series of the algebraic sets

$$
Y_{p, q}:=\left\{Q_{p, q}=0\right\} \text { and } Y_{p, q}^{\xi}:=\left\{Q_{p, q}=\xi\right\}
$$

for $\xi= \pm 1$, in the cases where the action of $G$ on $\mathbb{R}^{p+q}$ is given by

1. $\left(y_{1}, \ldots, y_{p}, y_{p+1}, \ldots, y_{p+q}\right) \mapsto\left(-y_{1}, \ldots, y_{p}, y_{p+1}, \ldots, y_{p+q}\right)$,

2. $\left(y_{1}, \ldots, y_{p}, y_{p+1}, \ldots, y_{p+q}\right) \mapsto\left(y_{1}, \ldots, y_{p},-y_{p+1}, \ldots, y_{p+q}\right)$,

3. $\left(y_{1}, \ldots, y_{p}, y_{p+1}, \ldots, y_{p+q}\right) \mapsto\left(-y_{1}, \ldots,-y_{p},-y_{p+1}, \ldots,-y_{p+q}\right)$,

4. or $\left(y_{1}, \ldots, y_{p}, y_{p+1}, \ldots, y_{p+q}\right) \mapsto\left(y_{1}, \ldots, y_{p}, y_{p+1}, \ldots, y_{p+q}\right)$.

This will reveal useful in the comparisons of the equivariant zeta functions. 


\section{Computation of $\beta^{G}\left(Y_{p, q}\right)$ and $\beta^{G}\left(Y_{p, q}^{\xi}\right)$}

Suppose that $p \leq q$. We have the following result :

Proposition 5.1. 1. If $0<p<q$, then

$$
\beta^{G}\left(Y_{p, q}\right)= \begin{cases}\frac{u^{p+q}-u^{q}+u^{p-1}}{u-1} & \text { in the case } n^{o} 1, \\ \frac{u^{p+q}-u^{q}+u^{p+1}}{u-1} & \text { in the three other cases. }\end{cases}
$$

2. If $p=q \neq 0$, then

$$
\beta^{G}\left(Y_{p, q}\right)= \begin{cases}\frac{u^{2 p}-u^{p}+u^{p-1}}{u-1} & \text { in the cases } n^{\circ} 1 \text { and } n^{\circ} \mathscr{Q}, \\ \frac{u^{2 p}-u^{p}+u^{p+1}}{u-1} & \text { in the two other cases. }\end{cases}
$$

3. If $p=0$, then

$$
\beta^{G}\left(Y_{p, q}\right)=\frac{u}{u-1} .
$$

Remark 5.2. If $q \leq p$, just exchange the roles of $p$ and $q$ along with the actions of the cases $\mathrm{n}^{\circ} 1$ and $\mathrm{n}^{\mathrm{O}} 2$.

Proof (of Proposition 5.1). If $p=0$, then $Y_{p, q}=\{0\}$ and $\beta^{G}\left(Y_{p, q}\right)=\frac{u}{u-1}$ by remark 4.1.

If $0<p<q$, as in [12] Proof of Proposition 2.1 and [13] Proof of Lemma 3.1, we apply the equivariant change of variables $u_{i}=y_{i}+y_{i+p}, v_{i}=y_{i}-y_{i+p}$ for $i=2, \ldots, p$ and the equation $Q_{p, q}=0$ becomes

$$
y_{1}^{2}-y_{p+1}^{2}+\sum_{i=2}^{p} u_{i} v_{i}-\sum_{j=2 p+1}^{p+q} y_{j}^{2}=0
$$

the action of $G$ on the new coordinates $u_{i}, v_{i}$ being trivial in the cases $\mathrm{n}^{\circ} 1, \mathrm{n}^{\circ} 2$ and $\mathrm{n}^{\circ} 4$, and changing their signs in the case $\mathrm{n}^{\circ} 3$.

As in [12] and [13], we write, by additivity of the equivariant virtual Poincaré series,

$$
\beta^{G}\left(Y_{p, q}\right)=\beta^{G}\left(Y_{p, q} \cap\left\{u_{2} \neq 0\right\}\right)+\beta^{G}\left(Y_{p, q} \cap\left\{u_{2}=0\right\}\right) .
$$

Because, if $u_{2} \neq 0$, the coordinate $v_{2}$ is determined via an equivariant isomorphism by $u_{2}$ and the other variables which are free, we have $\beta^{G}\left(Y_{p, q} \cap\left\{u_{2} \neq 0\right\}\right)=\beta^{G}\left(\mathbb{R}^{*} \times \mathbb{R}^{p+q-2}\right)=$ $(u-1) \frac{u^{p+q-1}}{u-1}$ (see remark 4.1). Furthermore, the equation describing $Y_{p, q} \cap\left\{u_{2}=0\right\}$ is

$$
y_{1}^{2}-y_{p+1}^{2}+\sum_{i=3}^{p} u_{i} v_{i}-\sum_{j=2 p+1}^{p+q} y_{j}^{2}=0
$$

(notice that the variable $v_{2}$ is then free) and, by an induction, we obtain

$$
\begin{aligned}
\beta^{G}\left(Y_{p, q}\right) & =\sum_{i=2}^{p} u^{p+q+1-i}+u^{p-1} \beta^{G}\left(\left\{y_{1}^{2}-y_{p+1}^{2}-\sum_{j=2 p+1}^{p+q} y_{j}^{2}=0\right\}\right) \\
& =u^{q+1} \frac{u^{p-1}-1}{u-1}+u^{p-1} \beta^{G}\left(\left\{y_{1}^{2}-y_{p+1}^{2}-\sum_{j=2 p+1}^{p+q} y_{j}^{2}=0\right\}\right) .
\end{aligned}
$$


Now, in order to compute $\beta^{G}\left(\left\{y_{1}^{2}-y_{p+1}^{2}-\sum_{j=2 p+1}^{p+q} y_{j}^{2}=0\right\}\right)$, we equivariantly blow up the latter algebraic set at the origin of $\mathbb{R}^{q-p+2}$ : in the chart $y_{1}=w, y_{i}=w z_{i}, i=p+1,2 p+$ $1, \ldots, p+q$, the blown-up variety is defined by

$$
w^{2}\left(1-z_{p+1}^{2}-\sum_{j=2 p+1}^{p+q} z_{j}^{2}\right)=0
$$

the action of $G$ being given by

- $\left(w, z_{p+1}, z_{2 p+1}, \ldots, z_{p+q}\right) \mapsto\left(-w,-z_{p+1},-z_{2 p+1}, \ldots,-z_{p+q}\right)$ in the case $\mathrm{n}^{\circ} 1$,

- $\left(w, z_{p+1}, z_{2 p+1}, \ldots, z_{p+q}\right) \mapsto\left(w,-z_{p+1}, z_{2 p+1}, \ldots, z_{p+q}\right)$ in the case $\mathrm{n}^{\circ} 2$,

- $\left(w, z_{p+1}, z_{2 p+1}, \ldots, z_{p+q}\right) \mapsto\left(-w, z_{p+1}, z_{2 p+1}, \ldots, z_{p+q}\right)$ in the case $\mathrm{n}^{\mathrm{o}} 3$,

- $\left(w, z_{p+1}, z_{2 p+1}, \ldots, z_{p+q}\right) \mapsto\left(w, z_{p+1}, z_{2 p+1}, \ldots, z_{p+q}\right)$ in the case $\mathrm{n}^{\mathrm{o}} 4$.

We have

$$
\begin{aligned}
\beta^{G}\left(\left\{y_{1}^{2}-y_{p+1}^{2}-\sum_{j=2 p+1}^{p+q} y_{j}^{2}=0\right\} \backslash\{0\}\right) & =\beta^{G}\left(\left\{1-z_{p+1}^{2}-\sum_{j=2 p+1}^{p+q} z_{j}^{2}=0\right\} \backslash\{w=0\}\right) \\
& =\beta^{G}\left(\mathbb{R}^{*} \times S^{q-p}\right) \\
& =(u-1) \beta^{G}\left(S^{q-p}\right)
\end{aligned}
$$

Finally, since the action of $G$ on the sphere $S^{q-p}$ is the central symmetry in the case $\mathrm{n}^{\circ} 1$ and admits a fixed point in the three other cases, we have

$$
\beta^{G}\left(S^{q-p}\right)=\left\{\begin{array}{l}
\frac{u^{q-p+1}-1}{u-1} \text { in the case } \mathrm{n}^{\circ} 1, \\
\frac{u^{q-p+1}+u}{u-1} \text { in the three other cases }
\end{array}\right.
$$

(see remark 4.1). Using the additivity relation

$$
\beta^{G}\left(\left\{y_{1}^{2}-y_{p+1}^{2}-\sum_{j=2 p+1}^{p+q} y_{j}^{2}=0\right\} \backslash\{0\}\right)=\beta^{G}\left(\left\{y_{1}^{2}-y_{p+1}^{2}-\sum_{j=2 p+1}^{p+q} y_{j}^{2}=0\right\}\right)-\beta^{G}(\{0\})
$$

and the equality $\beta^{G}(\{0\})=\frac{u}{u-1}$, we obtain the desired result.

If $p=q \in \mathbb{N} \backslash\{0 ; 1\}$, we do as before in order to obtain the equality

$$
\beta^{G}\left(Y_{p, q}\right)=u^{p+1} \frac{u^{p-1}-1}{u-1}+u^{p-1} \beta^{G}\left(\left\{y_{1}^{2}-y_{p+1}^{2}=0\right\}\right)
$$

(notice that the quantity $\beta^{G}\left(\left\{y_{1}^{2}-y_{p+1}^{2}=0\right\}\right)$ is the same in the cases $\mathrm{n}^{\circ} 1$ and $\mathrm{n}^{\circ} 2$ ). Now, as above, we equivariantly blow up at the origin of $\mathbb{R}^{2}$ and look in the chart $y_{1}=u_{1}, y_{p+1}=$ $u_{1} v_{p+1}$ : the blown-up variety is given by the equation

$$
u_{1}^{2}\left(1-v_{p+1}^{2}\right)=0
$$

and the action of $G$ is given by 
- $\left(u_{1}, v_{p+1}\right) \mapsto\left(-u_{1},-v_{p+1}\right)$ in the case $\mathrm{n}^{\circ} 1$,

- $\left(u_{1}, v_{p+1}\right) \mapsto\left(u_{1},-v_{p+1}\right)$ in the case $\mathrm{n}^{\mathrm{o}} 2$,

- $\left(u_{1}, v_{p+1}\right) \mapsto\left(-u_{1}, v_{p+1}\right)$ in the case $\mathrm{n}^{\mathrm{o}} 3$,

- $\left(u_{1}, v_{p+1}\right) \mapsto\left(u_{1}, v_{p+1}\right)$ in the case $\mathrm{n}^{\circ} 4$.

As a consequence,

$$
\beta^{G}\left(\left\{1-v_{p+1}^{2}=0\right\}\right)=\left\{\begin{array}{l}
1 \text { in the cases } \mathrm{n}^{\circ} 1 \text { and } \mathrm{n}^{\mathrm{o}} 2, \\
2 \frac{u}{u-1} \text { in the two other cases }
\end{array}\right.
$$

(see remark 4.1) and we obtain the desired result.

If $p=q=1$, we have $\beta^{G}\left(Y_{p, q}\right)=\beta^{G}\left(\left\{y_{1}^{2}-y_{p+1}^{2}=0\right\}\right)$ and we can use the previous computation.

This proposition can be used to compute the quantities $\beta^{G}\left(Y_{p, q}^{\xi}\right)$. Indeed :

Proposition 5.3. We have

$$
\beta^{G}\left(Y_{p, q}^{+1}\right)=\frac{1}{u-1}\left(\beta^{G}\left(Y_{p, q+1}\right)-\beta^{G}\left(Y_{p, q}\right)\right)
$$

and

$$
\beta^{G}\left(Y_{p, q}^{-1}\right)=\frac{1}{u-1}\left(\beta^{G}\left(Y_{p+1, q}\right)-\beta^{G}\left(Y_{p, q}\right)\right)
$$

Remark 5.4. We have the same equalities if $q \leq p$.

Proof (of Proposition 5.3). We show the first equality, the proof of the second one being similar.

Denote $Z_{p, q}$ the projective algebraic set

$$
\left\{\left[Y_{1}: \ldots: Y_{p+q}\right] \in \mathbb{P}^{p+q-1}(\mathbb{R}) \mid \sum_{i=1}^{p} Y_{i}^{2}-\sum_{j=1}^{q} Y_{p+j}^{2}=0\right\}
$$

As in [11] Proof of Corollary 2.5, we can equivariantly compactify $Y_{p, q}^{+1}$ into the projective algebraic set $Z_{p, q+1}$, the part at infinity being equivariantly isomorphic to $Z_{p, q}$ (we equip $\mathbb{P}^{p+q}(\mathbb{R})$ and $\mathbb{P}^{p+q-1}(\mathbb{R})$ with the actions of $G$ naturally induced from the considered action on the variables of $\left.\mathbb{R}^{p+q}\right)$.

Now, we compute $\beta^{G}\left(Z_{p, q}\right)$, using, as in [11] Proof of Proposition 2.1, the fact that the projection

$$
p: \begin{array}{ccc}
Y_{p, q} \backslash\{0\} & \rightarrow & Z_{p, q} \\
\left(y_{1}, \ldots, y_{p+q}\right) & \mapsto & {\left[y_{1}: \ldots: y_{p+q}\right]}
\end{array}
$$


is a piecewise algebraically trivial fibration, compatible with the respective considered actions of $G$. More precisely, we can cover $Z_{p, q}$ by the globally $G$-invariant open subvarieties

$$
U_{i}:=Z_{p, q} \cap\left\{Y_{i} \neq 0\right\}, i \in\{1, \ldots, p+q\}
$$

and, for each $i \in\{1, \ldots, p+q\}$, we can define the isomorphism

$$
\begin{array}{rlc}
\varphi_{i}: p^{-1}\left(U_{i}\right)=Y_{p, q} \cap\left\{y_{i} \neq 0\right\} & \rightarrow & U_{i} \times \mathbb{R}^{*} \\
\left(y_{1}, \ldots, y_{i}, \ldots, y_{p+q}\right) & \mapsto & \left(\left[y_{1}: \ldots: y_{i}: \ldots: y_{p+q}\right], y_{i}\right)
\end{array}
$$

For $i \in\{1, \ldots, p+q\}$, if the sign of the coordinate $y_{i}$ is changed under the action of $G$, we equip $\mathbb{R}^{*}$ with the action of $G$ given by the involution $z \mapsto-z$. If $y_{i}$ remains unchanged under the action of $G$, we equip $\mathbb{R}^{*}$ with the trivial action of $G$. Furthermore equipping the product $U_{i} \times \mathbb{R}^{*}$ with the diagonal action, this makes the isomorphism $\varphi_{i}$ equivariant.

By the additivity of the equivariant virtual Poincaré series, the quantity $\beta^{G}\left(Y_{p, q} \backslash\{0\}\right)$ can be written as the alternated sum of the terms

$$
\sum_{J \subset\{1, \ldots, p+q\}, \operatorname{Card}(J)=r} \beta^{G}\left(p^{-1}\left(\bigcap_{m \in J} U_{m}\right)\right), 1 \leq r \leq p+q,
$$

and, via the equivariant isomorphisms $\varphi_{i}$, we have

$$
\beta^{G}\left(p^{-1}\left(\bigcap_{m \in J} U_{m}\right)\right)=\beta^{G}\left(\left(\bigcap_{m \in J} U_{m}\right) \times \mathbb{R}^{*}\right)=(u-1) \beta^{G}\left(\bigcap_{m \in J} U_{m}\right) .
$$

As a consequence, once again thanks to the additivity of the equivariant virtual Poincaré series,

$$
\beta^{G}\left(Y_{p, q} \backslash\{0\}\right)=(u-1) \beta^{G}\left(Z_{p, q}\right) .
$$

Therefore,

$$
\begin{aligned}
\beta^{G}\left(Y_{p, q}^{+1}\right) & =\beta^{G}\left(Z_{p, q+1}\right)-\beta^{G}\left(Z_{p, q}\right) \\
& =\frac{1}{u-1}\left(\beta^{G}\left(Y_{p, q+1} \backslash\{0\}\right)-\beta^{G}\left(Y_{p, q} \backslash\{0\}\right)\right) \\
& =\frac{1}{u-1}\left(\beta^{G}\left(Y_{p, q+1}\right)-\beta^{G}\left(Y_{p, q}\right)\right) .
\end{aligned}
$$

\section{The germs $A_{k}$ and $B_{k}$}

In this section, we want to study the relations with respect to $G$-blow-Nash equivalence between the invariant germs of the families

$$
f_{k}^{\epsilon_{k}}(x):= \pm x_{1}^{2}+\epsilon_{k} x_{2}^{k+1}+Q \quad \text { and } g_{k}^{\epsilon_{k}}(x):=\epsilon_{k} x_{1}^{2 k} \pm x_{2}^{2}+Q^{\prime},
$$


where $\epsilon_{k} \in\{-1 ;+1\}$.

First, if any two invariant Nash germs are $G$-blow-Nash equivalent, they are in particular blow-Nash equivalent and then, according to [11] Theorem 2.5, they have the same corank and index.

Therefore, if two germs $f_{k}^{\epsilon_{k}}$ and $f_{l}^{\epsilon_{l}}$ are $G$-blow-Nash equivalent, they have the same quadratic part up to permutation of the variables $x_{1}, x_{3}, \ldots, x_{n}$. Furthermore, we know, by [13] Proposition 3.4, that $k=l$ and, if $k=l$ is odd, that $\epsilon_{k}=\epsilon_{l}$. If $k$ is even, $f_{k}^{+1}\left(x_{1}, x_{2}, x_{3}, \ldots, x_{n}\right)=f_{k}^{-1}\left(x_{1},-x_{2}, x_{3}, \ldots, x_{n}\right)$ and the (linear) change of variables is equivariant with respect to the involution $s$ on $\mathbb{R}^{n}: f_{k}^{+1}$ and $f_{k}^{-1}$ are then $G$-Nash equivalent, in particular $G$-blow-Nash equivalent.

As a conclusion, inside the family $A_{k}$, we are reduced to try to distinguish the germs

$$
f_{k}^{\epsilon_{k},+}(x):=+x_{1}^{2}+\epsilon_{k} x_{2}^{k+1}+Q \text { and } f_{k}^{\epsilon_{k},-}(x):=-x_{1}^{2}+\epsilon_{k} x_{2}^{k+1}+Q^{\prime}
$$

where $\epsilon_{k} \in\{-1 ;+1\}$ and $+x_{1}^{2}+Q$ and $-x_{1}^{2}+Q^{\prime}$ are the same quadratic part up to permutation of the variables $x_{1}, x_{3}, \ldots, x_{n}$.

Similarly, if two germs $g_{k}^{\epsilon_{k}}$ and $g_{l}^{\epsilon_{l}}$ are $G$-blow-Nash equivalent, they have the same quadratic part, up to permutation of the variables $x_{2}, \ldots, x_{n}$, and $k=l$ and $\epsilon_{k}=\epsilon_{l}$.

Finally, if two germs $f_{k}^{\epsilon_{k}}$ and $g_{k^{\prime}}^{\epsilon_{k^{\prime}}}$ are blow-Nash equivalent, then $k=2 k^{\prime}-1$ and $\epsilon_{k}=\epsilon_{k^{\prime}}$, and furthermore $\pm x_{1}^{2}+Q$ and $\pm x_{2}^{2}+Q^{\prime}$ are the same quadratic part up to permutation of all variables. Consequently, it remains to look at the relation between the germs

$$
f_{2 k-1}=\epsilon x_{2}^{2 k}+\eta x_{1}^{2}+Q \text { and } g_{k}=\epsilon x_{1}^{2 k}+\eta^{\prime} x_{2}^{2}+Q^{\prime}
$$

where $\epsilon, \eta, \eta^{\prime} \in\{1,-1\}$ and $\eta x_{1}^{2}+Q=\eta^{\prime} x_{2}^{2}+Q^{\prime}$ up to permutation of all variables.

In the following parts of this section, we will compute some terms of the equivariant zeta functions of $f_{k}$ and $g_{k}$. In virtue of theorem 4.2, this will allow us to make further distinctions inside each of the above couples of germs in some cases.

\subsection{Computation of the first terms of the equivariant zeta functions}

If $h$ is an invariant Nash germ $\left(\mathbb{R}^{n}, 0\right) \rightarrow(\mathbb{R}, 0)$, recall that, for $m \geq 1$,

$$
\begin{aligned}
A_{m}(h) & =\left\{\gamma(t)=a_{1} t+\cdots+a_{m} t^{m} \in \mathcal{L}_{m} \mid h \circ \gamma(t)=c t^{m}+\cdots, c \neq 0\right\} \\
& =\left\{\gamma \in \mathcal{L}_{m} \mid h \circ \gamma(t)=c t^{m}+\cdots, c \in \mathbb{R}\right\} \backslash\left\{\gamma \in \mathcal{L}_{m} \mid h \circ \gamma(t)=0 \times t^{m}+\cdots\right\}
\end{aligned}
$$

Since $h$ is an invariant germ, the latter sets are both globally stable under the action of $G$ on $\mathcal{L}_{m}$ and, by the additivity of the equivariant virtual Poincare series, the quantity $\beta^{G}\left(A_{m}(h)\right)$ is equal to the difference $\beta^{G}\left({ }^{0} A_{m}(h)\right)-\beta^{G}\left(A_{m}^{0}(h)\right)$, where

${ }^{0} A_{m}(h):=\left\{\gamma \in \mathcal{L}_{m} \mid h \circ \gamma(t)=c t^{m}+\cdots, c \in \mathbb{R}\right\}$ and $A_{m}^{0}(h):=\left\{\gamma \in \mathcal{L}_{m} \mid h \circ \gamma(t)=0 \times t^{m}+\cdots\right\}$. 
Fix $k \geq 0$ and consider the invariant germ $f_{k}^{\epsilon, \eta}\left(x_{1}, \ldots, x_{n}\right)=\eta x_{1}^{2}+\epsilon x_{2}^{k+1}+Q$. We denote $x_{2}=x$ and $\eta x_{1}^{2}+Q=Q_{p, q}=\sum_{i=1}^{p} y_{i}^{2}-\sum_{j=1}^{q} y_{p+j}^{2}$ in such a way that $G$ acts on the renamed coordinates via the involution $\mathrm{n}^{\mathrm{o}} 1$ or $\mathrm{n}^{\mathrm{o}} 2$ depending on the sign of $\eta$.

We first compute $\beta^{G}\left(A_{m}^{0}\left(f_{k}^{\epsilon, \eta}\right)\right)$ for $m<k+1$. Notice that the set $A_{1}\left(f_{1}^{\epsilon, \eta}\right)$ is empty and, consequently, $\beta^{G}\left(A_{1}\left(f_{1}^{\epsilon, \eta}\right)\right)=0$.

Proposition 6.1. Suppose $k \geq 2$ and $m<k+1$.

1. If $p q=0$, then

$$
\beta^{G}\left(A_{m}^{0}\left(f_{k}^{\epsilon, \eta}\right)\right)=\left\{\begin{array}{l}
\frac{u^{m+(r+1)(p+q)+1}}{u-1} \text { if } m=2 r+1, \\
\frac{u^{m+r(p+q)+1}}{u-1} \text { if } m=2 r .
\end{array}\right.
$$

2. If $(p, q)=(1,1)$, then

$$
\beta^{G}\left(A_{m}^{0}\left(f_{k}^{\epsilon, \eta}\right)\right)=\left\{\begin{array}{l}
r u^{2 m} \beta^{G}\left(Y_{1,1} \backslash\{0\}\right)+\frac{u^{4(r+1)}}{u-1} \text { if } m=2 r+1, \\
(r-1) u^{2 m} \beta^{G}\left(Y_{1,1} \backslash\{0\}\right)+u^{4 r} \beta^{G}\left(Y_{1,1}\right) \text { if } m=2 r,
\end{array}\right.
$$

3. If $p q \neq 0$ and $(p, q) \neq(1,1)$, then

$$
\beta^{G}\left(A_{m}^{0}\left(f_{k}^{\epsilon, \eta}\right)\right)=\left\{\begin{array}{l}
u^{m} u^{(r+1)(p+q)-1} \frac{u^{r(p+q-2)}-1}{u^{p+q-2}-1} \beta^{G}\left(Y_{p, q} \backslash\{0\}\right)+\frac{u^{(r+1)(2+p+q)}}{u-1} \text { if } m=2 r+1, \\
u^{m} u^{(r+1)(p+q)-2} \frac{u^{(r-1)(p+q-2)}-1}{u^{p+q-2}-1} \beta^{G}\left(Y_{p, q} \backslash\{0\}\right)+u^{r(2+p+q)} \beta^{G}\left(Y_{p, q}\right) \text { if } m=2 r .
\end{array}\right.
$$

Proof. We follow the computation steps of [13], keeping the track of the action of $G$ in our context.

An $\operatorname{arc} \gamma$ of $\mathcal{L}_{m}$ can be written as

$$
\begin{aligned}
\gamma(t) & =\left(a_{1} t+\cdots+a_{m} t^{m}, c_{1}^{1} t+\cdots+c_{m}^{1} t^{m}, \ldots, c_{1}^{p+q} t+\cdots+c_{m}^{p+q} t^{m}\right) \\
& =\left(\begin{array}{c}
a_{1} \\
c_{1}^{1} \\
\vdots \\
c_{1}^{p+q}
\end{array}\right) t+\cdots+\left(\begin{array}{c}
a_{m} \\
c_{m}^{1} \\
\vdots \\
c_{m}^{p+q}
\end{array}\right) t^{m}=\left(\begin{array}{c}
a_{1} \\
c_{1}
\end{array}\right) t+\cdots+\left(\begin{array}{c}
a_{m} \\
c_{m}
\end{array}\right) t^{m}
\end{aligned}
$$

if $c_{i}:=\left(c_{i}^{1}, \ldots, c_{i}^{p+q}\right)$. The group $G$ acts on $\mathcal{L}_{m}$ changing the sign of the variables $c_{i}^{1}$, resp. $c_{i}^{p+1}$, in the case $\mathrm{n}^{\mathrm{o}} 1$, resp. $\mathrm{n}^{\mathrm{o}} 2$.

We begin with the case $p q \neq 0,(p, q) \neq(1,1)$ and $m=2 r+1$ odd. An arc $\gamma$ of $\mathcal{L}_{m}$ belongs to $A_{m}^{0}\left(f_{k}^{\epsilon, \eta}\right)$ if and only if

$$
\left\{\begin{array}{l}
Q_{p, q}\left(c_{1}\right)=0 \\
\Phi_{p, q}\left(c_{1}, c_{2}\right)=0 \\
\cdots \\
Q_{p, q}\left(c_{r}\right)+\sum_{t=1}^{r-1} \Phi_{p, q}\left(c_{t}, c_{2 r-t}\right)=0 \\
\sum_{t=1}^{r} \Phi_{p, q}\left(c_{t}, c_{2 r+1-t}\right)=0
\end{array}\right.
$$


where $\Phi_{p, q}$ is the function on $\mathbb{R}^{p+q} \times \mathbb{R}^{p+q}$ defined by $\Phi_{p, q}(u, v)=2 \sum_{i=1}^{p} u_{i} v_{i}-2 \sum_{j=1}^{q} u_{p+j} v_{p+j}$.

The first equality of the system means $c_{1} \in Y_{p, q}$ by definition. Now, if $c_{1}^{1} \neq 0$, the variables $c_{2}^{1}, \ldots, c_{2 r}^{1}$ are determined by $c_{1}^{1}$ and the other (free) variables via an equivariant morphism. Therefore,

$$
\begin{aligned}
\beta^{G}\left(A_{m}^{0}\left(f_{k}^{\epsilon, \eta}\right)\right) & =\beta^{G}\left(A_{m}^{0}\left(f_{k}^{\epsilon, \eta}\right) \cap\left\{c_{1}^{1} \neq 0\right\}\right)+\beta^{G}\left(A_{m}^{0}\left(f_{k}^{\epsilon, \eta}\right) \cap\left\{c_{1}^{1}=0\right\}\right) \\
& =\beta^{G}\left(\left(Y_{p, q} \backslash\left(\{0\} \times Y_{p-1, q}\right)\right) \times \mathbb{R}^{m+(m-1)(p+q-1)+1}\right)+\beta^{G}\left(A_{m}^{0}\left(f_{k}^{\epsilon, \eta}\right) \cap\left\{c_{1}^{1}=0\right\}\right) \\
& =u^{m+(m-1)(p+q-1)+1} \beta^{G}\left(Y_{p, q} \backslash\left(\{0\} \times Y_{p-1, q}\right)\right)+\beta^{G}\left(A_{m}^{0}\left(f_{k}^{\epsilon, \eta}\right) \cap\left\{c_{1}^{1}=0\right\}\right)
\end{aligned}
$$

Next, we have

$\beta^{G}\left(A_{m}^{0}\left(f_{k}^{\epsilon, \eta}\right) \cap\left\{c_{1}^{1}=0\right\}\right)=u^{m+(m-1)(p+q-1)+1} \beta^{G}\left(Y_{p-1, q} \backslash\left(\{0\} \times Y_{p-2, q}\right)\right)+\beta^{G}\left(A_{m}^{0}\left(f_{k}^{\epsilon, \eta}\right) \cap\left\{c_{1}^{1}=c_{1}^{2}=0\right\}\right)$

and we obtain by induction

$$
\beta^{G}\left(A_{m}^{0}\left(f_{k}^{\epsilon, \eta}\right)\right)=u^{m+(m-1)(p+q-1)+1} \beta^{G}\left(Y_{p, q} \backslash\{0\}\right)+\beta^{G}\left(A_{m}^{0}\left(f_{k}^{\epsilon, \eta}\right) \cap\left\{c_{1}^{1}=\ldots=c_{1}^{p}=0\right\}\right) .
$$

If $c_{1}^{1}=\ldots=c_{1}^{p}=0$ then $c_{1}^{p+1}=\ldots=c_{1}^{p+q}=0$ (since $Q_{p, q}\left(c_{1}\right)=0$ ) and the other variables verify the system

$$
\left\{\begin{array}{l}
Q_{p, q}\left(c_{2}\right)=0, \\
\Phi_{p, q}\left(c_{2}, c_{3}\right)=0 \\
\cdots \\
Q_{p, q}\left(c_{r}\right)+\sum_{t=2}^{r-1} \Phi_{p, q}\left(c_{t}, c_{2 r-t}\right)=0 \\
\sum_{t=2}^{r} \Phi_{p, q}\left(c_{t}, c_{2 r+1-t}\right)=0 .
\end{array}\right.
$$

Noticing that the vector $c_{m}$ as well as the variables $a_{m-1}, a_{m}$ are free and renaming the remaining variables, we have

$$
\beta^{G}\left(A_{m}^{0}\left(f_{k}^{\epsilon, \eta}\right)\right)=u^{m+(m-1)(p+q-1)+1} \beta^{G}\left(Y_{p, q} \backslash\{0\}\right)+u^{2+(p+q)} \beta^{G}\left(A_{m-2}^{0}\left(f_{k}^{\epsilon, \eta}\right)\right)
$$

and, by an induction,

$\beta^{G}\left(A_{m}^{0}\left(f_{k}^{\epsilon, \eta}\right)\right)=\beta^{G}\left(Y_{p, q} \backslash\{0\}\right) \sum_{t=0}^{r-1} u^{t(2+p+q)} u^{m-2 t+(m-2 t-1)(p+q-1)+1}+u^{(r-1)(2+p+q)} \beta^{G}\left(A_{3}^{0}\left(f_{k}^{\epsilon, \eta}\right) \cap\left\{c_{1}=0\right\}\right)$

As a conclusion, since the system describing $A_{3}^{0}\left(f_{k}^{\epsilon, \eta}\right) \cap\left\{c_{1}=0\right\}$ is trivial, the variables $a_{i}$ as well as the vectors $c_{2}$ and $c_{3}$ are free and

$$
\beta^{G}\left(A_{m}^{0}\left(f_{k}^{\epsilon, \eta}\right)\right)=u^{m} u^{(r+1)(p+q)-1} \frac{u^{r(p+q-2)}-1}{u^{p+q-2}-1} \beta^{G}\left(Y_{p, q} \backslash\{0\}\right)+\frac{u^{(r+1)(2+p+q)}}{u-1} .
$$


If $m$ is even, $m=2 r$, the system describing $A_{m}^{0}\left(f_{k}^{\epsilon, \eta}\right)$ is

$$
\left\{\begin{array}{l}
Q_{p, q}\left(c_{1}\right)=0 \\
\Phi_{p, q}\left(c_{1}, c_{2}\right)=0 \\
\cdots \\
\sum_{t=1}^{r-1} \Phi_{p, q}\left(c_{t}, c_{2 r-1-t}\right)=0 \\
Q_{p, q}\left(c_{r}\right)+\sum_{t=1}^{r-1} \Phi_{p, q}\left(c_{t}, c_{2 r-t}\right)=0
\end{array}\right.
$$

Therefore, by similar computations, we obtain

$$
\beta^{G}\left(A_{m}^{0}\left(f_{k}^{\epsilon, \eta}\right)\right)=\beta^{G}\left(Y_{p, q} \backslash\{0\}\right) \sum_{t=0}^{r-2} u^{t(2+p+q)} u^{m-2 t+(m-2 t-1)(p+q-1)+1}+u^{(r-1)(2+p+q)} \beta^{G}\left(A_{2}^{0}\left(f_{k}^{\epsilon, \eta}\right)\right)
$$

Since $A_{2}^{0}\left(f_{k}^{\epsilon, \eta}\right)$ is described by the equation $Q_{p, q}\left(c_{1}\right)=0$, the vector $c_{2}$ as well as the variables $a_{1}$ and $a_{2}$ being free,

$$
\beta^{G}\left(A_{m}^{0}\left(f_{k}^{\epsilon, \eta}\right)\right)=u^{m} u^{(r+1)(p+q)-2} \frac{u^{(r-1)(p+q-2)}-1}{u^{p+q-2}-1} \beta^{G}\left(Y_{p, q} \backslash\{0\}\right)+u^{r(2+p+q)} \beta^{G}\left(Y_{p, q}\right) .
$$

Finally, if $(p, q)=(1,1)$, the same process gives

$$
\beta^{G}\left(A_{m}^{0}\left(f_{k}^{\epsilon, \eta}\right)\right)=\left\{\begin{array}{l}
\beta^{G}\left(Y_{1,1} \backslash\{0\}\right) \sum_{t=0}^{r-1} u^{2 m}+\frac{u^{4(r+1)}}{u-1} \text { if } m=2 r+1, \\
\beta^{G}\left(Y_{1,1} \backslash\{0\}\right) \sum_{t=0}^{r-2} u^{2 m}+u^{4 r} \beta^{G}\left(Y_{1,1}\right) \text { if } m=2 r .
\end{array}\right.
$$

If $p q=0$, since $Y_{p, q}=\{0\}$, the equations $Q_{p, q}\left(c_{1}\right)=\ldots=Q_{p, q}\left(c_{r}\right)=0$ impose $c_{1}, \ldots, c_{r}$ to be zero vectors and, the other variables being free, we have

$$
\beta^{G}\left(A_{m}^{0}\left(f_{k}^{\epsilon, \eta}\right)\right)=\left\{\begin{array}{l}
\frac{u^{m+(r+1)(p+q)+1}}{u-1} \text { if } m=2 r+1, \\
\frac{u^{m+r(p+q)+1}}{u-1} \text { if } m=2 r
\end{array}\right.
$$

Remark 6.2. We obtain the same quantities for $\beta^{G}\left(A_{m}^{0}\left(g_{l}^{\epsilon}\right)\right)$ with $m<2 l$, providing we equip the set $Y_{p, q}$ with the trivial action of $G$. Indeed, the computation steps above remain equivariant if the group $G$ acts on $\mathcal{L}_{m}$ changing the sign of the variables $a_{i}$.

Proposition 6.3. Let $h$ be an invariant Nash germ $\left(\mathbb{R}^{n}, 0\right) \rightarrow(\mathbb{R}, 0)$ and $m \geq 2$. Then

$$
\beta^{G}\left({ }^{0} A_{m}(h)\right)=u^{n} \beta^{G}\left(A_{m-1}^{0}(h)\right) .
$$

Proof. Notice that ${ }^{0} A_{m}(h)=\left\{\gamma \in \mathcal{L}_{m} \mid h \circ \gamma(t)=0 \times t+\cdots+0 \times t^{m-1}+c t^{m}+\cdots\right\}$. Therefore, the system describing ${ }^{0} A_{m}(h)$ is the same as the system describing $A_{m-1}^{0}(h)$, the last $n$ variables being free. 
As a consequence, we have an equivariant isomorphism between ${ }^{0} A_{m}(h)$ and the product $\mathbb{R}^{n} \times A_{m-1}^{0}(h)$ (this set is equipped with the diagonal action of $G$, the first term being equipped with the involution $s$ ) and consequently

$$
\beta^{G}\left({ }^{0} A_{m}(h)\right)=u^{n} \beta^{G}\left(A_{m-1}^{0}(h)\right) .
$$

We also compute $\beta^{G}\left(A_{m}^{\xi}\left(f_{k}^{\epsilon, \eta}\right)\right)$ for $m<k+1$ :

Proposition 6.4. Suppose $k \geq 2$ and $m<k+1$.

1. If $p q=0$, then

$$
\beta^{G}\left(A_{m}^{\xi}\left(f_{k}^{\epsilon, \eta}\right)\right)=\left\{\begin{array}{l}
0 \text { if } m=2 r+1, \\
u^{m+r(p+q)} \beta^{G}\left(Y_{p, q}^{\xi}\right) \text { if } m=2 r .
\end{array}\right.
$$

2. If $(p, q)=(1,1)$, then

$$
\beta^{G}\left(A_{m}^{\xi}\left(f_{k}^{\epsilon, \eta}\right)\right)=\left\{\begin{array}{l}
r u^{2 m} \beta^{G}\left(Y_{1,1} \backslash\{0\}\right) \text { if } m=2 r+1, \\
(r-1) u^{2 m} \beta^{G}\left(Y_{1,1} \backslash\{0\}\right)+u^{4 r} \beta^{G}\left(Y_{1,1}^{\xi}\right) \text { if } m=2 r,
\end{array}\right.
$$

3. If $p q \neq 0$ and $(p, q) \neq(1,1)$, then

$$
\beta^{G}\left(A_{m}^{\xi}\left(f_{k}^{\epsilon, \eta}\right)\right)=\left\{\begin{array}{l}
u^{m} u^{(r+1)(p+q)-1} \frac{u^{r(p+q-2)}-1}{u^{p+q-2}-1} \beta^{G}\left(Y_{p, q} \backslash\{0\}\right) \text { if } m=2 r+1, \\
u^{m} u^{(r+1)(p+q)-2} \frac{u^{(r-1)(p+q-2)}-1}{u^{p+q-2}-1} \beta^{G}\left(Y_{p, q} \backslash\{0\}\right)+u^{r(2+p+q)} \beta^{G}\left(Y_{p, q}^{\xi}\right) \text { if } m=2 r .
\end{array}\right.
$$

Proof. We first deal with the case $p q \neq 0,(p, q) \neq(1,1)$ and $m=2 r$ even. Keeping the notations of the proof of 6.1 , the system describing $A_{m}^{\xi}\left(f_{k}^{\epsilon, \eta}\right)$ is

$$
\left\{\begin{array}{l}
Q_{p, q}\left(c_{1}\right)=0, \\
\Phi_{p, q}\left(c_{1}, c_{2}\right)=0 \\
\cdots \\
\sum_{t=1}^{r-1} \Phi_{p, q}\left(c_{t}, c_{2 r-1-t}\right)=0 \\
Q_{p, q}\left(c_{r}\right)+\sum_{t=1}^{r-1} \Phi_{p, q}\left(c_{t}, c_{2 r-t}\right)=\xi
\end{array}\right.
$$

The computation steps are the same as in the proof of proposition 6.1 , and we have

$$
\beta^{G}\left(A_{m}^{\xi}\left(f_{k}^{\epsilon, \eta}\right)\right)=u^{m} u^{(r+1)(p+q)-2} \frac{u^{(r-1)(p+q-2)}-1}{u^{p+q-2}-1} \beta^{G}\left(Y_{p, q} \backslash\{0\}\right)+u^{(r-1)(2+p+q)} \beta^{G}\left(A_{2}^{\xi}\left(f_{k}^{\epsilon, \eta}\right)\right) .
$$

Since the set $A_{2}^{\xi}\left(f_{k}^{\epsilon, \eta}\right)$ is described by the equation $Q_{p, q}\left(c_{1}\right)=\xi$ and the other variables being free, we obtain the result.

If $m$ is odd, $m=2 r+1$, as in the proof of proposition 6.1 , we obtain

$$
\beta^{G}\left(A_{m}^{\xi}\left(f_{k}^{\epsilon, \eta}\right)\right)=u^{m} u^{(r+1)(p+q)-1} \frac{u^{r(p+q-2)}-1}{u^{p+q-2}-1} \beta^{G}\left(Y_{p, q} \backslash\{0\}\right)+u^{(r-1)(2+p+q)} \beta^{G}\left(A_{3}^{\xi}\left(f_{k}^{\epsilon, \eta}\right) \cap\left\{c_{1}=0\right\}\right)
$$

and the set $A_{3}^{\xi}\left(f_{k}^{\epsilon, \eta}\right) \cap\left\{c_{1}=0\right\}$ is empty.

Similar considerations provide the results for the cases $(p, q)=(1,1)$ and $p q=0$. 
Remark 6.5. Again, we have the same quantities for $\beta^{G}\left(A_{m}^{\xi}\left(g_{l}^{\epsilon}\right)\right)$ with $m<2 l$, providing we equip the sets $Y_{p, q}$ and $Y_{p, q}^{\xi}$ with the trivial action of $G$.

Now, we are ready to deduce distinctions, with respect to $G$-blow-Nash equivalence, between $f_{k}^{\epsilon,+}$ and $f_{k}^{\epsilon,-}$, respectively between $f_{2 k-1}$ and $g_{k}$, in some cases :

Corollary 6.6. Let $k \geq 1$. Suppose that the invariant germs

$$
f_{k}^{\epsilon,+}(x):=+x_{1}^{2}+\epsilon x_{2}^{k+1}+Q \text { and } f_{k}^{\epsilon,-}(x):=-x_{1}^{2}+\epsilon x_{2}^{k+1}+Q^{\prime}
$$

have the same quadratic part up to permutation of the variables $x_{1}, x_{3}, \ldots, x_{n}$. Then they are not G-blow-Nash equivalent.

Proof. We begin by assuming $k \geq 2$. We first compare $\beta^{G}\left(A_{2}\left(f_{k}^{\epsilon,+}\right)\right)$ and $\beta^{G}\left(A_{2}\left(f_{k}^{\epsilon,-}\right)\right)$. Since $\beta^{G}\left({ }^{0} A_{2}\left(f_{k}^{\epsilon, \eta}\right)\right)=u^{1+p+q} \beta^{G}\left(A_{1}^{0}\left(f_{k}^{\epsilon, \eta}\right)\right.$ ) (by proposition 6.3) and $A_{1}^{0}\left(f_{k}^{\epsilon, \eta}\right)=\mathcal{L}_{1}$, we are reduced to compare $\beta^{G}\left(A_{2}^{0}\left(f_{k}^{\epsilon,+}\right)\right)$ and $\beta^{G}\left(A_{2}^{0}\left(f_{k}^{\epsilon,-}\right)\right)$.

Denote $p$ the number of signs + and $q$ the number of signs - in the quadratic part of $f_{k}^{\epsilon,+}$ and $f_{k}^{\epsilon,-}$ (notice that $p q \neq 0$ ). Then, according to proposition 6.1,

$$
\beta^{G}\left(A_{2}^{0}\left(f_{k}^{\epsilon, \eta}\right)\right)=u^{2+p+q} \beta^{G}\left(Y_{p, q}\right) .
$$

Therefore, by proposition 5.1,

- if $p<q$, then

$$
\beta^{G}\left(A_{2}^{0}\left(f_{k}^{\epsilon,+}\right)\right)=u^{2+p+q} \frac{u^{p+q}-u^{q}+u^{p-1}}{u-1} \text { and } \beta^{G}\left(A_{2}^{0}\left(f_{k}^{\epsilon,-}\right)\right)=u^{2+p+q} \frac{u^{p+q}-u^{q}+u^{p+1}}{u-1},
$$

- if $q<p$, then

$$
\beta^{G}\left(A_{2}^{0}\left(f_{k}^{\epsilon,+}\right)\right)=u^{2+p+q} \frac{u^{p+q}-u^{p}+u^{q+1}}{u-1} \text { and } \beta^{G}\left(A_{2}^{0}\left(f_{k}^{\epsilon,-}\right)\right)=u^{2+p+q} \frac{u^{p+q}-u^{p}+u^{q-1}}{u-1}
$$

In particular, $\beta^{G}\left(A_{2}^{0}\left(f_{k}^{\epsilon,+}\right)\right) \neq \beta^{G}\left(A_{2}^{0}\left(f_{k}^{\epsilon,-}\right)\right)$ if $p \neq q$. Consequently, if $p \neq q$, the naive equivariant zeta functions of $f_{k}^{\epsilon,+}$ and $f_{k}^{\epsilon,-}$ are different and, by theorem 4.2 , these germs are not $G$-blow-Nash equivalent.

If $p=q, \beta^{G}\left(A_{2}^{0}\left(f_{k}^{\epsilon,+}\right)\right)=\beta^{G}\left(A_{2}^{0}\left(f_{k}^{\epsilon,-}\right)\right)$ and we look at the term $\beta^{G}\left(A_{2}^{+1}\left(f_{k}^{\epsilon, \eta}\right)\right)$ of the equivariant zeta functions with sign + . According to proposition 6.4,

$$
\beta^{G}\left(A_{2}^{+1}\left(f_{k}^{\epsilon, \eta}\right)\right)=u^{2+2 p} \beta^{G}\left(Y_{p, p}^{+1}\right)
$$

and, by $5.3, \beta^{G}\left(Y_{p, p}^{+1}\right)=\frac{1}{u-1}\left(\beta^{G}\left(Y_{p, p+1}\right)-\beta^{G}\left(Y_{p, p}\right)\right)$. Since the quantity $\beta^{G}\left(Y_{p, p}\right)$ is the same in either of the cases $\mathrm{n}^{\circ} 1$ and $\mathrm{n}^{\circ} 2$, we are reduced to compare the quantities $\beta^{G}\left(Y_{p, p+1}\right)$ in the cases $\mathrm{n}^{\circ} 1$ and $\mathrm{n}^{\mathrm{O}} 2$.

We have

$$
\beta^{G}\left(Y_{p, p+1}\right)=\left\{\begin{array}{l}
\frac{u^{2 p+1}-u^{p+1}+u^{p-1}}{u-1} \text { in the case } \mathrm{n}^{\mathrm{o}} 1, \\
\frac{u^{2 p+1}-u^{p+1}+u^{p+1}}{u-1} \text { in the case } \mathrm{n}^{\mathrm{o}} 2,
\end{array}\right.
$$


and, as a consequence, $\beta^{G}\left(A_{2}^{+1}\left(f_{k}^{\epsilon,+}\right)\right) \neq \beta^{G}\left(A_{2}^{+1}\left(f_{k}^{\epsilon,-}\right)\right)$, so $f_{k}^{\epsilon,+}$ and $f_{k}^{\epsilon,-}$ are not $G$-blow-Nash equivalent in the case $p=q$ as well.

If $k=1$, notice that $f_{1}^{\epsilon, \eta}(x, y)=\epsilon x^{2}+Q_{p, q}(y)$ and we are reduced to compare $\beta^{G}\left(A_{2}^{0}\left(f_{1}^{\epsilon,+}\right)\right)$ and $\beta^{G}\left(A_{2}^{0}\left(f_{1}^{\epsilon,-}\right)\right)$ as well. We have $\beta^{G}\left(A_{2}^{0}\left(f_{1}^{\epsilon, \eta}\right)\right)=u^{1+p+q} \beta^{G}\left(Y_{p+1, q}\right)$ if $\epsilon=+1$, and $\beta^{G}\left(A_{2}^{0}\left(f_{1}^{\epsilon, \eta}\right)\right)=$ $u^{1+p+q} \beta^{G}\left(Y_{p, q+1}\right)$ if $\epsilon=-1$. As above, we can show, for instance if $\epsilon=+1$, that $\beta^{G}\left(A_{2}^{0}\left(f_{1}^{\epsilon,+}\right)\right) \neq$ $\beta^{G}\left(A_{2}^{0}\left(f_{1}^{\epsilon,-}\right)\right)$ when $p+1 \neq q$, and $\beta^{G}\left(A_{2}^{+1}\left(f_{1}^{\epsilon,+}\right)\right) \neq \beta^{G}\left(A_{2}^{+1}\left(f_{1}^{\epsilon,-}\right)\right)$ if $p+1=q$.

Remark 6.7. If $k=0, f_{0}^{\epsilon, \eta}(x, y)=\epsilon x+Q_{p, q}(y)$ and, using the notations of the proof of proposition 6.1 , the left members of all the equations describing $A_{m}^{0}\left(f_{0}^{\epsilon, \eta}\right)$, resp. $A_{m}^{\xi}\left(f_{0}^{\epsilon, \eta}\right)$, for $m \geq 1$, contain a term $\epsilon a_{i}+\ldots$, so that each of these sets is equivariantly isomorphic to an affine space. As a consequence (see remark 4.1), the respective equivariant zeta functions of $f_{0}^{\epsilon,+}$ and $f_{0}^{\epsilon,-}$ are equal.

Corollary 6.8. Let $k \geq 2$. Suppose that the invariant germs

$$
f_{2 k-1}=\epsilon x_{2}^{2 k}+\eta x_{1}^{2}+Q \text { and } g_{k}=\epsilon x_{1}^{2 k}+\eta^{\prime} x_{2}^{2}+Q^{\prime}
$$

have, up to permutation of all variables, the same quadratic part, with $p$ signs + and $q$ signs - .

If $p \leq q$ and $\eta=+1$ or $q \leq p$ and $\eta=-1$, then $f_{2 k-1}$ and $g_{k}$ are not G-blow-Nash equivalent.

If $p=q+1$ or $q=p+1$, then $f_{2 k-1}$ and $g_{k}$ are not $G$-blow-Nash equivalent.

Proof. We first deal with the case $p \leq q$ and $\eta=+1$ (notice that $p \neq 0$ ) ; the case $q \leq p$ and $\eta=-1$ is symmetric.

As in the proof of previous corollary 6.6 , we have

$$
\beta^{G}\left(A_{2}^{0}\left(f_{2 k-1}\right)\right)=u^{2+p+q} \beta^{G}\left(Y_{p, q}\right) \text { and } \beta^{G}\left(A_{2}^{0}\left(g_{k}\right)\right)=u^{2+p+q} \beta^{G}\left(Y_{p, q}\right)
$$

where, in the left equality, the set $Y_{p, q}$ is equipped with the action $\mathrm{n}^{\circ} 1$ and, in the right one, with the trivial action of $G$. Since the corresponding equivariant virtual Poincaré series are different by proposition $5.1, \beta^{G}\left(A_{2}\left(f_{2 k-1}\right)\right) \neq \beta^{G}\left(A_{2}\left(g_{k}\right)\right)$ and the naive equivariant zeta functions of $f_{2 k-1}$ and $g_{k}$ are different. As a consequence, $f_{2 k-1}$ and $g_{k}$ are not $G$-blow-Nash equivalent.

Now we suppose $p=q+1$ (the case $q=p+1$ is symmetric). In particular $q<p$, so we can assume $\eta=+1$.

We consider $\beta^{G}\left(A_{2}^{+1}\left(f_{2 k-1}\right)\right)=u^{2+p+q} \beta^{G}\left(Y_{p, q}^{+1}\right)$ and $\beta^{G}\left(A_{2}^{+1}\left(g_{k}\right)\right)=u^{2+p+q} \beta^{G}\left(Y_{p, q}^{+1}\right)$ (proposition 6.4). Thanks to proposition 5.3, we know that $\beta^{G}\left(Y_{p, q}^{+1}\right)=\frac{1}{u-1}\left(\beta^{G}\left(Y_{p, q+1}\right)-\beta^{G}\left(Y_{p, q}\right)\right)$. By proposition 5.1, the respective quantities $\beta^{G}\left(Y_{p, q}\right)$ for $f_{2 k-1}$ and $g_{k}$ are equal, whereas the quantities $\beta^{G}\left(Y_{p, q+1}\right)=\beta^{G}\left(Y_{p, p}\right)$ are different. Consequently, the equivariant zeta functions with sign + of $f_{2 k-1}$ and $g_{k}$ are different and therefore the latter germs are not $G$-blow-Nash equivalent.

Remark 6.9. In the other cases, the quantities $\beta^{G}\left(Y_{p, q}\right)$ and $\beta^{G}\left(Y_{p, q}^{\xi}\right)$ are the same for $f_{2 k-1}$ and $g_{k}$. 


\subsection{Computation of $\beta^{G}\left(A_{2 k}\left(f_{2 k-1}\right)\right)$ and $\beta^{G}\left(A_{2 k}\left(g_{k}\right)\right)$}

For the continuation of the section, thanks to corollaries 6.6 and 6.8 , we only need to consider the germs

$$
f_{2 k-1}=\epsilon x_{2}^{2 k}+\eta x_{1}^{2}+Q \text { and } g_{k}=\epsilon x_{1}^{2 k}+\eta^{\prime} x_{2}^{2}+Q^{\prime}
$$

assumed to have the same quadratic part $Q_{p, q}$, such that $p>q+1$ and $\eta=+1$ or $q>p+1$ and $\eta=-1$.

In order to prove that the germs $f_{2 k-1}$ and $g_{k}$ are not $G$-blow-Nash equivalent in some of these cases as well, we will compute the coefficients $\beta^{G}\left(A_{2 k}\left(f_{2 k-1}\right)\right)$ and $\beta^{G}\left(A_{2 k}\left(g_{k}\right)\right)$ of their respective naive equivariant zeta functions :

Proposition 6.10. Suppose $k \geq 2$.

1. If $p q=0$, then

$$
\beta^{G}\left(A_{2 k}^{0}\left(f_{2 k-1}\right)\right)=u^{2 k-1+k(p+q)} \beta^{G}\left(\left\{f_{2 k-1}=0\right\}\right) \text { and } \beta^{G}\left(A_{2 k}^{0}\left(g_{k}\right)\right)=u^{2 k-1+k(p+q)} \beta^{G}\left(\left\{g_{k}=0\right\}\right) .
$$

2. If $p q \neq 0$, then

$$
\beta^{G}\left(A_{2 k}^{0}\left(f_{2 k-1}\right)\right)=u^{2 k-2} u^{(p+q)(k+1)} \beta^{G}\left(Y_{p, q} \backslash\{0\}\right) \frac{u^{(p+q-2)(k-1)}-1}{u^{p+q-2}-1}+u^{k(p+q)+2 k-1} \beta^{G}\left(\left\{f_{2 k-1}=0\right\}\right)
$$

(the group $G$ acts on $Y_{p, q}$ via the involution $n^{\circ} 1$ or $n^{\circ} 2$ depending on the sign of $\eta$ ) and

$$
\beta^{G}\left(A_{2 k}^{0}\left(g_{k}\right)\right)=u^{2 k-2} u^{(p+q)(k+1)} \beta^{G}\left(Y_{p, q} \backslash\{0\}\right) \frac{u^{(p+q-2)(k-1)}-1}{u^{p+q-2}-1}+u^{k(p+q)+2 k-1} \beta^{G}\left(\left\{g_{k}=0\right\}\right)
$$

(the group $G$ acts trivially on $Y_{p, q}$ ).

Proof. We keep the notations of the proof of proposition 6.1 and we proceed as in [13] Proof of Lemma 3.3. First suppose that $p q \neq 0$. An $\operatorname{arc} \gamma$ of $\mathcal{L}_{2 k}$ belongs to $A_{2 k}^{0}\left(f_{2 k-1}\right)$ if and only if

$$
\left\{\begin{array}{l}
Q_{p, q}\left(c_{1}\right)=0, \\
\Phi_{p, q}\left(c_{1}, c_{2}\right)=0, \\
\cdots \\
\sum_{t=1}^{k-1} \Phi_{p, q}\left(c_{t}, c_{2 k-1-t}\right)=0, \\
\epsilon a_{1}^{2 k}+Q_{p, q}\left(c_{k}\right)+\sum_{t=1}^{k-1} \Phi_{p, q}\left(c_{t}, c_{2 k-t}\right)=0 .
\end{array}\right.
$$

We have

$$
\beta^{G}\left(A_{2 k}^{0}\left(f_{2 k-1}\right)\right)=u^{2 k+(2 k-1)(p+q-1)+1} \beta^{G}\left(Y_{p, q} \backslash\{0\}\right)+\beta^{G}\left(A_{2 k}^{0}\left(f_{2 k-1}\right) \cap\left\{c_{1}^{1}=\ldots=c_{1}^{p}=0\right\}\right),
$$

and $\beta^{G}\left(A_{2 k}^{0}\left(f_{2 k-1}\right) \cap\left\{c_{1}^{1}=\ldots=c_{1}^{p}=0\right\}\right)=u^{2+p+q} \beta^{G}\left(C_{2 k-2}^{0}\right)$, if $C_{2 k-2}^{0}$ denotes the algebraic set described by the equations

$$
\left\{\begin{array}{l}
Q_{p, q}\left(c_{1}\right)=0, \\
\Phi_{p, q}\left(c_{1}, c_{2}\right)=0, \\
\cdots \\
\sum_{t=1}^{k-2} \Phi_{p, q}\left(c_{t}, c_{2 k-3-t}\right)=0 \\
\epsilon a_{1}^{2 k}+Q_{p, q}\left(c_{k-1}\right)+\sum_{t=1}^{k-2} \Phi_{p, q}\left(c_{t}, c_{2 k-2-t}\right)=0 .
\end{array}\right.
$$


By an induction, we obtain

$$
\beta^{G}\left(A_{2 k}^{0}\left(f_{2 k-1}\right)\right)=\beta^{G}\left(Y_{p, q} \backslash\{0\}\right) \sum_{t=0}^{k-2} u^{t(2+p+q)} u^{2 k-2 t+(2 k-2 t-1)(p+q-1)+1}+u^{(k-1)(2+p+q)} \beta^{G}\left(C_{2}^{0}\right) .
$$

Since $C_{2}^{0}$ is defined by the equation $\epsilon a_{1}^{2 k}+Q_{p, q}\left(c_{1}\right)=0$ and since the vector $c_{2}$ and the variable $a_{2}$ are free, we deduce the desired expression for $\beta^{G}\left(A_{2 k}^{0}\left(f_{2 k-1}\right)\right)$. The steps of computation are the same for $\beta^{G}\left(A_{2 k}^{0}\left(g_{k}\right)\right)$.

If $p q=0$, the vectors $c_{1}, \ldots, c_{k-1}$ are zero vectors and the system is reduced to the equation $\epsilon a_{1}^{2 k}+Q_{p, q}\left(c_{k}\right)=0$, the other variables being free.

Since $p>q+1$ and $\eta=+1$ or $q>p+1$ and $\eta=-1$, the quantity $\beta^{G}\left(Y_{p, q}\right)$ is the same for $f_{2 k-1}$ and $g_{k}$. As a consequence, in order to compare $\beta^{G}\left(A_{2 k}\left(f_{2 k-1}\right)\right)$ and $\beta^{G}\left(A_{2 k}\left(g_{k}\right)\right)$, we are reduced to consider the quantities $\beta^{G}\left(\left\{f_{2 k-1}=0\right\}\right)$ and $\beta^{G}\left(\left\{g_{k}=0\right\}\right)$ (notice that $\beta^{G}\left({ }^{0} A_{2 k}\left(f_{2 k-1}\right)\right)=\beta^{G}\left({ }^{0} A_{2 k}\left(g_{k}\right)\right)$ by the results of the previous paragraph 6.1). We compute these equivariant virtual Poincaré series for all $k \geq 2, p, q \in \mathbb{N}$ and $\eta \in\{1,-1\}$ :

Lemma 6.11. We have

$$
\beta^{G}\left(\left\{f_{2 k-1}=0\right\}\right)=\beta^{G}\left(\left\{\epsilon x_{2}^{2}+\eta x_{1}^{2}+Q=0\right\}\right)-(k-1) \beta^{G}\left(\left\{\eta x_{1}^{2}+Q=0\right\}\right)+(k-1) \beta^{G}(\{0\}),
$$

where the second set in the right member is considered as an algebraic subset of $\mathbb{R}^{n-1}$ and $G$ acts on the considered sets via the involution $n^{\circ} 1$ or $n^{\circ} 2$ depending on the sign of $\eta$, and

$\beta^{G}\left(\left\{g_{k}=0\right\}\right)=\beta^{G}\left(\left\{\epsilon x_{1}^{2}+\eta^{\prime} x_{2}^{2}+Q^{\prime}=0\right\}\right)-\rho \beta^{G}\left(\left\{\eta^{\prime} x_{2}^{2}+Q^{\prime}=0\right\}\right)-\tau \beta^{G}\left(\left\{\eta^{\prime} x_{2}^{2}+Q^{\prime}=0\right\}\right)+(k-1) \beta^{G}(\{0\})$,

where the second and third sets in the right member are considered as algebraic subsets of $\mathbb{R}^{n-1}$, the group $G$ acts on the second set via the involution $n^{\circ} 4$ (trivial action), on the third set via the involution $n^{\circ} 3$ (change of signs of all coordinates) and

1. if $k=2 l+1$ is odd, then $\rho=\tau=l$ and $G$ acts on the first set in the right member via the involution $n^{\circ} 1$ or $n^{\circ} 2$ depending on the sign of $\epsilon$,

2. if $k=2 l$ is even, then $\rho=l, \tau=l-1$ and $G$ acts on the first set in the right member via the involution $n^{\circ} 3$.

Proof. We begin with $\beta^{G}\left(\left\{f_{2 k-1}=0\right\}\right)$. Recall that $f_{2 k-1}\left(x_{1}, x_{2}, x_{3}, \ldots, x_{n}\right)=\epsilon x_{2}^{2 k}+\eta x_{1}^{2}+$ $Q\left(x_{3}, \ldots, x_{n}\right)$. We proceed to an equivariant blowing-up of the algebraic set $\left\{f_{2 k-1}=0\right\}$ at the origin of $\mathbb{R}^{n}$. In the chart $x_{2}=u, x_{i}=u v_{i}, i=1,3, \ldots, n$, the blown-up variety is defined by the equation

$$
u^{2} f_{2 k-3}\left(v_{1}, u, v_{3}, \ldots, v_{n}\right)=0,
$$

the action of $G$ being given by the involution $\left(v_{1}, u, v_{3}, \ldots, v_{n}\right) \mapsto\left(-v_{1}, u, v_{3}, \ldots, v_{n}\right)$. We have $\beta^{G}\left(\left\{f_{2 k-1}=0\right\} \backslash\{0\}\right)=\beta^{G}\left(\left\{f_{2 k-3}=0\right\} \backslash\{u=0\}\right)$, therefore

$$
\beta^{G}\left(\left\{f_{2 k-1}=0\right\}\right)=\beta^{G}\left(\left\{f_{2 k-3}=0\right\}\right)-\beta^{G}\left(\left\{\eta v_{1}^{2}+Q\left(v_{3}, \ldots, v_{n}\right)=0, u=0\right\}\right)+\beta^{G}(\{0\}) .
$$


We then obtain the desired result by an induction.

For the computation of $\beta^{G}\left(\left\{g_{k}=0\right\}\right)$, recall that $g_{k}\left(x_{1}, x_{2}, x_{3}, \ldots, x_{n}\right)=\epsilon x_{1}^{2 k}+\eta^{\prime} x_{2}^{2}+$ $Q^{\prime}\left(x_{3}, \ldots, x_{n}\right)$ and proceed to an equivariant blowing-up of the set $\left\{g_{k}=0\right\}$ at the origin of $\mathbb{R}^{n}$, looked at in the chart $x_{1}=u, x_{i}=u v_{i}, i=2,3, \ldots, n$. In this chart, the blown-up variety is defined by

$$
u^{2} g_{k-1}\left(u, v_{2}, v_{3}, \ldots, v_{n}\right)=0,
$$

the action of $G$ being given by the involution $\left(u, v_{2}, v_{3}, \ldots, v_{n}\right) \mapsto\left(-u,-v_{2},-v_{3}, \ldots,-v_{n}\right)$, and we have

$$
\left.\beta^{G}\left(\left\{g_{k}=0\right\}\right)=\beta^{G}\left(\left\{g_{k-1}=0\right\}\right)-\beta^{G}\left\{\eta^{\prime} x_{2}^{2}+Q^{\prime}\left(x_{3}, \ldots, x_{n}\right)=0\right\}\right)+\beta^{G}(\{0\}) .
$$

One further equivariant blowing-up of $\left\{g_{k-1}=0\right\}$ provides the equation

$$
u^{2} g_{k-1}\left(u, v_{2}, v_{3}, \ldots, v_{n}\right)=0,
$$

the group $G$ acting via the involution $\left(u, v_{2}, v_{3}, \ldots, v_{n}\right) \mapsto\left(-u, v_{2}, v_{3}, \ldots, v_{n}\right)$. The desired expression is then obtained by an induction.

Remark 6.12. According to proposition 5.1, the quantity $\beta^{G}\left(\left\{\eta^{\prime} x_{2}^{2}+Q^{\prime}=0\right\}\right)$ is the same if $G$ acts via the involution $\mathrm{n}^{\circ} 4$ or via the involution $\mathrm{n}^{\circ} 3$. Therefore, in the previous lemma 6.11 , we can simply write $\rho \beta^{G}\left(\left\{\eta^{\prime} x_{2}^{2}+Q^{\prime}=0\right\}\right)+\tau \beta^{G}\left(\left\{\eta^{\prime} x_{2}^{2}+Q^{\prime}=0\right\}\right)$ as $(k-1) \beta^{G}\left(\left\{\eta^{\prime} x_{2}^{2}+Q^{\prime}=0\right\}\right)$ with $G$ acting trivially on the latter set.

Because $p>q+1$ and $\eta=+1$ or $q>p+1$ and $\eta=-1$, we have $\beta^{G}\left(\left\{\eta x_{1}^{2}+Q=0\right\}\right)=$ $\beta^{G}\left(\left\{\eta^{\prime} x_{2}^{2}+Q^{\prime}=0\right\}\right)$ and we are finally reduced to compare $\beta^{G}\left(\left\{\epsilon x_{2}^{2}+\eta x_{1}^{2}+Q=0\right\}\right)$ and $\beta^{G}\left(\left\{\epsilon x_{1}^{2}+\eta^{\prime} x_{2}^{2}+Q^{\prime}=0\right\}\right)$. The cases where these quantities are different are cases where the germs $f_{2 k-1}$ and $g_{k}$ are not $G$-blow-Nash-equivalent :

Corollary 6.13. If $k$ is odd and if $p>q+1, \eta=+1$ and $\epsilon=-1$ or $q>p+1, \eta=-1$ and $\epsilon=+1$, then the germs $f_{2 k-1}$ and $g_{k}$ are not $G$-blow-Nash-equivalent.

Proof. Assume that $k$ is odd and suppose that $p>q+1, \eta=+1$ and $\epsilon=-1$ (the case $q>p+1, \eta=-1$ and $\epsilon=+1$ is symmetric). We have $\left\{\epsilon x_{2}^{2}+\eta x_{1}^{2}+Q=0\right\}=Y_{p, q+1}$, where $Y_{p, q+1}$ is equipped with the involution $\mathrm{n}^{\circ}$, and $\left\{\epsilon x_{1}^{2}+\eta^{\prime} x_{2}^{2}+Q^{\prime}=0\right\}=Y_{p, q+1}$, where $Y_{p, q+1}$ is equipped with the involution $\mathrm{n}^{\circ} 2$. Then, by proposition 5.1, $\beta^{G}\left(\left\{\epsilon x_{2}^{2}+\eta x_{1}^{2}+Q=0\right\}\right) \neq$ $\beta^{G}\left(\left\{\epsilon x_{1}^{2}+\eta^{\prime} x_{2}^{2}+Q^{\prime}=0\right\}\right)$ and $\beta^{G}\left(A_{2 k}\left(f_{2 k-1}\right)\right) \neq \beta^{G}\left(A_{2 k}\left(g_{k}\right)\right)$.

In the remaining cases, the quantities $\beta^{G}\left(\left\{\epsilon x_{2}^{2}+\eta x_{1}^{2}+Q=0\right\}\right)$ and $\beta^{G}\left(\left\{\epsilon x_{1}^{2}+\eta^{\prime} x_{2}^{2}+Q^{\prime}=0\right\}\right)$ are equal so that $\beta^{G}\left(A_{2 k}\left(f_{2 k-1}\right)\right)=\beta^{G}\left(A_{2 k}\left(g_{k}\right)\right)$. As a consequence, for these cases, we are led to look at the remaining coefficients of the equivariant zeta functions of $f_{2 k-1}$ and $g_{k}$. We begin, in the following paragraph, with the computation of the terms $\beta^{G}\left(A_{2 k}^{\xi}\left(f_{2 k-1}\right)\right)$ and $\beta^{G}\left(A_{2 k}^{\xi}\left(g_{k}\right)\right)$. 


\subsection{Computation of $\beta^{G}\left(A_{2 k}^{\xi}\left(f_{2 k-1}\right)\right)$ and $\beta^{G}\left(A_{2 k}^{\xi}\left(g_{k}\right)\right)$}

We assume we are not in one of the previous cases for which we showed that $f_{2 k-1}$ and $g_{k}$ are not $G$-blow-Nash-equivalent. In particular, we have $\beta^{G}\left(A_{m}\left(f_{2 k-1}\right)\right)=\beta^{G}\left(A_{m}\left(g_{k}\right)\right)$ and $\beta^{G}\left(A_{m}^{\xi}\left(f_{2 k-1}\right)\right)=\beta^{G}\left(A_{m}^{\xi}\left(g_{k}\right)\right)$ for $m<2 k$, and $\beta^{G}\left(A_{2 k}\left(f_{2 k-1}\right)\right)=\beta^{G}\left(A_{2 k}\left(g_{k}\right)\right)$.

Now, the same steps of computation as in the proof of proposition 6.10 provide the following formulae for $\beta^{G}\left(A_{2 k}^{\xi}\left(f_{2 k-1}\right)\right)$ and $\beta^{G}\left(A_{2 k}^{\xi}\left(g_{k}\right)\right)$ :

Proposition 6.14. Suppose $k \geq 2$.

1. If $p q=0$, then $\beta^{G}\left(A_{2 k}^{\xi}\left(f_{2 k-1}\right)\right)=u^{2 k-1+k(p+q)} \beta^{G}\left(\left\{f_{2 k-1}=\xi\right\}\right)$ and $\beta^{G}\left(A_{2 k}^{\xi}\left(g_{k}\right)\right)=u^{2 k-1+k(p+q)} \beta^{G}\left(\left\{g_{k}=\xi\right\}\right)$.

2. If $p q \neq 0$, then

$$
\beta^{G}\left(A_{2 k}^{\xi}\left(f_{2 k-1}\right)\right)=u^{2 k-2} u^{(p+q)(k+1)} \beta^{G}\left(Y_{p, q} \backslash\{0\}\right) \frac{u^{(p+q-2)(k-1)}-1}{u^{p+q-2}-1}+u^{k(p+q)+2 k-1} \beta^{G}\left(\left\{f_{2 k-1}=\xi\right\}\right)
$$

(the group $G$ acts on $Y_{p, q}$ via the involution $n^{\circ} 1$ or $n^{\circ} 2$ depending on the sign of $\eta$ ) and

$$
\beta^{G}\left(A_{2 k}^{\xi}\left(g_{k}\right)\right)=u^{2 k-2} u^{(p+q)(k+1)} \beta^{G}\left(Y_{p, q} \backslash\{0\}\right) \frac{u^{(p+q-2)(k-1)}-1}{u^{p+q-2}-1}+u^{k(p+q)+2 k-1} \beta^{G}\left(\left\{g_{k}=\xi\right\}\right)
$$

(the group $G$ acts trivially on $Y_{p, q}$ ).

As in the previous paragraph 6.2 , we are reduced to consider the quantities $\beta^{G}\left(\left\{f_{2 k-1}=\xi\right\}\right)$ and $\beta^{G}\left(\left\{g_{k}=\xi\right\}\right)$. We give below the first steps of computation of these equivariant virtual Poincaré series for all $k \geq 2,(p, q) \in \mathbb{N}^{2} \backslash\{(0,0)\}$ and $\eta \in\{1,-1\}$. We write $f_{2 k-1}=\epsilon x_{2}^{2 k}+$ $\eta x_{1}^{2}+Q=\epsilon x_{2}^{2 k}+\sum_{i=1}^{p} y_{i}^{2}-\sum_{j=1}^{q} y_{p+j}^{2}$ and $g_{k}=\epsilon x_{1}^{2 k}+\eta^{\prime} x_{2}^{2}+Q^{\prime}=\epsilon x_{1}^{2 k}+\sum_{i=1}^{p} y_{i}^{2}-\sum_{j=1}^{q} y_{p+j}^{2}$. Then :

Lemma 6.15. We have

$$
\beta^{G}\left(\left\{f_{2 k-1}=\xi\right\}\right)= \begin{cases}u^{q+2} \frac{u^{p-1}-1}{u-1}+u^{p-1} \beta^{G}\left(\left\{\epsilon x_{2}^{2 k}+y_{1}^{2}-y_{p+1}^{2}-\sum_{j=2 p+1}^{p+q} y_{j}^{2}=\xi\right\}\right) & \text { if } 0<p<q, \\ u^{p+2} \frac{u^{q-1}-1}{u-1}+u^{q-1} \beta^{G}\left(\left\{\epsilon x_{2}^{2 k}+y_{1}^{2}-y_{p+1}^{2}+\sum_{j=q+1}^{p} y_{j}^{2}=\xi\right\}\right) & \text { if } 0<q<p, \\ u^{p+2} \frac{u^{p-1}-1}{u-1}+u^{p-1} \beta^{G}\left(\left\{\epsilon x_{2}^{2 k}+y_{1}^{2}-y_{p+1}^{2}=\xi\right\}\right) & \text { if } p=q, \\ \beta^{G}\left(\left\{\epsilon x_{2}^{2 k}-y_{1}^{2}-\sum_{j=2}^{q} y_{j}^{2}=\xi\right\}\right) & \text { if } p=0, \\ \beta^{G}\left(\left\{\epsilon x_{2}^{2 k}+y_{1}^{2}+\sum_{i=2}^{p} y_{i}^{2}=\xi\right\}\right) & \text { if } q=0,\end{cases}
$$

the group $G$ acting only changing the sign of $y_{1}$ or $y_{p+1}$ depending on the sign of $\eta$, and

$$
\beta^{G}\left(\left\{g_{k}=\xi\right\}\right)= \begin{cases}u^{q+1} \frac{u^{p}-1}{u-1}+u^{p} \beta^{G}\left(\left\{\epsilon x_{1}^{2 k}-\sum_{j=2 p+1}^{p+q} y_{j}^{2}=\xi\right\}\right) & \text { if } 0<p<q, \\ u^{p+1} \frac{u^{q}-1}{u-1}+u^{q} \beta^{G}\left(\left\{\epsilon x_{1}^{2 k}+\sum_{j=q+1}^{p} y_{j}^{2}=\xi\right\}\right) & \text { if } 0<q<p, \\ u^{p+1} \frac{u^{p}-1}{u-1}+u^{p} \beta^{G}\left(\left\{\epsilon x_{1}^{2 k}=\xi\right\}\right) & \text { if } p=q, \\ \beta^{G}\left(\left\{\epsilon x_{1}^{2 k}-\sum_{j=1}^{q} y_{j}^{2}=\xi\right\}\right) & \text { if } p=0, \\ \beta^{G}\left(\left\{\epsilon x_{1}^{2 k}+\sum_{i=1}^{p} y_{i}^{2}=\xi\right\}\right) & \text { if } q=0,\end{cases}
$$

the group $G$ acting only changing the sign of $x_{1}$. 
Proof. We focus on the case $2 \leq p \leq q$ and proceed as in the proof of proposition 5.1: in order to compute $\beta^{G}\left(\left\{f_{2 k-1}=\xi\right\}\right)$, we apply the (equivariant) change of variables $u_{i}=y_{i}+y_{i+p}$, $v_{i}=y_{i}-y_{i+p}$ for $i=2, \ldots, p$ and the equation $f_{2 k-1}=\xi$ becomes

$$
\epsilon x_{2}^{2 k}+y_{1}^{2}-y_{p+1}^{2}+\sum_{i=2}^{p} u_{i} v_{i}-\sum_{j=2 p+1}^{p+q} y_{j}^{2}=\xi .
$$

Then, as in the proof of proposition 5.1, we use the stratification by the globally $G$-stable subsets $\left\{f_{2 k-1}=\xi\right\} \cap\left\{u_{2}=\ldots=u_{i}=0, u_{i+1} \neq 0\right\}$, along with the additivity of the equivariant virtual Poincaré series, to obtain the desired formula for $\beta^{G}\left(\left\{f_{2 k-1}=\xi\right\}\right)$.

As for $\beta^{G}\left(\left\{g_{k}=\xi\right\}\right)$, we can apply the equivariant change of variables $u_{i}=y_{i}+y_{i+p}$, $v_{i}=y_{i}-y_{i+p}$ for $i=1, \ldots, p$ (the strata $\left\{g_{k}=\xi\right\} \cap\left\{u_{1}=\ldots=u_{i}=0, u_{i+1} \neq 0\right\}$ are $G$-globally stable).

Remark 6.16. Regarding the equation $f_{2 k-1}=\xi$, we could also have applied the change of variables $u_{1}=y_{1}+y_{p+1}, v_{1}=y_{1}-y_{p+1}$, provided $G$ acts on these new coordinates via the involution $\left(u_{1}, v_{1}\right) \mapsto\left(-v_{1},-u_{1}\right)$ or $\left(u_{1}, v_{1}\right) \mapsto\left(v_{1}, u_{1}\right)$ (depending on the sign of $\left.\eta\right)$. However, the stratum $\left\{f_{2 k-1}=\xi\right\} \cap\left\{u_{1} \neq 0\right\}$ is not globally stable under this action of $G$.

From these formulae, among the remaining cases for which we did not establish that the germs $f_{2 k-1}$ and $g_{k}$ are not $G$-blow-Nash equivalent, we first extract the cases for which $\beta^{G}\left(A_{2 k}^{\xi}\left(f_{2 k-1}\right)\right)=\beta^{G}\left(A_{2 k}^{\xi}\left(g_{k}\right)\right)$ :

Proposition 6.17. If $p>q+1$ and $\eta=\epsilon=+1$ or $q>p+1$ and $\eta=\epsilon=-1$, we have $\beta^{G}\left(A_{2 k}^{\xi}\left(f_{2 k-1}\right)\right)=\beta^{G}\left(A_{2 k}^{\xi}\left(g_{k}\right)\right)$.

Proof. Similarly to the previous proofs, we focus on the case $p>q+1, q \neq 0$ and $\eta=\epsilon=+1$. Then $\beta^{G}\left(\left\{f_{2 k-1}=\xi\right\}\right)=u^{p+2} \frac{u^{q-1}-1}{u-1}+u^{q-1} \beta^{G}\left(\left\{+x_{2}^{2 k}+y_{1}^{2}-y_{p+1}^{2}+\sum_{j=q+1}^{p} y_{j}^{2}=\xi\right\}\right)$. On the latter set, the action of $G$ only changes the sign of $y_{1}$, so that we can use the equivariant change of variables $u=y_{q+1}+y_{p+1}, v=y_{q+1}-y_{p+1}$ in order to obtain the equality

$$
\beta^{G}\left(\left\{f_{2 k-1}=\xi\right\}\right)=u^{p+1} \frac{u^{q}-1}{u-1}+u^{q} \beta^{G}\left(\left\{+x_{2}^{2 k}+y_{1}^{2}+\sum_{j=q+2}^{p} y_{j}^{2}=\xi\right\}\right) .
$$

Therefore $\beta^{G}\left(A_{2 k}^{\xi}\left(f_{2 k-1}\right)\right)=\beta^{G}\left(A_{2 k}^{\xi}\left(g_{k}\right)\right)$ if and only if $\beta^{G}\left(\left\{+x_{2}^{2 k}+y_{1}^{2}+\sum_{j=q+2}^{p} y_{j}^{2}=\xi\right\}\right)=$ $\beta^{G}\left(\left\{+x_{1}^{2 k}+\sum_{j=q+1}^{p} y_{j}^{2}=\xi\right\}\right)$ (recall that, on the latter set, the action of $G$ only changes the sign of $x_{1}$ ).

Now, if $\xi=-1$, both sets are empty and if $\xi=+1$, they are compact, nonsingular and equivariantly homeomorphic to spheres having a non-empty fixed point set. As a consequence, for $\xi= \pm 1, \beta^{G}\left(\left\{+x_{2}^{2 k}+y_{1}^{2}+\sum_{j=q+2}^{p} y_{j}^{2}=\xi\right\}\right)=\beta^{G}\left(\left\{+x_{1}^{2 k}+\sum_{j=q+1}^{p} y_{j}^{2}=\xi\right\}\right)$ (see re$\operatorname{mark} 4.1)$ and $\beta^{G}\left(A_{2 k}^{\xi}\left(f_{2 k-1}\right)\right)=\beta^{G}\left(A_{2 k}^{\xi}\left(g_{k}\right)\right)$. 
Finally, we give the cases for which the equality $\beta^{G}\left(A_{2 k}^{\xi}\left(f_{2 k-1}\right)\right)=\beta^{G}\left(A_{2 k}^{\xi}\left(g_{k}\right)\right)$ depends on the equality of two equivariant virtual Poincaré series :

\section{Proposition 6.18.}

- If $k$ is even and if $p>q+1, \eta=+1$ and $\epsilon=-1$, the equality $\beta^{G}\left(A_{2 k}^{\xi}\left(f_{2 k-1}\right)\right)=$ $\beta^{G}\left(A_{2 k}^{\xi}\left(g_{k}\right)\right)$ is true if and only if the equivariant virtual Poincaré series of the algebraic subsets $\left\{-x_{2}^{2 k}+y^{2}+\sum_{i=1}^{K-1} y_{i}^{2}=\xi\right\} \subset \mathbb{R}^{K+1}, K:=p-q$, equipped with the action of $G$ only changing the sign of $y$, and $\left\{-x_{1}^{2 k}+\sum_{i=1}^{K} z_{i}^{2}=\xi\right\} \subset \mathbb{R}^{K+1}$, equipped with the action of $G$ only changing the sign of $x_{1}$, are equal.

- If $k$ is even and if $q>p+1, \eta=-1$ and $\epsilon=+1$, we have $\beta^{G}\left(A_{2 k}^{\xi}\left(f_{2 k-1}\right)\right)=\beta^{G}\left(A_{2 k}^{\xi}\left(g_{k}\right)\right)$ if and only if $\beta^{G}\left(\left\{x_{2}^{2 k}-y^{2}-\sum_{i=1}^{K-1} y_{i}^{2}=\xi\right\}\right)=\beta^{G}\left(\left\{x_{1}^{2 k}-\sum_{i=1}^{K} z_{i}^{2}=\xi\right\}\right)$.

Proof. If we focus on the case $p>q+1, q \neq 0, \eta=+1$ and $\epsilon=-1$, the same computation as in the proof of the previous proposition 6.17 provides the equivalence $\beta^{G}\left(A_{2 k}^{\xi}\left(f_{2 k-1}\right)\right)=$ $\beta^{G}\left(A_{2 k}^{\xi}\left(g_{k}\right)\right)$ if and only if $\beta^{G}\left(\left\{-x_{2}^{2 k}+y_{1}^{2}+\sum_{j=q+2}^{p} y_{j}^{2}=\xi\right\}\right)=\beta^{G}\left(\left\{-x_{1}^{2 k}+\sum_{j=q+1}^{p} y_{j}^{2}=\xi\right\}\right)$.

Remark 6.19. 1. Recall that we showed in corollary 6.13 that the germs $f_{2 k-1}$ and $g_{k}$ are not $G$-blow-Nash equivalent in the case $k$ odd and $p>q+1, \eta=+1, \epsilon=-1$ or $q>p+1$, $\eta=-1, \epsilon=+1$ (notice that in the previous proof of proposition 6.18 , we did not use the fact that $k$ was even).

2. Forgetting the action of $G$, the virtual Poincaré polynomials of the algebraic subsets $\left\{x^{2 k}-\sum_{i=1}^{K} y_{i}^{2}=\xi\right\}, \xi= \pm 1$, of $\mathbb{R}^{K+1}$ can be computed using the invariance of the virtual Poincaré polynomial under bijection with $\mathcal{A S}$ graph (see [22]). However, we do not know if the equivariant virtual Poincaré series is invariant under equivariant bijection with $\mathcal{A S}$ graph.

As a consequence of the results of this subsection 6.3 , we will then consider the other coefficients $\beta^{G}\left(A_{M}\left(f_{2 k-1}\right)\right)$ and $\beta^{G}\left(A_{M}\left(g_{k}\right)\right)$, respectively $\beta^{G}\left(A_{M}^{\xi}\left(f_{2 k-1}\right)\right)$ and $\beta^{G}\left(A_{M}^{\xi}\left(g_{k}\right)\right)$, $M>2 k$, of the equivariant zeta functions of $f_{2 k-1}$ and $g_{k}$, in the cases of propositions 6.17 and 6.18. In the next paragraph, we will show that the comparison of these quantities reduces to the comparison of the equivariant virtual Poincaré series of $\left\{f_{2 k-1}=\xi\right\}$ and $\left\{g_{k}=\xi\right\}$ as well.

\subsection{The last terms of the equivariant zeta functions}

Suppose $p>q+1, \eta=\epsilon=+1$ or $k$ even, $p>q+1, \eta=+1, \epsilon=-1$. The following results will also be true for the respective symmetric cases.

We first establish the equality between the last coefficients of the naive equivariant zeta functions of $f_{2 k-1}$ and $g_{k}$ (and therefore the equality of $Z_{f_{2 k-1}}^{G}(u, T)$ and $Z_{g_{k}}^{G}(u, T)$ ) :

Proposition 6.20. For all $M>2 k$, we have $\beta^{G}\left(A_{M}\left(f_{2 k-1}\right)\right)=\beta^{G}\left(A_{M}\left(g_{k}\right)\right)$. 
Proof. Let $M$ be greater than $2 k$. We prove that $\beta^{G}\left(A_{M}^{0}\left(f_{2 k-1}\right)\right)=\beta^{G}\left(A_{M}^{0}\left(g_{k}\right)\right)$ (this will give the desired result because of proposition 6.3 and the additivity of the equivariant virtual Poincaré series).

As in the proofs of propositions 6.1 and 6.10 , consider the system of equations defining $A_{M}^{0}\left(f_{2 k-1}\right)$. The same computations will bring, in the expression of $\beta^{G}\left(A_{M}^{0}\left(f_{2 k-1}\right)\right)$, a contribution of (a multiple in $\mathbb{Z}[u]\left[\left[u^{-1}\right]\right]$ of $) \beta^{G}\left(Y_{p, q} \backslash\{0\}\right)$ and a contribution of the equivariant virtual Poincaré series of a set defined by a system whose first equation is $\epsilon a_{1}^{2 k}+Q_{p, q}\left(c_{1}\right)=0$. Stratifying this last algebraic set with the subsets $\left\{c_{1}^{1}=\ldots=c_{1}^{i-1}=0, c_{1}^{i} \neq 0\right\}, i=1, \ldots, p+q$, and $\left\{c_{1}=0\right\}$ provides a contribution of $\beta^{G}\left(\left\{f_{2 k-1}=0\right\} \backslash\{0\}\right)$ and a new system where $c_{1}=0, a_{1}=0$ and whose first (non trivial) equations are the ones defining $A_{m}^{0}\left(f_{2 k-1}\right)$ for $m=\min (M-2 k, 2 k)$.

As a consequence, we can repeat the same steps of computations on this system and this will give further contributions of $\beta^{G}\left(Y_{p, q} \backslash\{0\}\right)$ (provided by the equations $Q_{p, q}\left(c_{1}\right)=0$ ) and $\beta^{G}\left(\left\{f_{2 k-1}=0\right\} \backslash\{0\}\right)$ (provided by the equations $\left.\epsilon a_{j}^{2 k}+Q_{p, q}\left(c_{1}\right)=0\right)$.

Since these systems and these operations are also valid for the computation of $\beta^{G}\left(A_{M}^{0}\left(g_{k}\right)\right)$ and because, in the considered cases, the quantities $\beta^{G}\left(Y_{p, q}\right)$ are equal for $f_{2 k-1}$ and $g_{k}$ and $\beta^{G}\left(\left\{f_{2 k-1}=0\right\}\right)=\beta^{G}\left(\left\{g_{k}=0\right\}\right)$, the expressions of $\beta^{G}\left(A_{M}^{0}\left(f_{2 k-1}\right)\right)$ and $\beta^{G}\left(A_{M}^{0}\left(g_{k}\right)\right)$ are identical.

Similar considerations bring the following results for the last coefficients of the equivariant zeta functions with signs :

Proposition 6.21. 1. If $p>q+1, \eta=\epsilon=+1$, then, for all $M>2 k$, we have $\beta^{G}\left(A_{M}^{\xi}\left(f_{2 k-1}\right)\right)=$ $\beta^{G}\left(A_{M}^{\xi}\left(g_{k}\right)\right)$, and consequently $Z_{f_{2 k-1}}^{G, \pm}(u, T)=Z_{g_{k}}^{G, \pm}(u, T)$.

2. If $k$ is even and if $p>q+1, \eta=+1$ and $\epsilon=-1$, we have the equality $Z_{f_{2 k-1}}^{G, \xi}(u, T)=$ $Z_{g_{k}}^{G, \xi}(u, T)$ if and only if $\beta^{G}\left(\left\{-x_{2}^{2 k}+y^{2}+\sum_{i=1}^{K-1} y_{i}^{2}=\xi\right\}\right)=\beta^{G}\left(\left\{-x_{1}^{2 k}+\sum_{i=1}^{K} z_{i}^{2}=\xi\right\}\right)$ (the former set is a subset of $\mathbb{R}^{K+1}$ equipped with the action of $G$ only changing the sign of $y$ and the latter set is a subset of $\mathbb{R}^{K+1}$ equipped with the action of $G$ only changing the sign of $\left.x_{1}\right)$.

Proof. Let $M$ be greater than $2 k$. The system defining $A_{M}^{\xi}\left(f_{2 k-1}\right)$ is obtained by replacing 0 by $\xi$ in the right member of the last equation of the system defining $A_{M}^{0}\left(f_{2 k-1}\right)$. Consequently, the same arguments works as in the proof of previous proposition 6.20 and $\beta^{G}\left(A_{M}^{\xi}\left(f_{2 k-1}\right)\right)=$ $\beta^{G}\left(A_{M}^{\xi}\left(g_{k}\right)\right)$ if and only if the contribution given by the very last equation provided by the computation is the same for $f_{2 k-1}$ and $g_{k}$.

As in the proof of proposition 6.4, if $M$ is odd, this contribution is the equivariant virtual Poincaré series of an empty set, and if $M$ is even and not a multiple of $2 k$, it is $\beta^{G}\left(Y_{p, q}^{\xi}\right)$ : in both cases, $\beta^{G}\left(A_{M}^{\xi}\left(f_{2 k-1}\right)\right)=\beta^{G}\left(A_{M}^{\xi}\left(g_{k}\right)\right)$. Finally, if $M$ is a multiple of $2 k$, the respective contributions are $\beta^{G}\left(\left\{f_{2 k-1}=\xi\right\}\right)$ and $\beta^{G}\left(\left\{g_{k}=\xi\right\}\right)$, hence the result by lemma 6.15 (see also the proofs of propositions 6.17 and 6.18$)$. 


\subsection{Conclusion}

As a conclusion, we summarize and gather the results of the previous paragraphs in the following theorem :

Theorem 6.22. Let $k \geq 2$. Suppose that the invariant germs

$$
f_{2 k-1}=\epsilon x_{2}^{2 k}+\eta x_{1}^{2}+Q \text { and } g_{k}=\epsilon x_{1}^{2 k}+\eta^{\prime} x_{2}^{2}+Q^{\prime}
$$

have, up to permutation of all variables, the same quadratic part, with $p$ signs + and $q$ signs - .

1. If

- $p \leq q, \eta=+1$ or $q \leq p, \eta=-1$,

- $p=q+1$ or $q=p+1$,

- $k$ is odd and if $p>q+1, \eta=+1, \epsilon=-1$ or $q>p+1, \eta=-1, \epsilon=+1$,

then $f_{2 k-1}$ and $g_{k}$ are not $G$-blow-Nash equivalent.

2. If $p>q+1, \eta=\epsilon=+1$ or $q>p+1, \eta=\epsilon=-1$, then $Z_{f_{2 k-1}}^{G}(u, T)=Z_{g_{k}}^{G}(u, T)$ and $Z_{f_{2 k-1}}^{G, \xi}(u, T)=Z_{g_{k}}^{G, \xi}(u, T)$.

3. - If $k$ is even and if $p>q+1, \eta=+1, \epsilon=-1$, then $Z_{f_{2 k-1}}^{G}(u, T)=Z_{g_{k}}^{G}(u, T)$. Furthermore, $Z_{f_{2 k-1}}^{G, \xi}(u, T)=Z_{g_{k}}^{G, \xi}(u, T)$ if and only if $\beta^{G}\left(\left\{-x_{2}^{2 k}+y^{2}+\sum_{i=1}^{K-1} y_{i}^{2}=\xi\right\}\right)=$ $\beta^{G}\left(\left\{-x_{1}^{2 k}+\sum_{i=1}^{K} z_{i}^{2}=\xi\right\}\right)$.

- If $k$ is even and if $q>p+1, \eta=-1, \epsilon=+1$, then $Z_{f_{2 k-1}}^{G}(u, T)=Z_{g_{k}}^{G}(u, T)$. Furthermore, $Z_{f_{2 k-1}}^{G, \xi}(u, T)=Z_{g_{k}}^{G, \xi}(u, T)$ if and only if $\beta^{G}\left(\left\{x_{2}^{2 k}-y^{2}-\sum_{i=1}^{K-1} y_{i}^{2}=\xi\right\}\right)=$ $\beta^{G}\left(\left\{x_{1}^{2 k}-\sum_{i=1}^{K} z_{i}^{2}=\xi\right\}\right)$.

Remark 6.23. 1. As one can notice from the computations, the fact that the equivariant Poincaré series of a given sphere is the same for any action of $G$ on it with a non-empty fixed point set (see remark 4.1) induces equalities between coefficients of the respective equivariant zeta functions of $f_{2 k-1}$ and $g_{k}$.

2. If the equivariant virtual Poincaré series was proved to be an invariant under equivariant bijection with $\mathcal{A S}$ graph, this could allow to compute (and compare) the quantities $\beta^{G}\left(\left\{-x_{2}^{2 k}+y^{2}+\sum_{i=1}^{K-1} y_{i}^{2}=\xi\right\}\right)$ and $\beta^{G}\left(\left\{-x_{1}^{2 k}+\sum_{i=1}^{K} z_{i}^{2}=\xi\right\}\right)$.

\section{The germs $C_{k}$ and $D_{k}$}

In a second time, we plan to make progress towards the classification with respect to $G$-blowNash equivalence of the invariant germs of the families

$$
h_{k}^{\epsilon_{k}}(x):= \pm x_{1}^{2}+x_{2}^{2} x_{3}+\epsilon_{k} x_{3}^{k-1}+Q \quad \text { and } r_{k}^{\epsilon_{k}}(x):=x_{1}^{2} x_{2}+\epsilon_{k} x_{2}^{k}+ \pm x_{3}^{2}+Q^{\prime},
$$


where $\epsilon_{k} \in\{-1 ;+1\}$.

By the same arguments as in the introduction of section 6 , we know that if two germs $h_{k}^{\epsilon_{k}}$ and $h_{l}^{\epsilon_{l}}$ are $G$-blow-Nash equivalent, they have the same quadratic part up to permutation of the variables $x_{1}, x_{4}, \ldots, x_{n}$ and, by [13] Proposition 3.11, that $k=l$ and $\epsilon_{k}=\epsilon_{l}$. Therefore, inside the family $D_{k}$, it remains to show that the germs

$$
h_{k}^{\epsilon_{k},+}(x):=+x_{1}^{2}+x_{2}^{2} x_{3}+\epsilon_{k} x_{3}^{k-1}+Q \text { and } h_{k}^{\epsilon_{k},-}(x):=-x_{1}^{2}+x_{2}^{2} x_{3}+\epsilon_{k} x_{3}^{k-1}+Q^{\prime},
$$

where $\epsilon_{k} \in\{-1 ;+1\}$ and $+x_{1}^{2}+Q$ and $-x_{1}^{2}+Q^{\prime}$ are the same quadratic part up to permutation of the variables $x_{1}, x_{4}, \ldots, x_{n}$, are not $G$-blow-Nash equivalent.

As for the family $C_{k}$, if two germs $r_{k}^{\epsilon_{k}}$ and $r_{l}^{\epsilon_{l}}$ are $G$-blow-Nash equivalent, they have the same quadratic part up to permutation of the variables $x_{3}, \ldots, x_{n}, k=l$ and $\epsilon_{k}=\epsilon_{l}$.

On the other hand, if two germs $h_{k}^{\epsilon_{k}}$ and $r_{k^{\prime}}^{\epsilon_{k^{\prime}}}$ are $G$-blow-Nash equivalent then $k=k^{\prime}+1$, $\epsilon_{k}=\epsilon_{k^{\prime}}$ and $\pm x_{1}^{2}+Q$ and $\pm x_{2}^{2}+Q^{\prime}$ are the same quadratic part up to permutation of all variables. As a consequence, we focus on the comparison of the germs

$$
h_{k+1}=x_{2}^{2} x_{3}+\epsilon x_{3}^{k}+\eta x_{1}^{2}+Q \text { and } r_{k}=x_{1}^{2} x_{2}+\epsilon x_{2}^{k}+\eta^{\prime} x_{3}^{2}+Q^{\prime}
$$

where $\epsilon, \eta, \eta^{\prime} \in\{1,-1\}$ and $\eta x_{1}^{2}+Q=\eta^{\prime} x_{3}^{2}+Q^{\prime}$ up to permutation of all variables.

In the following, as we did for the families $A_{k}$ and $B_{k}$, we study and compare the respective equivariant zeta functions of $h_{k}$ and $r_{k}$ : using theorem 4.2, this allows to extract further cases of non-G-blow-Nash equivalence.

\subsection{Computation of the first terms of the equivariant zeta functions}

Fix $k \geq 4$ and consider the invariant germ $h_{k}^{\epsilon, \eta}\left(x_{1}, \ldots, x_{n}\right)=\eta x_{1}^{2}+x_{2}^{2} x_{3}+\epsilon x_{3}^{k-1}+Q$. Denote $x_{2}=x, x_{3}=z$ and $\eta x_{1}^{2}+Q=Q_{p, q}=\sum_{i=1}^{p} y_{i}^{2}-\sum_{j=1}^{q} y_{p+j}^{2}(G$ acts on the renamed coordinates via the involution $\mathrm{n}^{\circ} 1$ or $\mathrm{n}^{\circ} 2$ depending on the sign of $\left.\eta\right)$, so that $h_{k}^{\epsilon, \eta}(x, z, y)=x^{2} z+\epsilon z^{k-1}+$ $Q_{p, q}(y)$.

The following proposition gives the computed expressions for $\beta^{G}\left(A_{m}^{0}\left(h_{k}^{\epsilon, \eta}\right)\right)$ (see the beginning of paragraph 6.1 for the definition of $A_{m}^{0}(h)$ for $h$ an invariant Nash germ) if $m<k-1$. The same expressions can be obtained for $\beta^{G}\left(A_{m}^{0}\left(r_{k-1}^{\epsilon}\right)\right)$, providing $Y_{p, q}$ is equipped with the trivial action in this case.

Proposition 7.1. Suppose $m<k-1$.

1. If $p+q=1$, then

$$
\beta^{G}\left(A_{m}^{0}\left(h_{k}^{\epsilon, \eta}\right)\right)=\left\{\begin{array}{l}
r u^{2 m+1}+\frac{u^{4 r+4}}{u-1} \text { if } m=2 r+1, \\
(r-1) u^{2 m+1}+\frac{u^{4 r+2}}{u-1} \text { if } m=2 r .
\end{array}\right.
$$

2. If $p+q \neq 1$, then

$$
\beta^{G}\left(A_{m}^{0}\left(h_{k}^{\epsilon, \eta}\right)\right)=\left\{\begin{array}{l}
u^{3 r+2+(r+1)(p+q)} \frac{u^{r(p+q-1)}-1}{u^{p+q-1}-1}\left(\beta^{G}\left(Y_{p, q} \backslash\{0\}\right)+1\right)+\frac{u^{3 r+3+(r+1)(p+q)}}{u-1} \text { if } m=2 r+1, \\
u^{3 r+(r+1)(p+q)} \frac{u^{(r-1)(p+q-1)}-1}{u^{p+q-1}-1}\left(\beta^{G}\left(Y_{p, q} \backslash\{0\}\right)+1\right)+u^{3 r+1+r(p+q)} \beta^{G}\left(Y_{p, q}\right) \text { if } m=2 r .
\end{array}\right.
$$


Proof. As in subsection 6.1, we follow the computations of [13], paying attention to their equivariance with respect to the considered actions of $G$.

Here, we write an $\operatorname{arc} \gamma$ of $\mathcal{L}_{m}$ as

$$
\begin{aligned}
\gamma(t)= & \left(a_{1} t+\cdots+a_{m} t^{m}, b_{1} t+\cdots+b_{m} t^{m}, c_{1}^{1} t+\cdots+c_{m}^{1} t^{m}, \ldots, c_{1}^{p+q} t+\cdots+c_{m}^{p+q} t^{m}\right) \\
= & \left(\begin{array}{c}
a_{1} \\
b_{1} \\
c_{1}^{1} \\
\vdots \\
c_{1}^{p+q}
\end{array}\right) t+\cdots+\left(\begin{array}{c}
a_{m} \\
b_{m} \\
c_{m}^{1} \\
\vdots \\
c_{m}^{p+q}
\end{array}\right) t^{m}=\left(\begin{array}{c}
a_{1} \\
b_{1} \\
c_{1}
\end{array}\right) t+\cdots+\left(\begin{array}{c}
a_{m} \\
b_{1} \\
c_{m}
\end{array}\right) t^{m}
\end{aligned}
$$

(the group $G$ acts on $\mathcal{L}_{m}$ changing the sign of the variables $c_{i}^{1}$, resp. $c_{i}^{p+1}$, in the case $\mathrm{n}^{\mathrm{o}} 1$, resp. $\left.\mathrm{n}^{\mathrm{O}} 2\right)$.

We focus on the generic case $p q \neq 0, p+q \neq 1$. First suppose that $m$ is odd, $m=2 r+1$. Then an arc $\gamma$ of $\mathcal{L}_{m}$ belongs to $A_{m}^{0}\left(h_{k}^{\epsilon, \eta}\right)$ if and only if

$$
\left\{\begin{array}{l}
Q_{p, q}\left(c_{1}\right)=0 \\
a_{1}^{2} b_{1}+\Phi_{p, q}\left(c_{1}, c_{2}\right)=0 \\
a_{1}^{2} b_{2}+2 a_{1} a_{2} b_{1}+Q_{p, q}\left(c_{2}\right)+\Phi_{p, q}\left(c_{1}, c_{3}\right)=0, \\
\cdots \\
\sum_{t=1}^{r-1} a_{t}^{2} b_{2 r-2 t}+2 \sum_{t=1}^{r-1} a_{t} \sum_{\delta=t+1}^{2 r-(t+1)} a_{\delta} b_{2 r-\delta-t}+Q_{p, q}\left(c_{r}\right)+\sum_{t=1}^{r-1} \Phi_{p, q}\left(c_{t}, c_{2 r-t}\right)=0, \\
\sum_{t=1}^{r} a_{t}^{2} b_{2 r+1-2 t}+2 \sum_{t=1}^{r-1} a_{s} \sum_{\delta=t+1}^{2 r+1-(t+1)} a_{\delta} b_{2 r+1-\delta-t}+\sum_{t=1}^{r} \Phi_{p, q}\left(c_{t}, c_{2 r+1-t}\right)=0 .
\end{array}\right.
$$

Stratifying $A_{m}^{0}\left(h_{k}^{\epsilon, \eta}\right)$ with the $G$-globally invariant subsets $\left\{c_{1}^{1}=\ldots=c_{1}^{i-1}=0, c_{1}^{i} \neq 0\right\}$, $i=1, \ldots, p$, and $\left\{c_{1}^{1}=\ldots=c_{1}^{p}=0\right\}=\left\{c_{1}=0\right\}$, as we did in the proof of proposition 6.1 , we obtain, by additivity of the equivariant virtual Poincaré series,

$$
\beta^{G}\left(A_{m}^{0}\left(h_{k}^{\epsilon, \eta}\right)\right)=u^{2 \times(2 r+1)+2 r(p-1)+2 r q+1} \beta^{G}\left(Y_{p, q} \backslash\{0\}\right)+\beta^{G}\left(A_{m}^{0}\left(h_{k}^{\epsilon, \eta}\right) \cap\left\{c_{1}=0\right\}\right),
$$

the algebraic set $A_{m}^{0}\left(h_{k}^{\epsilon, \eta}\right) \cap\left\{c_{1}=0\right\}$ being described by the system

$$
\left\{\begin{array}{l}
a_{1}^{2} b_{1}=0 \\
a_{1}^{2} b_{2}+2 a_{1} a_{2} b_{1}+Q_{p, q}\left(c_{2}\right)=0 \\
\cdots \\
\sum_{t=1}^{r-1} a_{t}^{2} b_{2 r-2 t}+2 \sum_{t=1}^{r-1} a_{t} \sum_{\delta=t+1}^{2 r-(t+1)} a_{\delta} b_{2 r-\delta-t}+Q_{p, q}\left(c_{r}\right)+\sum_{t=2}^{r-1} \Phi_{p, q}\left(c_{t}, c_{2 r-t}\right)=0, \\
\sum_{t=1}^{r} a_{t}^{2} b_{2 r+1-2 t}+2 \sum_{t=1}^{r-1} a_{s} \sum_{\delta=t+1}^{2 r+1-(t+1)} a_{\delta} b_{2 r+1-\delta-t}+\sum_{t=2}^{r} \Phi_{p, q}\left(c_{t}, c_{2 r+1-t}\right)=0 .
\end{array}\right.
$$

Now, if $a_{1} \neq 0$, then $b_{1}=0$ and the coordinates $b_{2}, \ldots, b_{2 r-1}$ are determined by $a_{1}$ and the other variables (via an equivariant morphism), and thus

$$
\beta^{G}\left(A_{m}^{0}\left(h_{k}^{\epsilon, \eta}\right) \cap\left\{c_{1}=0\right\}\right)=(u-1) \frac{u^{[2 r+2+2 r(p+q)]+1}}{u-1}+\beta^{G}\left(A_{m}^{0}\left(h_{k}^{\epsilon, \eta}\right) \cap\left\{c_{1}=0, a_{1}=0\right\}\right) .
$$


If $c_{1}=0$ and $a_{1}=0$, the remaining coordinates verify the system

$$
\left\{\begin{array}{l}
Q_{p, q}\left(c_{2}\right)=0 \\
a_{2}^{2} b_{1}+\Phi_{p, q}\left(c_{2}, c_{3}\right)=0 \\
a_{2}^{2} b_{2}+2 a_{2} a_{3} b_{1}+Q_{p, q}^{\prime}\left(c_{3}\right)+\Phi_{p, q}\left(c_{2}, c_{4}\right)=0, \\
\cdots \\
\sum_{t=2}^{r-1} a_{t}^{2} b_{2 r-2 t}+2 \sum_{t=2}^{r-1} a_{t} \sum_{\delta=t+1}^{2 r-(t+1)} a_{\delta} b_{2 r-\delta-t}+Q_{p, q}\left(c_{r}\right)+\sum_{t=2}^{r-1} \Phi_{p, q}\left(c_{t}, c_{2 r-t}\right)=0, \\
\sum_{t=2}^{r} a_{t}^{2} b_{2 r+1-2 t}+2 \sum_{t=2}^{r-1} a_{s} \sum_{\delta=t+1}^{2 r+1-(t+1)} a_{\delta} b_{2 r+1-\delta-t}+\sum_{t=2}^{r} \Phi_{p, q}\left(c_{t}, c_{2 r+1-t}\right)=0 .
\end{array}\right.
$$

Notice that the vector $c_{m}$ as well as the variables $a_{m}, b_{m-1}$ and $b_{m}$ are free and that, if we rename the variables, these equations define the set $A_{m-2}^{0}\left(h_{k}^{\epsilon, \eta}\right)$, so that

$$
\beta^{G}\left(A_{m}^{0}\left(h_{k}^{\epsilon, \eta}\right) \cap\left\{c_{1}=0, a_{1}=0\right\}\right)=u^{3+p+q} \beta^{G}\left(A_{m-2}^{0}\left(h_{k}^{\epsilon, \eta}\right)\right) .
$$

By an induction process, we then obtain

$$
\begin{aligned}
\beta^{G}\left(A_{m}^{0}\left(h_{k}^{\epsilon, \eta}\right)\right)= & \beta^{G}\left(Y_{p, q} \backslash\{0\}\right)\left[\sum_{t=0}^{r-1} u^{t(3+p+q)} u^{2 \times(m-2 t)+(m-2 t-1)(p+q-1)+1}\right] \\
& +\left[\sum_{t=0}^{r-1} u^{t(3+p+q)} u^{(m-1-2 t)(p+q+1)+3}\right]+u^{(r-1)(3+p+q)} \beta^{G}\left(A_{3}^{0}\left(h_{k}^{\epsilon, \eta}\right) \cap\left\{c_{1}=0, a_{1}=0\right\}\right),
\end{aligned}
$$

the equations for $A_{3}^{0}\left(h_{k}^{\epsilon, \eta}\right) \cap\left\{c_{1}=0, a_{1}=0\right\}$ becoming trivial. As a consequence (notice that for all $t=0, \ldots, r-1,2 \times(m-2 t)+(m-2 t-1)(p+q-1)+1=(m-1-2 t)(p+q+1)+3)$,

$$
\beta^{G}\left(A_{m}^{0}\left(h_{k}^{\epsilon, \eta}\right)\right)=u^{3 r+2+(r+1)(p+q)} \frac{u^{r(p+q-1)}-1}{u^{p+q-1}-1}\left(\beta^{G}\left(Y_{p, q} \backslash\{0\}\right)+1\right)+\frac{u^{3 r+3+(r+1)(p+q)}}{u-1} .
$$

If $m$ is even, $m=2 r$, the system defining $A_{m}^{0}\left(h_{k}^{\epsilon, \eta}\right)$ is

$$
\left\{\begin{array}{l}
Q_{p, q}\left(c_{1}\right)=0, \\
a_{1}^{2} b_{1}+\Phi_{p, q}\left(c_{1}, c_{2}\right)=0, \\
\cdots \\
\sum_{t=1}^{r-1} a_{t}^{2} b_{2 r-1-2 t}+2 \sum_{t=1}^{r-2} a_{s} \sum_{\delta=t+1}^{2 r-1-(t+1)} a_{\delta} b_{2 r-1-\delta-t}+\sum_{t=1}^{r-1} \Phi_{p, q}\left(c_{t}, c_{2 r-1-t}\right)=0, \\
\sum_{t=1}^{r-1} a_{t}^{2} b_{2 r-2 t}+2 \sum_{t=1}^{r-1} a_{t} \sum_{\delta=t+1}^{2 r-t+1)} a_{\delta} b_{2 r-\delta-t}+Q_{p, q}\left(c_{r}\right)+\sum_{t=1}^{r-1} \Phi_{p, q}\left(c_{t}, c_{2 r-t}\right)=0,
\end{array}\right.
$$

and we have

$$
\begin{aligned}
\beta^{G}\left(A_{m}^{0}\left(h_{k}^{\epsilon, \eta}\right)\right)= & \beta^{G}\left(Y_{p, q} \backslash\{0\}\right)\left[\sum_{t=0}^{r-2} u^{t(3+p+q)} u^{2 \times(m-2 t)+(m-2 t-1)(p+q-1)+1}\right] \\
& +\left[\sum_{t=0}^{r-2} u^{t(3+p+q)} u^{(m-1-2 t)(p+q+1)+3}\right]+u^{(r-1)(3+p+q)} \beta^{G}\left(A_{2}^{0}\left(h_{k}^{\epsilon, \eta}\right)\right)
\end{aligned}
$$


Because $A_{2}^{0}\left(h_{k}^{\epsilon, \eta}\right)$ is described by the equation $Q_{p, q}\left(c_{1}\right)=0$ and since the vector $c_{2}$ as well as the variables $a_{1}, a_{2}, b_{1}$ and $b_{2}$ are free, we obtain

$$
\beta^{G}\left(A_{m}^{0}\left(h_{k}^{\epsilon, \eta}\right)\right)=u^{3 r+(r+1)(p+q)} \frac{u^{(r-1)(p+q-1)}-1}{u^{p+q-1}-1}\left(\beta^{G}\left(Y_{p, q} \backslash\{0\}\right)+1\right)+u^{3 r+1+r(p+q)} \beta^{G}\left(Y_{p, q}\right) .
$$

As for $\beta^{G}\left(A_{m}^{\xi}\left(h_{k}^{\epsilon, \eta}\right)\right)$, we have the following expressions if $m<k+1$ :

Proposition 7.2. Suppose $m<k-1$.

1. If $(p, q)=(0,1)$, then

$$
\beta^{G}\left(A_{m}^{\xi}\left(h_{k}^{\epsilon, \eta}\right)\right)=\left\{\begin{array}{l}
r u^{2 m+1} \text { if } m=2 r+1 \\
(r-1) u^{2 m+1}+u^{4 r+1} \beta^{G}\left(Y_{0,1}^{\xi}\right) \text { if } m=2 r .
\end{array}\right.
$$

2. If $(p, q)=(1,0)$, then

$$
\beta^{G}\left(A_{m}^{\xi}\left(h_{k}^{\epsilon, \eta}\right)\right)=\left\{\begin{array}{l}
r u^{2 m+1} \text { if } m=2 r+1 \\
(r-1) u^{2 m+1}+u^{4 r+1} \beta^{G}\left(Y_{1,0}^{\xi}\right) \text { if } m=2 r
\end{array}\right.
$$

3. If $p+q \neq 1$, then

$$
\beta^{G}\left(A_{m}^{\xi}\left(h_{k}^{\epsilon, \eta}\right)\right)=\left\{\begin{array}{l}
u^{3 r+2+(r+1)(p+q)} \frac{u^{r(p+q-1)}-1}{u^{p+q-1}-1}\left(\beta^{G}\left(Y_{p, q} \backslash\{0\}\right)+1\right) \text { if } m=2 r+1, \\
u^{3 r+(r+1)(p+q)} \frac{u^{(r-1)(p+q-1)}-1}{u^{p+q-1}-1}\left(\beta^{G}\left(Y_{p, q} \backslash\{0\}\right)+1\right)+u^{3 r+1+r(p+q)} \beta^{G}\left(Y_{p, q}^{\xi}\right) \text { if } m=2 r .
\end{array}\right.
$$

Proof. If we keep the notations of the proof of proposition 7.1, the system $\operatorname{defining} A_{m}^{\xi}\left(h_{k}^{\epsilon, \eta}\right)$ is obtained by replacing 0 by $\xi$ in the right member of the last of the equations describing $A_{m}^{0}\left(h_{k}^{\epsilon, \eta}\right)$. Furthermore, the system for $A_{3}^{\xi}\left(h_{k}^{\epsilon, \eta}\right) \cap\left\{c_{1}=0, a_{1}=0\right\}$ has no solution, whereas $A_{2}^{\xi}\left(h_{k}^{\epsilon, \eta}\right)$ is described by the equation $Q_{p, q}\left(c_{1}\right)=\xi$.

We are now able to show that the germs $h_{k}^{\epsilon,+}$ and $h_{k}^{\epsilon,-}$ are not $G$-blow-Nash equivalent :

Corollary 7.3. Let $k \geq 4$. Suppose that the invariant Nash germs

$$
h_{k}^{\epsilon,+}(x):=+x_{1}^{2}+x_{2}^{2} x_{3}+\epsilon x_{3}^{k-1}+Q \text { and } h_{k}^{\epsilon,-}(x):=-x_{1}^{2}+x_{2}^{2} x_{3}+\epsilon x_{3}^{k-1}+Q^{\prime}
$$

have the same quadratic part up to permutation of the variables $x_{1}, x_{4}, \ldots, x_{n}$. Then they are not $G$-blow-Nash equivalent.

Proof. We compare $\beta^{G}\left(A_{2}\left(h_{k}^{\epsilon,+}\right)\right)$ and $\beta^{G}\left(A_{2}\left(h_{k}^{\epsilon,-}\right)\right)$. Because $\beta^{G}\left(A_{2}\left(h_{k}^{\epsilon, \eta}\right)\right)=\beta^{G}\left({ }^{0} A_{2}\left(h_{k}^{\epsilon, \eta}\right)\right)-$ $\beta^{G}\left(A_{2}^{0}\left(h_{k}^{\epsilon, \eta}\right)\right), \beta^{G}\left({ }^{0} A_{2}\left(h_{k}^{\epsilon, \eta}\right)\right)=u^{n} \beta^{G}\left(A_{1}^{0}\left(h_{k}^{\epsilon, \eta}\right)\right)$ (by proposition 6.3) and $A_{1}^{0}\left(h_{k}^{\epsilon, \eta}\right)=\mathcal{L}_{1}$, we are reduced to compare the quantities $\beta^{G}\left(A_{2}^{0}\left(h_{k}^{\epsilon,+}\right)\right)$ and $\beta^{G}\left(A_{2}^{0}\left(h_{k}^{\epsilon,-}\right)\right)$. 
Now, if $p$ denotes the number of signs + and $q$ the number of signs - in the quadratic part of $h_{k}^{\epsilon,+}$ and $h_{k}^{\epsilon,-}$ (notice that $p+q \neq 1$ ), we have, by proposition 7.1,

$$
\left.\beta^{G}\left(A_{2}^{0}\left(h_{k}^{\epsilon, \eta}\right)\right)\right)=u^{4+p+q} \beta^{G}\left(Y_{p, q}\right)
$$

Consequently, if $p \neq q$, we can use the same arguments as in the proof of corollary 6.6 to conclude that the naive equivariant zeta functions of $h_{k}^{\epsilon,+}$ and $h_{k}^{\epsilon,-}$ are different and therefore that the germs $h_{k}^{\epsilon,+}$ and $h_{k}^{\epsilon,-}$ are not $G$-blow-Nash equivalent.

If $p=q$, we compare $\beta^{G}\left(A_{2}^{+1}\left(h_{k}^{\epsilon,+}\right)\right)$ and $\beta^{G}\left(A_{2}^{+1}\left(h_{k}^{\epsilon,-}\right)\right)$. Since, by proposition 7.2,

$$
\beta^{G}\left(A_{2}^{+1}\left(h_{k}^{\epsilon,+}\right)\right)=u^{4+2 p} \beta^{G}\left(Y_{p, q}^{+1}\right),
$$

we can in this case as well use the arguments of the proof of corollary 6.6 , in order to conclude that the equivariant zeta functions with signs + of $h_{k}^{\epsilon,+}$ and $h_{k}^{\epsilon,-}$ are different.

Using again the formulae of propositions 7.1 and 7.2 , we then extract cases for which the germs $h_{k+1}$ and $r_{k}$ are not $G$-blow-Nash equivalent :

Corollary 7.4. Let $k \geq 3$. Suppose that the invariant germs

$$
h_{k+1}=x_{2}^{2} x_{3}+\epsilon x_{3}^{k}+\eta x_{1}^{2}+Q \text { and } r_{k}=x_{1}^{2} x_{2}+\epsilon x_{2}^{k}+\eta^{\prime} x_{3}^{2}+Q^{\prime}
$$

have, up to permutation of all variables, the same quadratic part, with $p$ signs + and $q$ signs - .

If $p \leq q$ and $\eta=+1$ or $q \leq p$ and $\eta=-1$, then $h_{k+1}$ and $r_{k}$ are not $G$-blow-Nash equivalent.

If $p=q+1$ or $q=p+1$, then $h_{k+1}$ and $r_{k}$ are not $G$-blow-Nash equivalent.

Proof. For the first point, focus for instance on the case $p \leq q$ and $\eta=+1$. As in the proof of corollary 7.3 , we consider $\beta^{G}\left(A_{2}^{0}\left(h_{k+1}\right)\right)=u^{4+p+q} \beta^{G}\left(Y_{p, q}\right)$ and $\beta^{G}\left(A_{2}^{0}\left(r_{k}\right)\right)=u^{4+p+q} \beta^{G}\left(Y_{p, q}\right)$. Since the action of $G$ on the former set $Y_{p, q}$ is the action $\mathrm{n}^{\circ} 1$ and the action on the latter set $Y_{p, q}$ is the trivial action, we obtain $\beta^{G}\left(A_{2}\left(h_{k+1}\right)\right) \neq \beta^{G}\left(A_{2}\left(r_{k}\right)\right)$.

For the second point, assume for instance $p=q+1$. Suppose furthermore that $\eta=+1$ and consider the quantities $\beta^{G}\left(A_{2}^{+1}\left(h_{k+1}\right)\right)=u^{4+p+q} \beta^{G}\left(Y_{p, q}^{+1}\right)$ and $\beta^{G}\left(A_{2}^{+1}\left(r_{k}\right)\right)=u^{4+p+q} \beta^{G}\left(Y_{p, q}^{+1}\right)$. By proposition 5.3, $\beta^{G}\left(Y_{p, q}^{+1}\right)=\frac{1}{u-1}\left(\beta^{G}\left(Y_{p, p}\right)-\beta^{G}\left(Y_{p, q}\right)\right)$. Since $q<p$ and $\eta=+1$, the quantity $\beta^{G}\left(Y_{p, q}\right)$ is the same for $h_{k+1}$ and $r_{k}$, while the quantities $\beta^{G}\left(Y_{p, p}\right)$ are different (see proposition 5.1). As a consequence, $\beta^{G}\left(A_{2}^{+1}\left(h_{k+1}\right)\right) \neq \beta^{G}\left(A_{2}^{+1}\left(r_{k}\right)\right)$.

From now, we are going to study the other coefficients of the equivariant zeta functions of $h_{k+1}$ and $r_{k}$ in the remaining cases, that is if $p>q+1$ and $\eta=+1$ or $q>p+1$ and $\eta=-1$. Notice that, in these cases, the quantities $\beta^{G}\left(Y_{p, q}\right)$ and $\beta^{G}\left(Y_{p, q}^{\xi}\right)$ are identical for $h_{k+1}$ and $r_{k}$.

\subsection{Computation of $\beta^{G}\left(A_{k}\left(h_{k+1}\right)\right)$ and $\beta^{G}\left(A_{k}\left(r_{k}\right)\right)$}

Assuming that the Nash germs $h_{k+1}$ and $r_{k}$ have the same quadratic part $Q_{p, q}$, with $p>q+1$ and $\eta=+1$ or $q>p+1$ and $\eta=-1$, we first compute the coefficients $\beta^{G}\left(A_{k}\left(h_{k+1}\right)\right)$ and $\beta^{G}\left(A_{k}\left(r_{k}\right)\right)$ of their respective naive equivariant zeta functions. Having in mind proposition 6.3 , we actually give formulae for $\beta^{G}\left(A_{k}^{0}\left(h_{k+1}\right)\right)$ and $\beta^{G}\left(A_{k}^{0}\left(r_{k}\right)\right)$ : 
Proposition 7.5. Suppose $k \geq 3$. Then

$\beta^{G}\left(A_{k}^{0}\left(h_{k+1}\right)\right)=\left\{\begin{array}{l}u^{3 l+2+(l+1)(p+q)} \frac{u^{l(p+q-1)}-1}{u^{p+q-1}-1}\left(\beta^{G}\left(Y_{p, q} \backslash\{0\}\right)\right) \\ +u^{3 l+1+(l+2)(p+q)} \frac{u^{(l-1)(p+q-1)}-1}{u^{p+q-1}-1}+u^{3 l+1+(l+1)(p+q)} \beta^{G}\left(\left\{h_{k+1}\left(x_{2}, x_{3}, 0\right)=0\right\}\right) \text { if } k=2 l+1, \\ u^{3 l+(l+1)(p+q)} \frac{u^{(l-1)(p+q)}-1}{u^{p+q-1}-1}\left(\beta^{G}\left(Y_{p, q} \backslash\{0\}\right)+1\right) \\ \quad+u^{3 l+l(p+q)} \beta^{G}\left(\left\{h_{k+1}\left(0, x_{3}, y\right)=0\right\}\right) \text { if } k=2 l,\end{array}\right.$

and

$\beta^{G}\left(A_{k}^{0}\left(r_{k}\right)\right)=\left\{\begin{array}{c}u^{3 l+2+(l+1)(p+q)} \frac{u^{l(p+q-1)}-1}{u^{p+q-1}-1}\left(\beta^{G}\left(Y_{p, q} \backslash\{0\}\right)\right) \\ \quad+u^{3 l+1+(l+2)(p+q)} \frac{u^{(l-1)(p+q-1)}-1}{u^{p+q-1}-1}+u^{3 l+1+(l+1)(p+q)} \beta^{G}\left(\left\{r_{k}\left(x_{1}, x_{2}, 0\right)=0\right\}\right) \text { if } k=2 l+1, \\ u^{3 l+(l+1)(p+q)} \frac{u^{(l-1)(p+q-1)}-1}{u^{p+q-1}-1}\left(\beta^{G}\left(Y_{p, q} \backslash\{0\}\right)+1\right)+u^{3 l+l(p+q)} \beta^{G}\left(\left\{r_{k}\left(0, x_{2}, y\right)=0\right\}\right) \text { if } k=2 l .\end{array}\right.$

Proof. We do the computations for $\beta^{G}\left(A_{k}^{0}\left(h_{k+1}\right)\right)$.

First suppose $k$ to be odd, $k=2 l+1$. Keeping the notations of the proof of proposition

7.1 , the set $A_{k}^{0}\left(h_{k+1}\right)$ is defined by the system

$$
\left\{\begin{array}{l}
Q_{p, q}\left(c_{1}\right)=0, \\
a_{1}^{2} b_{1}+\Phi_{p, q}\left(c_{1}, c_{2}\right)=0, \\
a_{1}^{2} b_{2}+2 a_{1} a_{2} b_{1}+Q_{p, q}\left(c_{2}\right)+\Phi_{p, q}\left(c_{1}, c_{3}\right)=0, \\
\cdots \\
\sum_{t=1}^{l-1} a_{t}^{2} b_{2 l-2 t}+2 \sum_{t=1}^{l-1} a_{t} \sum_{\delta=t+1}^{2 l-(t+1)} a_{\delta} b_{2 l-\delta-t}+Q_{p, q}\left(c_{l}\right)+\sum_{t=1}^{l-1} \Phi_{p, q}\left(c_{t}, c_{2 l-t}\right)=0, \\
\epsilon b_{1}^{2 l+1}+\sum_{t=1}^{l} a_{t}^{2} b_{2 l+1-2 t}+2 \sum_{t=1}^{l-1} a_{s} \sum_{\delta=t+1}^{2 l+1-(t+1)} a_{\delta} b_{2 l+1-\delta-t}+\sum_{t=1}^{l} \Phi_{p, q}\left(c_{t}, c_{2 l+1-t}\right)=0 .
\end{array}\right.
$$

Proceeding as in the proof of proposition 7.1 (see also the proof of proposition 6.10), we obtain

$$
\begin{aligned}
\beta^{G}\left(A_{k}^{0}\left(h_{k+1}\right)\right)= & u^{3 l+2+(l+1)(p+q)} \frac{u^{l(p+q-1)}-1}{u^{p+q-1}-1}\left(\beta^{G}\left(Y_{p, q} \backslash\{0\}\right)\right) \\
& +\sum_{t=0}^{l-2} u^{t(3+p+q)} u^{(k-1-2 t)(p+q+1)+3}+u^{(l-1)(3+p+q)} \beta^{G}\left(S_{3}^{0}\right),
\end{aligned}
$$

if $S_{3}^{0}$ denotes the algebraic set defined by the equation $\epsilon b_{1}^{2 l+1}+a_{1}^{2} b_{1}=0$, the variables $a_{2}, a_{3}$, $b_{2}, b_{3}$ as well as the vectors $c_{2}, c_{3}$ being free. Hence the desired result.

If we suppose $k$ even, $k=2 l$, the set $A_{k}^{0}\left(h_{k+1}\right)$ is described by the system

$$
\left\{\begin{array}{l}
Q_{p, q}\left(c_{1}\right)=0, \\
a_{1}^{2} b_{1}+\Phi_{p, q}\left(c_{1}, c_{2}\right)=0, \\
\cdots \\
\sum_{t=1}^{l-1} a_{t}^{2} b_{2 l-1-2 t}+2 \sum_{t=1}^{l-2} a_{s} \sum_{\delta=t+1}^{2 l-1+(t+1)} a_{\delta} b_{2 l-1-\delta-t}+\sum_{t=1}^{l-1} \Phi_{p, q}\left(c_{t}, c_{2 l-1-t}\right)=0, \\
\epsilon b_{1}^{2 l}+\sum_{t=1}^{l-1} a_{t}^{2} b_{2 l-2 t}+2 \sum_{t=1}^{l-1} a_{t} \sum_{\delta=t+1}^{2 l-(t+1)} a_{\delta} b_{2 l-\delta-t}+Q_{p, q}\left(c_{l}\right)+\sum_{t=1}^{l-1} \Phi_{p, q}\left(c_{t}, c_{2 l-t}\right)=0,
\end{array}\right.
$$


and we have

$$
\beta^{G}\left(A_{k}^{0}\left(h_{k+1}\right)\right)=u^{3 l+(l+1)(p+q)} \frac{u^{(l-1)(p+q-1)}-1}{u^{p+q-1}-1}\left(\beta^{G}\left(Y_{p, q} \backslash\{0\}\right)+1\right)+u^{(l-1)(3+p+q)} \beta^{G}\left(S_{2}^{0}\right),
$$

where $S_{2}^{0}$ is the algebraic set given by the equation $\epsilon b_{1}^{2 l}+Q_{p, q}\left(c_{1}\right)=0$, with free variables $a_{1}, a_{2}, b_{2}$ and free vector $c_{2}$.

Since, in our present framework, the quantity $\beta^{G}\left(Y_{p, q}\right)$ is the same for $h_{k+1}$ and $g_{k}$, we are reduced to study the equivariant virtual Poincaré series of the $G$-algebraic sets $\left\{h_{k+1}\left(x_{2}, x_{3}, 0\right)=\right.$ $0\}$ and $\left\{r_{k}\left(x_{1}, x_{2}, 0\right)=0\right\}$ if $k$ is odd, resp. $\left\{h_{k+1}\left(0, x_{3}, y\right)=0\right\}$ and $\left\{r_{k}\left(0, x_{2}, y\right)=0\right\}$ if $k$ is even.

Notice that, if $k$ is even, $\beta^{G}\left(\left\{h_{k+1}\left(0, x_{3}, y\right)=0\right\}\right)$ and $\beta^{G}\left(\left\{r_{k}\left(0, x_{2}, y\right)=0\right\}\right)$ have been already computed in lemma 6.11 : if $k$ is even and if $p>q+1$ and $\eta=+1$ or $q>p+1$ and $\eta=$ -1 , the equivariant virtual Poincaré series $\beta^{G}\left(\left\{h_{k+1}\left(0, x_{3}, y\right)=0\right\}\right)$ and $\beta^{G}\left(\left\{r_{k}\left(0, x_{2}, y\right)=0\right\}\right)$ are equal and therefore $\beta^{G}\left(A_{k}\left(h_{k+1}\right)\right)=\beta^{G}\left(A_{k}\left(r_{k}\right)\right)$.

Now, in the next lemma, we compute $\left\{h_{k+1}\left(x_{2}, x_{3}, 0\right)=0\right\}$ and $\left\{r_{k}\left(x_{1}, x_{2}, 0\right)=0\right\}$ if $k$ is odd, $k=2 l+1$ :

Lemma 7.6. We have

$$
\beta^{G}\left(\left\{h_{2 l+2}\left(x_{2}, x_{3}, 0\right)=0\right\}\right)=\beta^{G}\left(\left\{x_{2}^{2}+\epsilon x_{3}^{2}=0\right\}\right)-\frac{u}{u-1}+\frac{u^{2}}{u-1},
$$

where the latter set is considered as an algebraic subset of $\mathbb{R}^{2}$ on which the group $G$ acts trivially, and

$$
\beta^{G}\left(\left\{r_{2 l+1}\left(x_{1}, x_{2}, 0\right)=0\right\}\right)=\beta^{G}\left(\left\{x_{1}^{2}+\epsilon x_{2}^{2}=0\right\}\right)-\frac{u}{u-1}+\frac{u^{2}}{u-1},
$$

where the latter set is considered as an algebraic subset of $\mathbb{R}^{2}$ on which the group $G$ acts only changing the sign of the coordinate $x_{1}$.

Proof. We make the computation for $\beta^{G}\left(\left\{r_{2 l+1}\left(x_{1}, x_{2}, 0\right)=0\right\}\right)$.

Consider the equation $x_{1}^{2} x_{2}+\epsilon x_{2}^{2 l+1}=0$. If $x_{2} \neq 0$, it is equivalent to $x_{1}^{2}+\epsilon x_{2}^{2 l}=0$, and if $x_{2}=0$, it becomes trivial. Consequently,

$$
\beta^{G}\left(\left\{r_{2 l+1}\left(x_{1}, x_{2}, 0\right)=0\right\}\right)=\beta^{G}\left(\left\{x_{1}^{2}+\epsilon x_{2}^{2 l}=0\right\} \backslash\{(0,0)\}\right)+\frac{u^{2}}{u-1},
$$

and we use lemma 6.11 to write $\beta^{G}\left(\left\{x_{1}^{2}+\epsilon x_{2}^{2 l}=0\right\}\right)=\beta^{G}\left(\left\{x_{1}^{2}+\epsilon x_{2}^{2}=0\right\}\right)-(l-1) \beta^{G}\left(\left\{x_{1}^{2}=\right.\right.$ $0\})+(l-1) \beta^{G}(\{(0,0)\})=\beta^{G}\left(\left\{x_{1}^{2}+\epsilon x_{2}^{2}=0\right\}\right)$ (recall also that the equivariant virtual Poincaré series of a point is $\left.\frac{u}{u-1}\right)$.

If $\epsilon=+1$, the sets $\left.\left\{x_{2}^{2}+x_{3}^{2}=0\right\}\right)$ and $\left\{x_{1}^{2}+x_{2}^{2}=0\right\}$ are both reduced to a single point. On the other hand, if $\epsilon=-1$, we have $\beta^{G}\left(\left\{x_{2}^{2}-x_{3}^{2}=0\right\}\right)=\frac{2 u^{2}-u}{u-1}$ whereas $\beta^{G}\left(\left\{x_{1}^{2}-x_{2}^{2}=0\right\}\right)=$ $\frac{u^{2}-u+1}{u-1}$ (see proposition 5.1). As a consequence :

Corollary 7.7. If $k$ is odd and if $\epsilon=-1$, the germs $h_{k+1}$ and $r_{k}$ are not G-blow-Nash equivalent. 
If $p>q+1$ and $\eta=+1$ or $q>p+1$ and $\eta=-1$, and if $k$ is even or $k$ is odd and $\epsilon=+1$, the coefficients $\beta^{G}\left(A_{k}\left(h_{k+1}\right)\right)$ and $\beta^{G}\left(A_{k}\left(r_{k}\right)\right)$ of the respective naive equivariant zeta functions of $h_{k+1}$ and $r_{k}$ are equal. We are then led to look at the coefficients $\beta^{G}\left(A_{k}^{\xi}\left(h_{k+1}\right)\right)$ and $\beta^{G}\left(A_{k}^{\xi}\left(r_{k}\right)\right)$ of their respective equivariant zeta functions with signs.

\subsection{Computation of $\beta^{G}\left(A_{k}^{\xi}\left(h_{k+1}\right)\right)$ and $\beta^{G}\left(A_{k}^{\xi}\left(r_{k}\right)\right)$}

For the cases listed above, we consider the quantities $\beta^{G}\left(A_{k}^{\xi}\left(h_{k+1}\right)\right)$ and $\beta^{G}\left(A_{k}^{\xi}\left(r_{k}\right)\right)$, expressed by the following formulae (just follow the steps of computation of the proof of proposition 7.5) :

Proposition 7.8. Suppose $k \geq 3$. Then

$$
\beta^{G}\left(A_{k}^{\xi}\left(h_{k+1}\right)\right)=\left\{\begin{array}{l}
u^{3 l+2+(l+1)(p+q)} \frac{u^{l(p+q-1)}-1}{u^{p+q-1}-1}\left(\beta^{G}\left(Y_{p, q} \backslash\{0\}\right)\right) \\
+u^{3 l+1+(l+2)(p+q)} \frac{u^{(l-1)(p+q-1}-1}{u^{p+q-1}-1}+u^{3 l+1+(l+1)(p+q)} \beta^{G}\left(\left\{h_{k+1}\left(x_{2}, x_{3}, 0\right)=\xi\right\}\right) \text { if } k=2 l+1, \\
\quad+u^{3 l+l(p+q)} \beta^{G}\left(\left\{h_{k+1}\left(0, x_{3}, y\right)=\xi\right\}\right) \text { if } k=2 l,
\end{array}\right.
$$

and

$$
\beta^{G}\left(A_{k}^{\xi}\left(r_{k}\right)\right)=\left\{\begin{array}{c}
u^{3 l+2+(l+1)(p+q)} \frac{u^{l(p+q-1)}-1}{u^{p+q-1}-1}\left(\beta^{G}\left(Y_{p, q} \backslash\{0\}\right)\right) \\
\quad+u^{3 l+1+(l+2)(p+q)} \frac{u^{(l-1)(p+q-1)}-1}{u^{p+q-1}-1}+u^{3 l+1+(l+1)(p+q)} \beta^{G}\left(\left\{r_{k}\left(x_{1}, x_{2}, 0\right)=\xi\right\}\right) \text { if } k=2 l+1, \\
u^{3 l+(l+1)(p+q)} \frac{u^{(l-1)(p+q-1)}-1}{u^{p+q-1}-1}\left(\beta^{G}\left(Y_{p, q} \backslash\{0\}\right)+1\right)+u^{3 l+l(p+q)} \beta^{G}\left(\left\{r_{k}\left(0, x_{2}, y\right)=\xi\right\}\right) \text { if } k=2 l .
\end{array}\right.
$$

If $k$ is even, we can use the formulae of lemma 6.15 and the same arguments as in the proofs of propositions 6.17 and 6.18 in order to establish the following facts :

Proposition 7.9. Suppose $k$ is even, $k=2 l \geq 4$.

1. If $p>q+1$ and $\eta=\epsilon=+1$ or $q>p+1$ and $\eta=\epsilon=-1$, then $\beta^{G}\left(A_{k}^{\xi}\left(h_{k+1}\right)\right)=$ $\beta^{G}\left(A_{k}^{\xi}\left(r_{k}\right)\right)$.

2. - If $p>q+1, \eta=+1$ and $\epsilon=-1$, the equality $\beta^{G}\left(A_{k}^{\xi}\left(h_{k+1}\right)\right)=\beta^{G}\left(A_{k}^{\xi}\left(r_{k}\right)\right)$ is true if and only if the equivariant virtual Poincaré series of the algebraic subsets $\left\{-x_{3}^{2 l}+y^{2}+\sum_{i=1}^{K-1} y_{i}^{2}=\xi\right\} \subset \mathbb{R}^{K+1}, K:=p-q$, equipped with the action of $G$ changing only the sign of $y$, and $\left\{-x_{2}^{2 l}+\sum_{i=1}^{K} z_{i}^{2}=\xi\right\} \subset \mathbb{R}^{K+1}$, equipped with the trivial action of $G$, are equal.

- If $q>p+1, \eta=-1$ and $\epsilon=+1$, we have $\beta^{G}\left(A_{k}^{\xi}\left(h_{k+1}\right)\right)=\beta^{G}\left(A_{k}^{\xi}\left(r_{k}\right)\right)$ if and only if $\beta^{G}\left(\left\{x_{3}^{2 l}-y^{2}-\sum_{i=1}^{K-1} y_{i}^{2}=\xi\right\}\right)=\beta^{G}\left(\left\{x_{2}^{2 l}-\sum_{i=1}^{K} z_{i}^{2}=\xi\right\}\right)$.

If $k$ is odd, $k=2 l+1$ and $\epsilon=+1$, and if $p>q+1$ and $\eta=+1$ or $q>p+1$ and $\eta=-1$, we are reduced to compare $\beta^{G}\left(\left\{h_{k+1}\left(x_{2}, x_{3}, 0\right)=\xi\right\}\right)=\beta^{G}\left(\left\{x_{2}^{2} x_{3}+x_{3}^{2 l+1}=\xi\right\}\right)$ and $\beta^{G}\left(\left\{r_{k}\left(x_{1}, x_{2}, 0\right)=\xi\right\}\right)=\beta^{G}\left(\left\{x_{1}^{2} x_{2}+x_{2}^{2 l+1}=\xi\right\}\right)$. We are going to show that these two quantities are equal and therefore : 
Proposition 7.10. If $p>q+1$ and $\eta=+1$ or $q>p+1$ and $\eta=-1$, and if $k$ is odd and $\epsilon=+1$, then $\beta^{G}\left(A_{k}^{\xi}\left(h_{k+1}\right)\right)=\beta^{G}\left(A_{k}^{\xi}\left(r_{k}\right)\right)$.

Proof. We compute the equivariant virtual Poincaré series of the nonsingular curve $C:=$ $\left\{x_{0}^{2} y_{0}+y_{0}^{2 l+1}=\xi\right\}$ of $\mathbb{R}^{2}$, on which the group $G$ acts only changing the sign of the first coordinate $x_{0}$, resp. trivially.

First suppose the action of $G$ is the former one. Suppose also $l \geq 2$. We equivariantly compactify $C$ in the projective space $\mathbb{P}^{2}(\mathbb{R})$ with homogeneous coordinates $[X: Y: Z]$, on which $G$ acts via the involution $[X: Y: Z] \mapsto[-X: Y: Z]=[X:-Y:-Z]$. We denote $\Gamma:=\left\{X^{2} Y Z^{2 l-2}+Y^{2 l+1}=\xi Z^{2 l+1}\right\}$ this compactification and $p:=[1: 0: 0]$ the point at infinity.

The equivariant compactification $\Gamma$ is singular at the fixed point $p$ as one can see in the globally invariant chart $X \neq 0$. If $\left(y_{0}, z_{0}\right)$ are the coordinates in this chart, the group $G$ acting via the involution $\left(y_{0}, z_{0}\right) \mapsto\left(-y_{0},-z_{0}\right)$, we denote by $C^{\prime}$ the curve $\Gamma \cap\{X \neq 0\}=$ $\left\{y_{0} z_{0}^{2 l-2}+y_{0}^{2 l+1}=\xi z_{0}^{2 l+1}\right\}$ (the point at infinity is the fixed point $q=[0: \xi: 1]$ of $C$ ).

Equivariantly blowing-up $\Gamma$ at $p$ resolves the singularity : in the chart $y_{0}=u_{0} v_{0}, z_{0}=v_{0}$, where the action of $G$ is given by $\left(u_{0}, v_{0}\right) \mapsto\left(u_{0},-v_{0}\right)$, the equation of the strict transform is $u_{0}+u_{0}^{2 l+1} v_{0}^{2}-\xi v_{0}^{2}=0$ and it intersects the exceptional divisor at the single point $p_{0}$ with coordinates $\left(u_{0}, v_{0}\right)=(0,0)$. The resolved compact $G$-variety, denoted by $\widetilde{\Gamma}$, is equivariantly homeomorphic to a circle equipped with an action of $G$ fixing the two points $p_{0}$ and $q$.

As a conclusion, we have

$$
\beta^{G}(C)=\beta(\Gamma \backslash\{p\})=\beta^{G}\left(\widetilde{\Gamma} \backslash\left\{p_{0}\right\}\right)=\beta^{G}(\widetilde{\Gamma})-\beta^{G}\left(\left\{p_{0}\right\}\right)=u+2 \frac{u}{u-1}-\frac{u}{u-1}=\frac{u^{2}}{u-1}
$$

(see remark 4.1).

If $l=1$, the point $p$ of $\Gamma$ is not singular and $\Gamma$ is a compact nonsingular $G$-variety equivariantly homeomorphic to a circle with two fixed points $p$ and $q$.

If now we suppose that the affine space $\mathbb{R}^{2}$ with coordinates $\left(x_{0}, y_{0}\right)$ is equipped with the trivial action of $G$, we will obtain the same expression for $\beta^{G}(C)$ since the equivariant homology of a circle is the same as soon as there is at least one fixed point.

Consequently, the equivariant virtual Poincaré series $\beta^{G}\left(\left\{h_{k+1}\left(x_{2}, x_{3}, 0\right)=\xi\right\}\right)=\beta^{G}\left(\left\{x_{2}^{2} x_{3}+\right.\right.$ $\left.\left.x_{3}^{2 l+1}=\xi\right\}\right)$ and $\beta^{G}\left(\left\{r_{k}\left(x_{1}, x_{2}, 0\right)=\xi\right\}\right)=\beta^{G}\left(\left\{x_{1}^{2} x_{2}+x_{2}^{2 l+1}=\xi\right\}\right)$ are equal and then $\beta^{G}\left(A_{k}^{\xi}\left(h_{k+1}\right)\right)=\beta^{G}\left(A_{k}^{\xi}\left(r_{k}\right)\right)$.

In the next paragraph, we will look at the last part of the respective equivariant zeta functions of $h_{k+1}$ and $r_{k}$. Still supposing that $p>q+1$ and $\eta=+1$ or $q>p+1$ and $\eta=-1$, we will show that, if $k$ is even or $k$ is odd and $\epsilon=+1$, their comparison reduces as in proposition 7.9 . 


\subsection{The last terms of the equivariant zeta functions}

Suppose $p>q+1$ and $\eta=+1$ or $q>p+1$ and $\eta=-1$. Suppose that $k$ is even or that $k$ is odd and $\epsilon=+1$. The naive equivariant zeta functions of $h_{k+1}$ and $r_{k}$ are equal :

Proposition 7.11. For all $M>k$, we have $\beta^{G}\left(A_{M}\left(h_{k+1}\right)\right)=\beta^{G}\left(A_{M}\left(r_{k}\right)\right)$.

Proof. Let $M$ be greater than $k$. We prove that $\beta^{G}\left(A_{M}^{0}\left(h_{k+1}\right)\right)=\beta^{G}\left(A_{M}^{0}\left(r_{k}\right)\right)$.

Suppose $k$ to be even, $k=2 l$. Consider the system of equations describing $A_{M}^{0}\left(h_{k+1}\right)$ and $A_{M}^{0}\left(r_{k}\right)$. The same computations as in the proofs of propositions 7.1 and $7.5 \mathrm{bring}$, in both expressions of $\beta^{G}\left(A_{M}^{0}\left(h_{k+1}\right)\right)$ and $\beta^{G}\left(A_{M}^{0}\left(r_{k}\right)\right)$, an equal contribution of $\beta^{G}\left(Y_{p, q} \backslash\{0\}\right)$ and a contribution of the equivariant virtual Poincaré series of a set defined by a system whose first equation is $\epsilon b_{1}^{k}+Q_{p, q}\left(c_{1}\right)=0$. Stratifying this last algebraic set with the subsets $\left\{c_{1}^{1}=\ldots=c_{1}^{i-1}=0, c_{1}^{i} \neq 0\right\}, i=1, \ldots, p+q$, and $\left\{c_{1}=0\right\}$ provides a contribution of $\beta^{G}\left(\left\{h_{k+1}\left(0, x_{3}, y\right)=0\right\} \backslash\{0\}\right)$, resp. $\beta^{G}\left(\left\{r_{k}\left(0, x_{2}, y\right)=0\right\} \backslash\{0\}\right)$ (it is the same quantity in our hypothesis) and we are led to the further condition $c_{1}=0$, and then $b_{1}=0$, in the previous system.

Now, stratify with the subsets $\left\{a_{1} \neq 0\right\}$ (this will provide an equal contribution for $h_{k+1}$ and $r_{k}$ ) and $\left\{a_{1}=0\right\}$. If $a_{1}=0$, shifting by -1 the indices of the remaining variables $a_{i}$ and $c_{i}$, we obtain a new system whose first equations are, if $M \geq 2 k$ :

$$
\left\{\begin{array}{l}
Q_{p, q}\left(c_{1}\right)=0, \\
\Phi_{p, q}\left(c_{1}, c_{2}\right)=0, \\
a_{1}^{2} b_{2}+Q_{p, q}\left(c_{2}\right)+\Phi_{p, q}\left(c_{1}, c_{3}\right)=0, \\
a_{1}^{2} b_{3}+2 a_{1} a_{2} b_{2}+\Phi_{p, q}\left(c_{1}, c_{4}\right)+\Phi_{p, q}\left(c_{2}, c_{3}\right)=0, \\
\cdots \\
\sum_{t=1}^{l-2} a_{t}^{2} b_{2 l-1-2 t}+2 \sum_{t=1}^{l-2} a_{s} \sum_{\delta=t+1}^{2 l-2-(t+1)} a_{\delta} b_{2 l-1-\delta-t}+\sum_{t=1}^{l-1} \Phi_{p, q}\left(c_{t}, c_{2 l-1-t}\right)=0, \\
\epsilon b_{2}^{2 l}+\sum_{t=1}^{l-1} a_{t}^{2} b_{2 l-2 t}+2 \sum_{t=1}^{l-2} a_{t} \sum_{\delta=t+1}^{2 l-1-(t+1)} a_{\delta} b_{2 l-\delta-t}+Q_{p, q}\left(c_{l}\right)+\sum_{t=1}^{l-1} \Phi_{p, q}\left(c_{t}, c_{2 l-t}\right)=0 .
\end{array}\right.
$$

These equations can be obtained from the system defining $A_{k}^{0}\left(h_{k+1}\right)$, by replacing the term $\epsilon b_{1}^{2 l}$ with $\epsilon b_{2}^{2 l}$ in the last equation and imposing $b_{1}$ to be 0 in the other ones.

Therefore, a similar process as above can be applied and provides further equal contributions for $\beta^{G}\left(A_{M}^{0}\left(h_{k+1}\right)\right)$ and $\beta^{G}\left(A_{M}^{0}\left(r_{k}\right)\right)$. In any case, the final equation will be either $Q_{p, q}\left(c_{1}\right)=0$, $\epsilon b_{j}^{2 l}+Q_{p, q}\left(c_{1}\right)=0$ or trivial, so that the induced respective contributions are equal as well.

As a consequence, $\beta^{G}\left(A_{M}^{0}\left(h_{k+1}\right)\right)=\beta^{G}\left(A_{M}^{0}\left(r_{k}\right)\right)$ and $\beta^{G}\left(A_{M}\left(h_{k+1}\right)\right)=\beta^{G}\left(A_{M}\left(r_{k}\right)\right)$.

If $k$ is odd, $k=2 l+1$, and $\epsilon=+1$, from the initial system of equations defining $A_{M}^{0}\left(h_{k+1}\right)$ and $A_{M}^{0}\left(r_{k}\right)$, we are reduced to consider a system whose first equation is $b_{1}^{2 l+1}+a_{1}^{2} b_{1}=0$ (see the proof of proposition 7.5). Therefore $b_{1}=0$. Stratifying with the subsets $\left\{a_{1} \neq 0\right\}$ and $\left\{a_{1}=0\right\}$, we then get, after a renaming of the variables, a system whose first non trivial 
equations are, if $M \geq 2 k$ :

$$
\left\{\begin{array}{l}
Q_{p, q}\left(c_{1}\right)=0 \\
\Phi_{p, q}\left(c_{1}, c_{2}\right)=0, \\
a_{1}^{2} b_{2}+Q_{p, q}\left(c_{2}\right)+\Phi_{p, q}\left(c_{1}, c_{3}\right)=0, \\
a_{1}^{2} b_{3}+2 a_{1} a_{2} b_{2}+\Phi_{p, q}\left(c_{1}, c_{4}\right)+\Phi_{p, q}\left(c_{2}, c_{3}\right)=0, \\
\cdots \\
\sum_{t=1}^{l-1} a_{t}^{2} b_{2 l+1-2 t}+2 \sum_{t=1}^{l-1} a_{s} \sum_{\delta=t+1}^{2 l-(t+1)} a_{\delta} b_{2 l+1-\delta-t}+\sum_{t=1}^{l} \Phi_{p, q}\left(c_{t}, c_{2 l+1-t}\right)=0, \\
b_{2}^{2 l+1}+\sum_{t=1}^{l} a_{t}^{2} b_{2 l+2-2 t}+2 \sum_{t=1}^{l-1} a_{t} \sum_{\delta=t+1}^{2 l+1-(t+1)} a_{\delta} b_{2 l+2-\delta-t}+Q_{p, q}\left(c_{l+1}\right)+\sum_{t=1}^{l} \Phi_{p, q}\left(c_{t}, c_{2 l+2-t}\right)=0 .
\end{array}\right.
$$

Repeating the process provides further equal contributions for $\beta^{G}\left(A_{M}^{0}\left(h_{k+1}\right)\right)$ and $\beta^{G}\left(A_{M}^{0}\left(r_{k}\right)\right)$ and, if $M \geq 2 k$, we are led to a new system whose first equation is $b_{2}^{2 l+1}+Q_{p, q}\left(c_{1}\right)=0$. We will show in lemma 7.12 below that the respective induced contributions are equal.

In any case, these repeated steps of computations will eventually allow us to consider a single equation, which will be either $Q_{p, q}\left(c_{1}\right)=0, b_{j}^{2 l+1}+a_{1}^{2} b_{j}=0, b_{j}^{2 l+1}+Q_{p, q}\left(c_{1}\right)=0$ or trivial.

Consequently, if $k$ is odd, $\beta^{G}\left(A_{M}^{0}\left(h_{k+1}\right)\right)=\beta^{G}\left(A_{M}^{0}\left(r_{k}\right)\right)$ and $\beta^{G}\left(A_{M}\left(h_{k+1}\right)\right)=\beta^{G}\left(A_{M}\left(r_{k}\right)\right)$ as well.

Lemma 7.12. Suppose that $k$ is odd, $k=2 l+1, p>q+1$ and $\eta=+1$ (the property will also be true if $q>p+1$ and $\eta=-1$ ). Then

$$
\beta^{G}\left(\left\{h_{k+1}\left(0, x_{3}, y\right)=0\right\}\right)=\beta^{G}\left(\left\{r_{k}\left(0, x_{2}, y\right)=0\right\}\right) .
$$

Proof. Applying successive blowings-up as in the proof of lemma 6.11, we obtain

$$
\begin{aligned}
\beta^{G}\left(\left\{h_{k+1}\left(0, x_{3}, y\right)=0\right\}\right) & =\beta^{G}\left(\left\{\epsilon x_{3}+Q_{p, q}(y)=0\right\}\right)-k \beta^{G}\left(\left\{Q_{p, q}(y)=0\right\}\right)+k \beta^{G}(\{0\}) \\
& =\beta^{G}\left(\mathbb{R}^{p+q}\right)-k \beta^{G}\left(\left\{Q_{p, q}(y)=0\right\}\right)+k \beta^{G}(\{0\}) .
\end{aligned}
$$

We have the same expression for $\beta^{G}\left(\left\{r_{k}\left(0, x_{2}, y\right)=0\right\}\right)$ and therefore, since $p>q+1$ and $\eta=+1$, the two quantities are equal.

As for the last part of the equivariant zeta functions with signs of $h_{k+1}$ and $r_{k}$, adapting the computations of the proof of proposition 7.11, we obtain the following (still under our current hypothesis) :

Proposition 7.13. 1. Suppose $k$ is even.

- If $\eta=\epsilon$, then, for all $M>k, \beta^{G}\left(A_{M}^{\xi}\left(h_{k+1}\right)\right)=\beta^{G}\left(A_{M}^{\xi}\left(r_{k}\right)\right)$ and consequently the respective equivariant zeta functions with signs of $h_{k+1}$ and $r_{k}$ are equal.

- If $\eta=+1, \epsilon=-1$, we have the equality $Z_{h_{k+1}}^{G, \xi}(u, T)=Z_{r_{k}}^{G, \xi}(u, T)$ if and only if $\beta^{G}\left(\left\{-x_{3}^{k}+y^{2}+\sum_{i=1}^{K-1} y_{i}^{2}=\xi\right\}\right)=\beta^{G}\left(\left\{-x_{2}^{k}+\sum_{i=1}^{K} z_{i}^{2}=\xi\right\}\right)$ (the former set is a subset of $\mathbb{R}^{K+1}$ equipped with the action of $G$ only changing the sign of $y$ and the latter set is a subset of $\mathbb{R}^{K+1}$ equipped with the trivial action of $G$ ). 
- If $\eta=-1, \epsilon=+1$, we have the equality $Z_{h_{k+1}}^{G, \xi}(u, T)=Z_{r_{k}}^{G, \xi}(u, T)$ if and only if $\beta^{G}\left(\left\{x_{3}^{k}-y^{2}-\sum_{i=1}^{K-1} y_{i}^{2}=\xi\right\}\right)=\beta^{G}\left(\left\{x_{2}^{k}-\sum_{i=1}^{K} z_{i}^{2}=\xi\right\}\right)$.

2. Suppose $k$ is odd and $\epsilon=+1$.

- If $\eta=+1$, the equality $Z_{h_{k+1}}^{G, \xi}(u, T)=Z_{r_{k}}^{G, \xi}(u, T)$ is true if and only if the quantities $\beta^{G}\left(\left\{x_{3}^{k}+y^{2}+\sum_{i=1}^{K-1} y_{i}^{2}=\xi\right\}\right)$ and $\beta^{G}\left(\left\{x_{2}^{k}+\sum_{i=1}^{K} z_{i}^{2}=\xi\right\}\right)$ are equal.

- If $\eta=-1$, the equality $Z_{h_{k+1}}^{G, \xi}(u, T)=Z_{r_{k}}^{G, \xi}(u, T)$ is true if and only if the quantities $\beta^{G}\left(\left\{x_{3}^{k}-y^{2}-\sum_{i=1}^{K-1} y_{i}^{2}=\xi\right\}\right)$ and $\beta^{G}\left(\left\{x_{2}^{k}-\sum_{i=1}^{K} z_{i}^{2}=\xi\right\}\right)$ are equal.

Proof. Let $M$ be greater than $k$. Since the system describing $A_{M}^{\xi}\left(h_{k+1}\right)$ and $A_{M}^{\xi}\left(r_{k}\right)$ is obtained from the one defining $A_{M}^{0}\left(h_{k+1}\right)$ and $A_{M}^{0}\left(r_{k}\right)$ by replacing 0 by $\xi$ in the right member of the last equation, we are reduced, as in the proof of proposition 7.11, to consider a single equation.

If $k$ is even, $k=2 l$, this equation is either $Q_{p, q}\left(c_{1}\right)=\xi, \epsilon b_{j}^{2 l}+Q_{p, q}\left(c_{1}\right)=\xi$ or an equation with no solution. Under our current hypothesis, the quantity $\beta^{G}\left(Y_{p, q}^{\xi}\right)$ is the same for $h_{k+1}$ and $r_{k}$. If $\epsilon=\eta$, we can show, as in the proof of proposition 6.17, using the formulae of lemma 6.15 , that $\beta^{G}\left(\left\{h_{k+1}\left(0, x_{3}, y\right)=\xi\right\}\right)=\beta^{G}\left(\left\{r_{k}\left(0, x_{2}, y\right)=\xi\right\}\right)$. If $\epsilon=-\eta$, we also use lemma 6.15 to obtain the desired equivalences.

If $k$ is odd and $\epsilon=+1$, the final equation is either $Q_{p, q}\left(c_{1}\right)=\xi, b_{j}^{2 l+1}+a_{1}^{2} b_{j}=\xi, b_{j}^{2 l+1}+$ $Q_{p, q}\left(c_{1}\right)=\xi$ or an equation with no solution. The quantity $\beta^{G}\left(Y_{p, q}^{\xi}\right)$ is the same for $h_{k+1}$ and $r_{k}$ and we showed in proposition 7.10 that $\beta^{G}\left(\left\{h_{k+1}\left(x_{2}, x_{3}, 0\right)=\xi\right\}\right)=\beta^{G}\left(\left\{r_{k}\left(x_{1}, x_{2}, 0\right)=\xi\right\}\right)$. Finally, we can obtain formulae similar to the ones in lemma 6.15 for $\beta^{G}\left(\left\{h_{k+1}\left(0, x_{3}, y\right)=\xi\right\}\right)$ and $\beta^{G}\left(\left\{r_{k}\left(0, x_{2}, y\right)=\xi\right\}\right)$ if $k$ is odd and this provides the desired equivalences.

\subsection{Conclusion}

We gather the obtained results in the following statement:

Theorem 7.14. Let $k \geq 3$. Suppose that the invariant germs

$$
h_{k+1}=x_{2}^{2} x_{3}+\epsilon x_{3}^{k}+\eta x_{1}^{2}+Q \text { and } r_{k}=x_{1}^{2} x_{2}+\epsilon x_{2}^{k}+\eta^{\prime} x_{3}^{2}+Q^{\prime}
$$

have, up to permutation of all variables, the same quadratic part, with $p$ signs + and $q$ signs - .

1. If

- $p \leq q, \eta=+1$ or $q \leq p, \eta=-1$,

- $p=q+1$ or $q=p+1$,

- $k$ is odd, $\epsilon=-1$,

then $h_{k+1}$ and $r_{k}$ are not $G$-blow-Nash equivalent.

2. If $k$ is even and if $p>q+1, \eta=+1, \epsilon=+1$ or $q>p+1, \eta=-1, \epsilon=-1$, then $Z_{h_{k+1}}^{G}(u, T)=Z_{r_{k}}^{G}(u, T)$ and $Z_{h_{k+1}}^{G, \xi}(u, T)=Z_{r_{k}}^{G, \xi}(u, T)$. 
3. - If $k$ is even and if $p>q+1, \eta=+1, \epsilon=-1$, then $Z_{h_{k+1}}^{G}(u, T)=Z_{r_{k}}^{G}(u, T)$. Furthermore, $Z_{h_{k+1}}^{G, \xi}(u, T)=Z_{r_{k}}^{G, \xi}(u, T)$ if and only if $\beta^{G}\left(\left\{-x_{3}^{k}+y^{2}+\sum_{i=1}^{K-1} y_{i}^{2}=\xi\right\}\right)=$ $\beta^{G}\left(\left\{-x_{2}^{k}+\sum_{i=1}^{K} z_{i}^{2}=\xi\right\}\right)$.

- If $k$ is even and if $q>p+1, \eta=-1, \epsilon=+1$, then $Z_{h_{k+1}}^{G}(u, T)=Z_{r_{k}}^{G}(u, T)$. Furthermore, $Z_{h_{k+1}}^{G, \xi}(u, T)=Z_{r_{k}}^{G, \xi}(u, T)$ if and only if $\beta^{G}\left(\left\{x_{3}^{k}-y^{2}-\sum_{i=1}^{K-1} y_{i}^{2}=\xi\right\}\right)=$ $\beta^{G}\left(\left\{x_{2}^{k}-\sum_{i=1}^{K} z_{i}^{2}=\xi\right\}\right)$.

- If $k$ is odd and if $p>q+1, \eta=+1, \epsilon=+1$, then $Z_{h_{k+1}}^{G}(u, T)=Z_{r_{k}}^{G}(u, T)$. Furthermore, $Z_{h_{k+1}}^{G, \xi}(u, T)=Z_{r_{k}}^{G, \xi}(u, T)$ if and only if $\beta^{G}\left(\left\{x_{3}^{k}+y^{2}+\sum_{i=1}^{K-1} y_{i}^{2}=\xi\right\}\right)=$ $\beta^{G}\left(\left\{x_{2}^{k}+\sum_{i=1}^{K} z_{i}^{2}=\xi\right\}\right)$.

- If $k$ is odd and if $q>p+1, \eta=-1, \epsilon=+1$, then $Z_{h_{k+1}}^{G}(u, T)=Z_{r_{k}}^{G}(u, T)$. Furthermore, $Z_{h_{k+1}}^{G, \xi}(u, T)=Z_{r_{k}}^{G, \xi}(u, T)$ if and only if $\beta^{G}\left(\left\{x_{3}^{k}-y^{2}-\sum_{i=1}^{K-1} y_{i}^{2}=\xi\right\}\right)=$ $\beta^{G}\left(\left\{x_{2}^{k}-\sum_{i=1}^{K} z_{i}^{2}=\xi\right\}\right)$.

Remark 7.15. If we forget the $G$-actions, the virtual Poincaré polynomials of the algebraic subsets $\left\{x^{2 l+1}+\sum_{i=1}^{K} y_{i}^{2}=\xi\right\}$ and $\left\{x^{2 l+1}-\sum_{i=1}^{K} y_{i}^{2}=\xi\right\}$ of $\mathbb{R}^{K+1}, \xi= \pm 1$, can also be computed using the invariance of the virtual Poincaré polynomial under bijection with $\mathcal{A S}$ graph (see remark 6.19).

\section{The germs $E_{6}$ and $F_{4}$}

Finally, we study the classification with respect to $G$-blow-Nash equivalence of the families

$$
\varphi^{\epsilon}(x):= \pm x_{1}^{2}+x_{2}^{3}+\epsilon x_{3}^{4}+Q
$$

and

$$
\omega^{\epsilon}(x):=\epsilon x_{1}^{4}+x_{2}^{3}+ \pm x_{3}^{3}+Q^{\prime}
$$

where $\epsilon \in\{-1 ;+1\}$.

If two germs $\varphi^{\epsilon}$ and $\varphi^{\epsilon^{\prime}}$ are $G$-blow-Nash equivalent, they have the same quadratic part up to permutation of the variables $x_{1}, x_{4}, \ldots, x_{n}$ and, by [13] Proposition 3.14, $\epsilon=\epsilon^{\prime}$. Furthermore, we will show in corollary 8.3 below that the germs

$$
\varphi^{\epsilon,+}(x):=+x_{1}^{2}+x_{2}^{3}+\epsilon x_{3}^{4}+Q \text { and } \varphi^{\epsilon,-}(x):=-x_{1}^{2}+x_{2}^{3}+\epsilon x_{3}^{4}+Q^{\prime},
$$

where $\epsilon \in\{-1 ;+1\}$ and $+x_{1}^{2}+Q$ and $-x_{1}^{2}+Q^{\prime}$ are the same quadratic part up to permutation of the variables $x_{1}, x_{4}, \ldots, x_{n}$, are not $G$-blow-Nash equivalent.

If two germs $\omega^{\epsilon}$ and $\omega^{\epsilon^{\prime}}$ are $G$-blow-Nash equivalent, they also have the same quadratic part, up to permutation of the variables $x_{3}, \ldots, x_{n}$, and $\epsilon=\epsilon^{\prime}$ as well. 
If now two germs $\varphi^{\epsilon}$ and $\omega^{\epsilon^{\prime}}$ are $G$-blow-Nash equivalent, then $\epsilon=\epsilon^{\prime}$ and $\pm x_{1}^{2}+Q$ and $\pm x_{3}^{2}+Q^{\prime}$ are the same quadratic part up to permutation of all variables, so that we will intend to compare the germs

$$
\varphi(x)=x_{2}^{3}+\epsilon x_{3}^{4}+\eta x_{1}^{2}+Q \text { and } \omega(x)=x_{2}^{3}+\epsilon x_{1}^{4}+\eta^{\prime} x_{3}^{3}+Q^{\prime}
$$

where $\epsilon, \eta, \eta^{\prime} \in\{1,-1\}$ and $\eta x_{1}^{2}+Q=\eta^{\prime} x_{3}^{3}+Q^{\prime}$ up to permutation of all variables.

As in the previous two parts, we will consider the respective equivariant zeta functions of $\varphi$ and $\omega$, along with theorem 4.2 , to try to distinguish these invariant germs with respect to $G$-blow-Nash equivalence.

We begin with the computation of the first coefficients $\beta^{G}\left(A_{2}(\varphi)\right), \beta^{G}\left(A_{3}(\varphi)\right), \beta^{G}\left(A_{4}(\varphi)\right)$ of the naive equivariant zeta function of $\varphi$ (notice that the set $A_{1}(\varphi)$ is empty so that $\beta^{G}\left(A_{1}(\varphi)\right)=$ $0)$. Thanks to proposition 6.3 , we can focus on the quantities $\beta^{G}\left(A_{m}^{0}(\varphi)\right), m \leq 4$. The corresponding expressions for $\omega$ are similar, in this case equipping the set $Y_{p, q}$ with the trivial action of $G$.

Proposition 8.1. Write $\varphi=\varphi(x, z, y)=x^{3}+\epsilon z^{4}+Q_{p, q}(y)$. We have $\beta^{G}\left(A_{2}^{0}(\varphi)\right)=u^{4+p+q} \beta^{G}\left(Y_{p, q}\right)$, $\beta^{G}\left(A_{3}^{0}(\varphi)\right)=u^{2(p+q)+5} \beta^{G}\left(Y_{p, q} \backslash\{0\}\right)+\frac{u^{2(p+q)+6}}{u-1}$ and $\beta^{G}\left(A_{4}^{0}(\varphi)\right)=u^{3(p+q)+6} \beta^{G}\left(Y_{p, q} \backslash\{0\}\right)+$ $u^{2(p+q)+6} \beta^{G}(\{\varphi(0, z, y)=0\})$.

Proof. If $m \geq 1$, we write an $\operatorname{arc} \gamma$ of $\mathcal{L}_{m}$ as

$$
\begin{aligned}
\gamma(t) & =\left(a_{1} t+\cdots+a_{m} t^{m}, b_{1} t+\cdots+b_{m} t^{m}, c_{1}^{1} t+\cdots+c_{m}^{1} t^{m}, \ldots, c_{1}^{p+q} t+\cdots+c_{m}^{p+q} t^{m}\right) \\
& =\left(\begin{array}{c}
a_{1} \\
b_{1} \\
c_{1}^{1} \\
\vdots \\
c_{1}^{p+q}
\end{array}\right) t+\cdots+\left(\begin{array}{c}
a_{m} \\
b_{m} \\
c_{m}^{1} \\
\vdots \\
c_{m}^{p+q}
\end{array}\right) t^{m}=\left(\begin{array}{c}
a_{1} \\
b_{1} \\
c_{1}
\end{array}\right) t+\cdots+\left(\begin{array}{c}
a_{m} \\
b_{1} \\
c_{m}
\end{array}\right) t^{m}
\end{aligned}
$$

(the group $G$ acts only changing the sign of the coordinates $c_{i}^{1}$, resp. $c_{i}^{p+1}$, in the case $\mathrm{n}^{\circ} 1$, resp. $\left.\mathrm{n}^{\mathrm{O}} 2\right)$.

The set $A_{2}^{0}(\varphi)$ is described by the single equation $Q_{p, q}\left(c_{1}\right)=0$, the other variables remaining free. The set $A_{3}^{0}(\varphi)$ is defined by the system

$$
\left\{\begin{array}{l}
Q_{p, q}\left(c_{1}\right)=0, \\
a_{1}^{3}+\Phi_{p, q}\left(c_{1}, c_{2}\right)=0,
\end{array}\right.
$$

and, stratifying with the $G$-globally invariant subsets $\left\{c_{1}^{1}=\ldots=c_{1}^{i-1}=0, c_{1}^{i} \neq 0\right\}, i=1, \ldots, p$, and $\left\{c_{1}^{1}=\ldots=c_{1}^{p}=0\right\}=\left\{c_{1}=0\right\}$, we obtain

$$
\beta^{G}\left(A_{3}^{0}(\varphi)\right)=u^{6+2(p-1)+2 q+1} \beta^{G}\left(Y_{p, q} \backslash\{0\}\right)+\beta^{G}\left(A_{3}^{0}(\varphi) \cap\left\{c_{1}=0\right\}\right) .
$$

If $c_{1}=0$, then $a_{1}=0$ and the other variables are free, hence the desired expression. 
Finally, $A_{4}^{0}(\varphi)$ is described by the system of equations

$$
\left\{\begin{array}{l}
Q_{p, q}\left(c_{1}\right)=0 \\
a_{1}^{3}+\Phi_{p, q}\left(c_{1}, c_{2}\right)=0 \\
\epsilon b_{1}^{4}+3 a_{1}^{2} a_{2}+Q_{p, q}\left(c_{2}\right)+\Phi\left(c_{1}, c_{3}\right)=0
\end{array}\right.
$$

Equivariantly stratifying $A_{4}^{0}(\varphi)$ as we did for $A_{3}^{0}(\varphi)$, we get the equality

$$
\beta^{G}\left(A_{4}^{0}(\varphi)\right)=u^{8+3(p-1)+3 q+1} \beta^{G}\left(Y_{p, q} \backslash\{0\}\right)+\beta^{G}\left(A_{4}^{0}(\varphi) \cap\left\{c_{1}=0, a_{1}=0\right\}\right),
$$

the set $A_{4}^{0}(\varphi) \cap\left\{c_{1}=0, a_{1}=0\right\}$ being given by the equation $\epsilon b_{1}^{4}+Q_{p, q}\left(c_{2}\right)=0$.

With the same way of computation, we obtain the following expressions for the first terms of the equivariant zeta functions with signs of $\varphi$ :

Proposition 8.2. We have $\beta^{G}\left(A_{2}^{\xi}(\varphi)\right)=u^{4+p+q} \beta^{G}\left(Y_{p, q}^{\xi}\right), \beta^{G}\left(A_{3}^{\xi}(\varphi)\right)=u^{2(p+q)+5} \beta^{G}\left(Y_{p, q} \backslash\right.$ $\{0\})+\frac{u^{2(p+q)+6}}{u-1}$ and $\beta^{G}\left(A_{4}^{\xi}(\varphi)\right)=u^{3(p+q)+6} \beta^{G}\left(Y_{p, q} \backslash\{0\}\right)+u^{2(p+q)+6} \beta^{G}\left(\left\{\varphi\left(0, x_{3}, y\right)=\xi\right\}\right)$.

As we did in sections 6.1, 6.2 and 7.1, 7.2, we deduce the following distinctions:

Corollary 8.3. 1. The germs $\varphi^{\epsilon,+}$ and $\varphi^{\epsilon,-}$ are not G-blow-Nash equivalent.

2. If $p \leq q$ and $\eta=+1$ or $q \leq p$ and $\eta=-1$, then the germs $\varphi$ and $\omega$ are not $G$-blow-Nash equivalent.

3. If $p=q+1$ or $q=p+1$, then $\varphi$ and $\omega$ are not $G$-blow-Nash equivalent.

If $p>q+1$ and $\eta=+1$ or $q>p+1$ and $\eta=-1$, the respective quantities $\beta^{G}\left(Y_{p, q}\right)$ and $\beta^{G}\left(Y_{p, q}^{\xi}\right)$ are identical for $\varphi$ and $\omega$. Furthermore, notice that, equivariantly, $\left\{\varphi\left(0, x_{3}, y\right)=0\right\}=$ $\left\{f_{3}\left(x_{2}, y\right)=0\right\}$, resp. $\left\{\varphi\left(0, x_{3}, y\right)=\xi\right\}=\left\{f_{3}\left(x_{2}, y\right)=\xi\right\}$, and $\left\{\omega\left(0, x_{1}, y\right)=0\right\}=\left\{g_{2}\left(x_{1}, y\right)=\right.$ $0\}$, resp. $\left\{\omega\left(0, x_{1}, y\right)=\xi\right\}=\left\{g_{2}\left(x_{1}, y\right)=\xi\right\}$. Therefore, thanks to the computations of paragraph 6.2 , we can state the following :

Proposition 8.4. Suppose that $p>q+1$ and $\eta=+1$ or $q>p+1$ and $\eta=-1$.

1. For $m \leq 4, \beta^{G}\left(A_{m}(\varphi)\right)=\beta^{G}\left(A_{m}(\omega)\right)$.

2. For $m \leq 3, \beta^{G}\left(A_{m}^{\xi}(\varphi)\right)=\beta^{G}\left(A_{m}^{\xi}(\omega)\right)$.

3. - If $\eta=\epsilon$, then $\beta^{G}\left(A_{4}^{\xi}(\varphi)\right)=\beta^{G}\left(A_{4}^{\xi}(\omega)\right)$.

- If $\eta=+1$ and $\epsilon=-1$, then $\beta^{G}\left(A_{4}^{\xi}(\varphi)\right)=\beta^{G}\left(A_{4}^{\xi}(\omega)\right)$ if and only if the equivariant virtual Poincaré series of the algebraic subsets $\left\{-x_{3}^{4}+y^{2}+\sum_{i=1}^{K-1} y_{i}^{2}=\xi\right\} \subset$ $\mathbb{R}^{K+1}, K:=p-q$, equipped with the action of $G$ only changing the sign of $y$, and $\left\{-x_{1}^{4}+\sum_{i=1}^{K} z_{i}^{2}=\xi\right\} \subset \mathbb{R}^{K+1}$, equipped with the action of $G$ only changing the sign of $x_{1}$, are equal. 
- If $\eta=-1$ and $\epsilon=+1$, then we have $\beta^{G}\left(A_{4}^{\xi}(\varphi)\right)=\beta^{G}\left(A_{4}^{\xi}(\omega)\right)$ if and only if $\beta^{G}\left(\left\{x_{3}^{4}-y^{2}-\sum_{i=1}^{K-1} y_{i}^{2}=\xi\right\}\right)=\beta^{G}\left(\left\{x_{1}^{4}-\sum_{i=1}^{K} z_{i}^{2}=\xi\right\}\right)$.

For these cases, we have then to look at the other coefficients of the equivariant zeta functions of $\varphi$ and $\omega$. We begin by showing that, under this hypothesis $p>q+1, \eta=+1$ or $q>p+1, \eta=-1$, the respective naive equivariant zeta functions of $\varphi$ and $\omega$ are equal :

Proposition 8.5. If $p>q+1$ and $\eta=+1$ or $q>p+1$ and $\eta=-1$, then, for all $M>4$, we have $\beta^{G}\left(A_{M}(\varphi)\right)=\beta^{G}\left(A_{M}(\omega)\right)$.

Proof. Let $M$ be greater than 4 , we prove that $\beta^{G}\left(A_{M}^{0}(\varphi)\right)=\beta^{G}\left(A_{M}^{0}(\omega)\right)$. If we consider the system defining the two latter sets, the same computations as in proposition 8.1 provide an equal (under our current hypothesis) contribution of $\beta^{G}\left(Y_{p, q} \backslash\{0\}\right)$ and we are reduced to consider a system whose first condition is $a_{1}=0$ and next equation is (after a shift of indices) $\epsilon b_{1}^{4}+Q_{p, q}\left(c_{1}\right)=0$. This equation induces equal contributions for $\beta^{G}\left(A_{M}^{0}(\varphi)\right)$ and $\beta^{G}\left(A_{M}^{0}(\omega)\right)$ as well (recall that $\left\{\varphi\left(0, x_{3}, y\right)=0\right\}=\left\{f_{3}\left(x_{2}, y\right)=0\right\}$ and $\left\{\omega\left(0, x_{1}, y\right)=0\right\}=\left\{g_{2}\left(x_{1}, y\right)=0\right\}$ ).

We then stratify with respect to the coordinates of $c_{1}$ as we did in the proofs of propositions 6.20 and 7.11 , and we obtain the further condition $b_{1}=0$. The first subsequent equations become, if $M \geq 8$,

$$
\left\{\begin{array}{l}
a_{2}^{3}+Q_{p, q}\left(c_{1}\right)=0 \\
3 a_{2}^{2} a_{3}+\Phi_{p, q}\left(c_{1}, c_{2}\right)=0 \\
\epsilon b_{2}^{4}+3 a_{2} a_{3}^{2}+3 a_{2}^{2} a_{4}+Q_{p, q}\left(c_{2}\right)+\Phi\left(c_{1}, c_{3}\right)=0
\end{array}\right.
$$

Another stratification with respect to the vector $c_{1}$ provides an equal (by lemma 7.12) contribution of $\beta^{G}\left(\left\{\varphi\left(x_{2}, 0, y\right)=0\right\}\right)=\beta^{G}\left(\left\{h_{4}\left(0, x_{3}, y\right)=0\right\}\right)$, respectively $\beta^{G}\left(\left\{\omega\left(x_{2}, 0, y\right)=\right.\right.$ $0\})=\beta^{G}\left(\left\{r_{3}\left(0, x_{2}, y\right)=0\right\}\right)$, and the condition $a_{2}=0$.

Carrying on with the computation, we obtain the equivalence $\beta^{G}\left(A_{M}^{0}(\varphi)\right)=\beta^{G}\left(A_{M}^{0}(\omega)\right)$ if and only if $\beta^{G}(\{\varphi=0\})=\beta^{G}(\{\omega=0\})$, from the equations of the form $\epsilon b_{j}^{4}+a_{j^{\prime}}^{3}+Q_{p, q}\left(c_{1}\right)=0$ with $4 j=3 j^{\prime}$. We prove in lemma 8.6 below that $\beta^{G}(\{\varphi=0\})=\beta^{G}(\{\omega=0\})$.

Lemma 8.6. Suppose that $p>q+1$ and $\eta=+1$ or $q>p+1$ and $\eta=-1$. Then

$$
\beta^{G}(\{\varphi=0\})=\beta^{G}(\{\omega=0\}) .
$$

Proof. Suppose that $p>q+1$ and $\eta=+1$. Considering an equivariant resolution of singularities of the $G$-algebraic set $\{\omega=0\}$, we will compare the quantities $\beta^{G}(\{\omega=0\})$ and $\beta^{G}(\{\varphi=0\})$.

Write $\omega(x)=x^{3}+\epsilon z^{4}+Q_{p, q}(y)$ (the group $G$ acts via the involution $(x, z, y) \mapsto(x,-z, y)$ ). Using an equivariant change of coordinates as in the proof of proposition 5.1, we can assume $q=0$. We then equivariantly blow-up the $G$-algebraic set $\{\omega=0\}$ at the origin of $\mathbb{R}^{n}$ :

- in the chart $x=u, z=u v, y_{i}=u w_{i}$, with $G$-action $\left(u, v, w_{i}\right) \mapsto\left(u,-v, w_{i}\right)$, the equation of the blown-up variety is

$$
u^{2}\left[u+\epsilon u^{2} v^{4}+Q_{p, q}(w)\right]=0
$$


- in the chart $x=v u, z=v, y_{i}=v w_{i}$, with $G$-action $\left(u, v, w_{i}\right) \mapsto\left(-u,-v,-w_{i}\right)$, it is

$$
v^{2}\left[v u^{3}+\epsilon v^{2}+Q_{p, q}(w)\right]=0,
$$

- in the respective charts $x=w_{j} u, z=w_{j} v, y_{j}=w_{j}, y_{i}=w_{j} w_{i}$ for $i \neq j$, with $G$-action $\left(u, v, w_{i}\right) \mapsto\left(u, v, w_{i}\right)$, it is

$$
w_{j}^{2}\left[w_{j} u^{3}+\epsilon w_{j}^{2} v^{4}+1+Q(\widehat{w})\right]=0 .
$$

The set of points of the strict transform of $\{\omega=0\}$ which are in the first chart but not in the second one is given by $v=0, u+Q_{p, q}(w)=0$, therefore it is equivariantly isomorphic to an affine space : the respective induced contributions for $\beta^{G}(\{\omega=0\})$ and $\beta^{G}(\{\varphi=0\})$ are equal. Now, the set of points of the strict transform which are in one of the last charts but not in the second and the first ones is given by $v=0, u=0,1+Q(\widehat{w})=0$ : it is the empty set $(q=0)$.

Furthermore, notice that the intersection of the strict transform of $\{\omega=0\}$ with the exceptional divisor is a circle with a nonempty fixed point set.

Consequently, we are reduced to consider the equivariant virtual Poincaré series of the algebraic set of $\mathbb{R}^{n}$ defined by the equation $z x^{3}+\epsilon z^{2}+Q_{p, q}(y)=0, G$ acting via $(x, z, y) \mapsto$ $(-x,-z,-y)$ (for $\varphi$, the involution would have been $\left.\left(x, z, y_{1}, y_{i}\right) \mapsto\left(x, z,-y_{1}, y_{i}\right)\right)$. We equivariantly blow-up this $G$-algebraic set at the origin of $\mathbb{R}^{n}$ as well :

- in the chart $x=u, z=u v, y_{i}=u w_{i}$, with $G$-action $\left(u, v, w_{i}\right) \mapsto\left(-u, v, w_{i}\right)$, the equation of the blown-up variety is

$$
u^{2}\left[v u^{2}+\epsilon v^{2}+Q_{p, q}(w)\right]=0,
$$

- in the chart $x=v u, z=v, y_{i}=v w_{i}$, with $G$-action $\left(u, v, w_{i}\right) \mapsto\left(u,-v, w_{i}\right)$, it is

$$
v^{2}\left[v^{2} u^{3}+\epsilon+Q_{p, q}(w)\right]=0,
$$

- in the respective charts $x=w_{j} u, z=w_{j} v, y_{j}=w_{j}, y_{i}=w_{j} w_{i}$ for $i \neq j$, with $G$-action $\left(u, v, w_{j}, w_{i}\right) \mapsto\left(u, v,-w_{j}, w_{i}\right)$, it is

$$
w_{j}^{2}\left[v w_{j}^{2} u^{3}+\epsilon v^{2}+1+Q(\widehat{w})\right]=0 .
$$

The set of points of the strict transform which are in the second chart but not in the first one is given by $u=0, \epsilon+Q_{p, q}(w)=0$ : it is the cartesian product of an affine line and the set $Y_{p, q}^{-\epsilon}$, and therefore it induces an equal contribution for $\beta^{G}(\{\omega=0\})$ and $\beta^{G}(\{\varphi=0\})$ under our current hypothesis. As for the set of points of the strict transform which are in one of the last charts but not in the first and the second ones, it is given by $u=0, v=0,1+Q(\widehat{w})=0$, thus it is empty.

On the other hand, the intersection of the strict transform with the exceptional divisor provides equal contributions for $\beta^{G}(\{\omega=0\})$ and $\beta^{G}(\{\varphi=0\})$ as well. 
As a consequence, we can focus on the equation $z x^{2}+\epsilon z^{2}+Q_{p, q}(y)=0$ in $\mathbb{R}^{n}$, the group $G$ acting via $(x, z, y) \mapsto(-x, z, y)$ for $\omega$ (respectively via $\left(x, z, y_{1}, y_{i}\right) \mapsto\left(x, z,-y_{1}, y_{i}\right)$ for $\varphi$ ). We equivariantly blow-up once again :

- in the chart $x=u, z=u v, y_{i}=u w_{i}$, with $G$-action $\left(u, v, w_{i}\right) \mapsto\left(-u,-v,-w_{i}\right)$, the equation of the blown-up variety is

$$
u^{2}\left[u v+\epsilon v^{2}+Q_{p, q}(w)\right]=0,
$$

- in the chart $x=v u, z=v, y_{i}=v w_{i}$, with $G$-action $\left(u, v, w_{i}\right) \mapsto\left(-u, v, w_{i}\right)$, it is

$$
v^{2}\left[v u^{2}+\epsilon+Q_{p, q}(w)\right]=0
$$

- in the respective charts $x=w_{j} u, z=w_{j} v, y_{j}=w_{j}, y_{i}=w_{j} w_{i}$ for $i \neq j$, with $G$-action $\left(u, v, w_{i}\right) \mapsto\left(-u, v, w_{i}\right)$, it is

$$
w_{j}^{2}\left[v w_{j} u^{2}+\epsilon v^{2}+1+Q(\widehat{w})\right]=0 .
$$

By similar arguments as above, we are reduced to consider the equation $u v+\epsilon v^{2}+Q_{p, q}(w)=$ 0 . We can then stratify with respect to $v$ and show that the induced respective contributions for $\beta^{G}(\{\varphi=0\})$ and $\beta^{G}(\{\omega=0\})$ are also the same. This finally proves the equality $\beta^{G}(\{\varphi=$ $0\})=\beta^{G}(\{\omega=0\})$.

Similarly to what we did in the proofs of propositions 6.21 and 7.13 , we can adapt the proof of proposition 8.5 in order to state a sufficient and necessary condition for the equality of the respective equivariant zeta functions with signs of $\varphi$ and $\omega$ to be true :

Proposition 8.7. 1. Suppose $p>q+1$ and $\eta=+1$. Then $Z_{\varphi}^{G, \xi}(u, T)=Z_{\omega}^{G, \xi}(u, T)$ if and only if we have the equalities

- $\beta^{G}\left(\left\{x_{2}^{3}+y^{2}+\sum_{i=1}^{K-1} y_{i}^{2}=\xi\right\}\right)=\beta^{G}\left(\left\{x_{2}^{3}+\sum_{i=1}^{K} z_{i}^{2}=\xi\right\}\right)$,

- $\beta^{G}\left(\left\{\epsilon x_{3}^{4}+y^{2}+\sum_{i=1}^{K-1} y_{i}^{2}=\xi\right\}\right)=\beta^{G}\left(\left\{\epsilon x_{1}^{4}+\sum_{i=1}^{K} z_{i}^{2}=\xi\right\}\right)$,

- and $\beta^{G}\left(\left\{x_{2}^{3}+\epsilon x_{3}^{4}+y^{2}+\sum_{i=1}^{K-1} y_{i}^{2}=\xi\right\}\right)=\beta^{G}\left(\left\{x_{2}^{3}+\epsilon x_{1}^{4}+\sum_{i=1}^{K} z_{i}^{2}=\xi\right\}\right)$,

where, in the left members of the equalities, the considered sets are algebraic subsets of $\mathbb{R}^{K+2}$ equipped with the action of $G$ only changing the sign of $y$, and, in the right members, the sets are subsets of $\mathbb{R}^{K+2}$ equipped with the action of $G$ only changing the sign of $x_{1}$.

2. Suppose $q>p+1$ and $\eta=-1$. Then $Z_{\varphi}^{G, \xi}(u, T)=Z_{\omega}^{G, \xi}(u, T)$ if and only if we have the equalities

- $\beta^{G}\left(\left\{x_{2}^{3}-y^{2}-\sum_{i=1}^{K-1} y_{i}^{2}=\xi\right\}\right)=\beta^{G}\left(\left\{x_{2}^{3}-\sum_{i=1}^{K} z_{i}^{2}=\xi\right\}\right)$,

- $\beta^{G}\left(\left\{\epsilon x_{3}^{4}-y^{2}-\sum_{i=1}^{K-1} y_{i}^{2}=\xi\right\}\right)=\beta^{G}\left(\left\{\epsilon x_{1}^{4}-\sum_{i=1}^{K} z_{i}^{2}=\xi\right\}\right)$,

- and $\beta^{G}\left(\left\{x_{2}^{3}+\epsilon x_{3}^{4}-y^{2}-\sum_{i=1}^{K-1} y_{i}^{2}=\xi\right\}\right)=\beta^{G}\left(\left\{x_{2}^{3}+\epsilon x_{1}^{4}-\sum_{i=1}^{K} z_{i}^{2}=\xi\right\}\right)$. 
Remark 8.8. As we showed in the proof of proposition 6.17 , we have $\beta^{G}\left(\left\{+x_{3}^{4}+y^{2}+\sum_{i=1}^{K-1} y_{i}^{2}=\right.\right.$ $\xi\})=\beta^{G}\left(\left\{+x_{1}^{4}+\sum_{i=1}^{K} z_{i}^{2}=\xi\right\}\right)$ and $\beta^{G}\left(\left\{-x_{3}^{4}-y^{2}-\sum_{i=1}^{K-1} y_{i}^{2}=\xi\right\}\right)=\beta^{G}\left(\left\{-x_{1}^{4}-\sum_{i=1}^{K} z_{i}^{2}=\xi\right\}\right)$ for $\xi= \pm 1$.

We finally gather the results of this section in one theorem :

Theorem 8.9. Suppose that the invariant germs

$$
\varphi(x)=x_{2}^{3}+\epsilon x_{3}^{4}+\eta x_{1}^{2}+Q \text { and } \omega(x)=x_{2}^{3}+\epsilon x_{1}^{4}+\eta^{\prime} x_{3}^{3}+Q^{\prime}
$$

have, up to permutation of all variables, the same quadratic part, with $p$ signs + and $q$ signs - .

1. If

- $p \leq q, \eta=+1$ or $q \leq p, \eta=-1$,

- $p=q+1$ or $q=p+1$,

then $\varphi$ and $\omega$ are not $G$-blow-Nash equivalent.

2. - If $p>q+1, \eta=+1$, then $Z_{\varphi}^{G}(u, T)=Z_{\omega}^{G}(u, T)$. Furthermore, $Z_{\varphi}^{G, \xi}(u, T)=$ $Z_{\omega}^{G, \xi}(u, T)$ if and only if $\beta^{G}\left(\left\{x_{2}^{3}+y^{2}+\sum_{i=1}^{K-1} y_{i}^{2}=\xi\right\}\right)=\beta^{G}\left(\left\{x_{2}^{3}+\sum_{i=1}^{K} z_{i}^{2}=\xi\right\}\right)$, $\beta^{G}\left(\left\{\epsilon x_{3}^{4}+y^{2}+\sum_{i=1}^{K-1} y_{i}^{2}=\xi\right\}\right)=\beta^{G}\left(\left\{\epsilon x_{1}^{4}+\sum_{i=1}^{K} z_{i}^{2}=\xi\right\}\right)$ and $\beta^{G}\left(\left\{x_{2}^{3}+\epsilon x_{3}^{4}+y^{2}+\right.\right.$ $\left.\left.\sum_{i=1}^{K-1} y_{i}^{2}=\xi\right\}\right)=\beta^{G}\left(\left\{x_{2}^{3}+\epsilon x_{1}^{4}+\sum_{i=1}^{K} z_{i}^{2}=\xi\right\}\right)$.

- If $q>p+1, \eta=-1$, then $Z_{\varphi}^{G}(u, T)=Z_{\omega}^{G}(u, T)$. Furthermore, $Z_{\varphi}^{G, \xi}(u, T)=$ $Z_{\omega}^{G, \xi}(u, T)$ if and only if $\beta^{G}\left(\left\{x_{2}^{3}-y^{2}-\sum_{i=1}^{K-1} y_{i}^{2}=\xi\right\}\right)=\beta^{G}\left(\left\{x_{2}^{3}-\sum_{i=1}^{K} z_{i}^{2}=\xi\right\}\right)$, $\beta^{G}\left(\left\{\epsilon x_{3}^{4}-y^{2}-\sum_{i=1}^{K-1} y_{i}^{2}=\xi\right\}\right)=\beta^{G}\left(\left\{\epsilon x_{1}^{4}-\sum_{i=1}^{K} z_{i}^{2}=\xi\right\}\right)$ and $\beta^{G}\left(\left\{x_{2}^{3}+\epsilon x_{3}^{4}-y^{2}-\right.\right.$ $\left.\left.\sum_{i=1}^{K-1} y_{i}^{2}=\xi\right\}\right)=\beta^{G}\left(\left\{x_{2}^{3}+\epsilon x_{1}^{4}-\sum_{i=1}^{K} z_{i}^{2}=\xi\right\}\right)$.

Remark 8.10. Forgetting the $G$-action, the respective virtual Poincaré polynomials of the algebraic subsets $\left\{x^{3}+\epsilon z^{4}+\sum_{i=1}^{K} y_{i}^{2}=\xi\right\}$ and $\left\{x^{3}+\epsilon z^{4}-\sum_{i=1}^{K} y_{i}^{2}=\xi\right\}, \epsilon= \pm 1, \xi= \pm 1$, of $\mathbb{R}^{K+1}$ can be computed using the invariance of the virtual Poincaré polynomial under bijection with $\mathcal{A S}$ graph (see also remarks 6.19 and 7.15). If the equivariant virtual Poincaré series was shown to be an invariant under equivariant $\mathcal{A S}$ bijection, it should be possible to compute the above considered quantities.

\section{References}

[1] V. I. Arnold, Critical points of function on a manifold with boundary, the simple Lie groups $B_{k}, C_{k}, F_{4}$, and singularities of evolutes, Uspekhi Mat. Nauk 33 (1978), no. 5, 91-105; English transl. Russian Math. Surveys 33 (1978), no. 5, 99-116.

[2] V. I. Arnold, S. M. Gusein-Zade, A. N. Varchenko, Singularities of Differentiable Maps, Volumes 1 and 2, Birkhäuser, Boston, 1985.

[3] M. Artin, Algebraic approximation of structures over complete local rings, Publ. Math. IHES, 36 (1969), 23-58. 
[4] E. Bierstone, P. Milman, Invariant solutions of analytic equations, Enseign. Math. (2), 25 (1979), 115-130.

[5] J. Bochnak, M. Coste, M.-F. Roy, Real Algebraic Geometry, Springer-Verlag, Berlin, 1998.

[6] J.-B. Campesato, On a motivic invariant of the arc-analytic equivalence, to appear in Annales de l'Institut Fourier.

[7] M. Coste, J. Ruiz, M. Shiota, Approximation in compact Nash manifolds, Amer. J. Math., 117, no. 4 (1995), 905-927.

[8] J. Denef, F. Loeser, Germs of arcs on singular algebraic varieties and motivic integration, Invent. math. 135 (1999), 201-232.

[9] G. Fichou, Motivic invariants of Arc-symmetric sets and Blow-Nash equivalence, Compositio Math. 141 (2005) 655-688.

[10] G. Fichou, Zeta functions and Blow-Nash equivalence, Annales Polonici Math. 87 (2005) 111-125.

[11] G. Fichou, The corank and index are blow-Nash invariants, Kodai Math. J. 29 (2006) $31-40$.

[12] G. Fichou, Toward a classification of blow-Nash types, (RIMS, Kyoto 2006), RIMS Kôkyûroku 1540, 145-151, 2007.

[13] G. Fichou, Blow-Nash types of simple singularities, J. Math. Soc. Japan 60, no. 2 (2008), 445-470.

[14] G. Fichou, Equivariant virtual Betti numbers, Ann. de l'Inst. Fourier, 58, no. 1 (2008), $1-27$.

[15] T. Fukui, Seeking invariants for blow-analytic equivalence, Compositio Math. 105 (1997), 95-107.

[16] V. V. Goryunov, Bifurcations with symmetries, in Singularity Theory and Some Problems of Functional Analysis, S.G. Gindikin ed., AMS Translations, ser. 2, vol. 153, 1992, 93-108.

[17] S. Koike, A. Parusiński, Motivic-type invariants of blow-analytic equivalence, Ann. Inst. Fourier 53 (2003), 2061-2104.

[18] T.-C. Kuo, On classification of real singularities, Invent. Math. 82 (1985), 257-262.

[19] K. Kurdyka, Ensembles semi-algébriques symétriques par arcs, Math. Ann. 281 (1988), 445-462.

[20] K. Kurdyka, A. Parusiński, Arc-symmetric sets and arc-analytic mappings, Panoramas et Synthèses 24, Soc. Math. France (2007), 33-67. 
[21] C. McCrory, A. Parusiński, Virtual Betti numbers of real algebraic varieties, C. R. Math. Acad. Sci. Paris 336 (2003), no. 9, 763-768.

[22] C. McCrory, A. Parusiński, The weight filtration for real algebraic varieties, in Topology of Stratified Spaces, G. Friedman et al. eds., MSRI Publ. 58, Cambridge University Press, New York 2011, 121-160.

[23] F. Priziac, Equivariant zeta functions for invariant Nash germs, Nagoya Mathematical Journal, 2016, doi: 10.1017/nmj.2016.12

[24] G. Rond, Local zero estimates and effective division in rings of algebraic power series, Journal fr die reine und angewandte Mathematik (Crelles Journal), 2015.

[25] G. Rond, Artin Approximaton, preprint.

[26] J. van Hamel, Algebraic cycles and topology of real algebraic varieties, CWI Tract 129, Stichting Mathematisch Centrum, Centrum voor Wiskunde en Informatica, Amsterdam, 1997.

Fabien PRIZIAC

Institut de Mathématiques de Marseille (UMR 7373 du CNRS)

Aix-Marseille Université

39, rue Frédéric Joliot-Curie

13453 Marseille Cedex 13

FRANCE

fabien.priziac@univ-amu.fr 